\title{
WestVirginiaUniversity
}

THE RESEARCH REPOSITORY @ WVU

Graduate Theses, Dissertations, and Problem Reports

2015

\section{Avoidance of Token-Loss by Pigeons}

James E. Cook

Follow this and additional works at: https://researchrepository.wvu.edu/etd

\section{Recommended Citation}

Cook, James E., "Avoidance of Token-Loss by Pigeons" (2015). Graduate Theses, Dissertations, and Problem Reports. 7072.

https://researchrepository.wvu.edu/etd/7072

This Dissertation is protected by copyright and/or related rights. It has been brought to you by the The Research Repository @ WVU with permission from the rights-holder(s). You are free to use this Dissertation in any way that is permitted by the copyright and related rights legislation that applies to your use. For other uses you must obtain permission from the rights-holder(s) directly, unless additional rights are indicated by a Creative Commons license in the record and/ or on the work itself. This Dissertation has been accepted for inclusion in WVU Graduate Theses, Dissertations, and Problem Reports collection by an authorized administrator of The Research Repository @ WVU.

For more information, please contact researchrepository@mail.wvu.edu. 


\title{
Avoidance of Token-Loss by Pigeons
}

\author{
James E. Cook
}

\author{
Dissertation submitted \\ to the Eberly College of Arts and Sciences at West Virginia University \\ in partial fulfillment of the requirements for the degree of \\ Doctor of Philosophy in \\ Psychology
}

\author{
Kennon A. Lattal, Ph.D., Chair \\ Claire C. St. Peter, Ph.D., BCBA-D \\ Michael Perone, Ph.D. \\ Steven G. Kinsey, Ph.D. \\ Daniel E. Hursh, Ph.D., BCBA-D \\ Department of Psychology
}

\section{Morgantown, West Virginia}

2015

Keywords: token; avoidance; conditioned reinforcement; negative reinforcement; pigeon; variable-cycle schedule Copyright 2015 James E. Cook 


\section{ABSTRACT \\ Avoidance of Token-Loss by Pigeons}

\section{James E. Cook}

The response-dependent delivery and removal of tokens can reinforce and punish the responding of humans and nonhuman animals. The responding of humans can be maintained by the avoidance of token-losses, but this has not been demonstrated with nonhuman animals. Across three experiments, it was demonstrated that the responding of pigeons could be maintained in a token-loss avoidance arrangement. Tokens were delivered response-independently at the beginning of a token-loss avoidance period. Tokens were scheduled to be removed on a variablecycle schedule. Responding canceled scheduled token-losses. Those tokens that remained were exchangeable for food during programmed exchange periods. Several parameters of the tokenloss avoidance arrangement were manipulated to assess their effects on responding. In Experiment 1, responding was a function of the frequency of scheduled token-losses. In Experiment 2, responding was a function of the frequency of exchange periods. In Experiment 3, no consistent changes in responding occurred whether a single token produced multiple reinforcers or multiple tokens were required to produce a single reinforcer. Across all experiments, local response rates were an inverse function of the number of tokens present. The similarity of these results to those obtained in previous experiments, the possible functions of tokens in the token-loss avoidance arrangement, and the implications of the methods used in the present series of experiments are discussed. 


\section{Acknowledgements}

I would like to thank Andy Lattal, Claire St. Peter, Mike Perone, Steve Kinsey, and Dan Hursh for serving on my committee, for their helpful comments on the design and implications of this work, and for their patience along the way. I would like to thank Ezra Hall, Stephanie Kincaid, Tyler Nighbor, and Sean Brown for their help in running these experiments and the many conversations about this and other projects. I would like to thank Donna and Dennis Cook for supporting and teaching me across my 25 years of education. Lastly, I am especially grateful to Andy Lattal for advising me on this project and across my graduate career, guiding me towards being a better researcher and behavior analyst, and for letting me drill holes in his operant chamber. 
Table of Contents

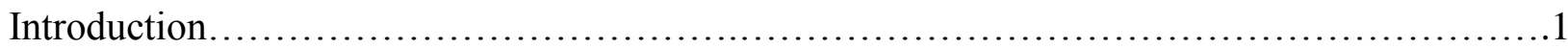

Literature Review..........................................................

Functions of Tokens...................................................

Conditioned Reinforcers........................................2

Pavlovian Processes..............................................

Generalized Conditioned Reinforcers..............................4

Discriminative Stimuli..........................................

Response cost............................................10

Parameters of Token Schedules of Reinforcement..........................12

Negative Reinforcement in Token Economies.............................17

Theoretical Benefits of Tokens.........................................18

Statement of the Problem................................................... 21

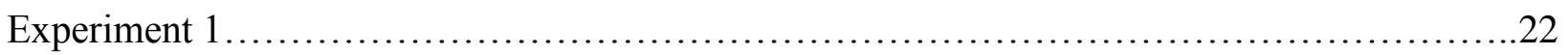

Method.................................................................. 23

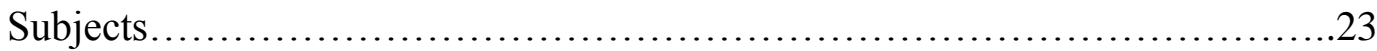

Apparatus.......................................................24

Procedure............................................................24

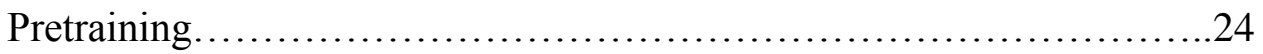

Effects of VC Schedules of Token-Loss Avoidance....................27

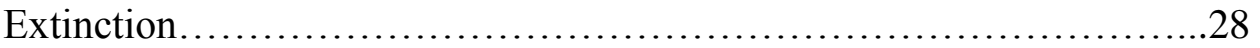

Results and Discussion..................................................28

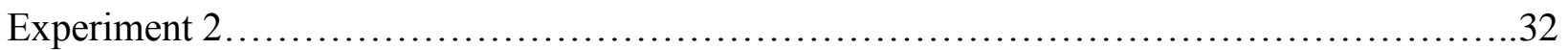


Subjects and Apparatus............................................................... 33

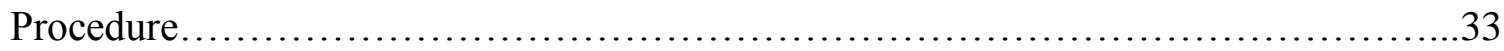

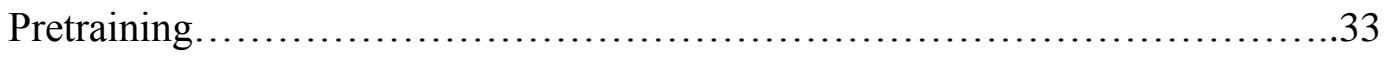

Effects of Exchange Period Frequency.....................................33

Results and Discussion....................................................... 34

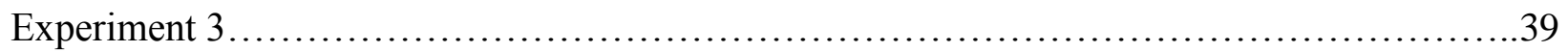

Subjects and Apparatus......................................................... 40

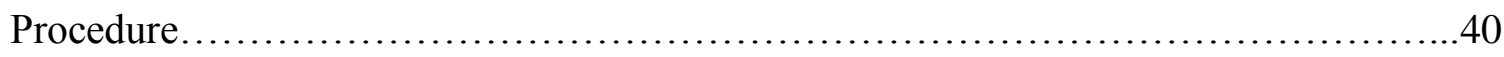

Results and Discussion..........................................................

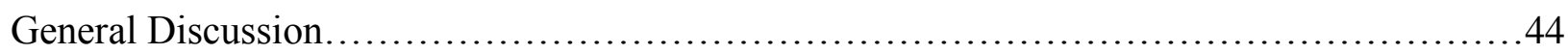

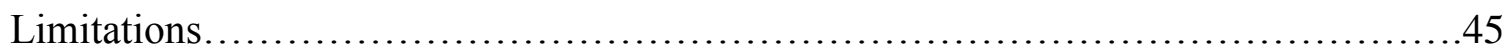

Functions of Tokens in the Current Token-Loss Avoidance Arrangement................47

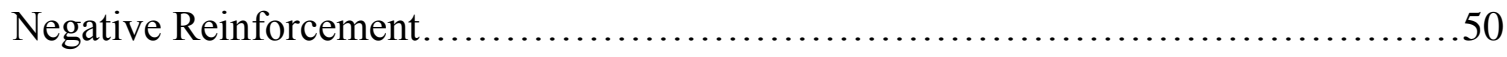

Basic Research/Theoretical Implications............................................51

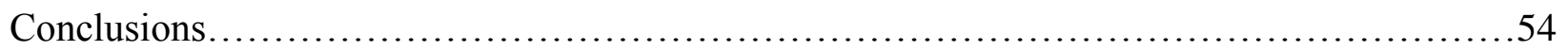

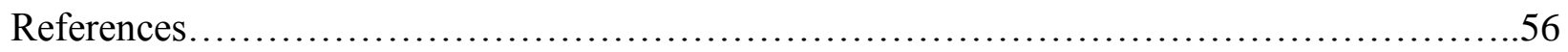

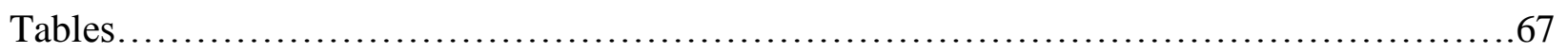

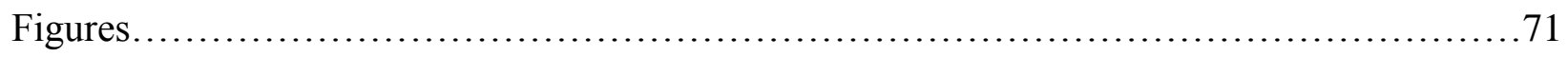

Appendix A: Reinstatement of Responding in a Token-Loss Avoidance Arrangement...........91

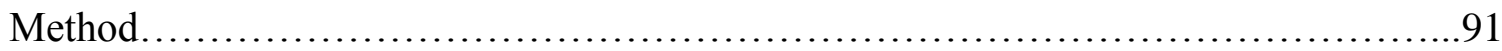

Extinction...........................................................91

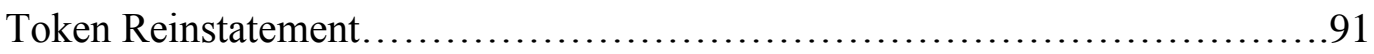

Token+Food Reinstatement............................................. 92 
$\mathrm{VC} 10 \mathrm{~s}$

Results and Discussion...................................................92

References................................................................... 95

Appendix B: Responding on a Multiple Schedule of Token-Loss Avoidance and Token-

Production................................................................. 101

Method.................................................................... 101

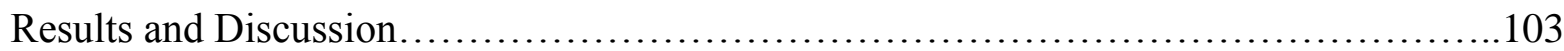

References................................................................. 105 
List of Tables

Table 1: Order of Conditions and Number of Sessions in Experiment 1.....................67

Table 2: Order of Conditions and Number of Sessions in Experiment 2...................68

Table 3: Order of Conditions and Number of Sessions in Experiment 3................... 70

Table B1: Average Frequency of Exchange Periods and Food Deliveries and the Percentage of Available Tokens Exchanged in the Avoidance and Production Components.................107 


\section{List of Figures}

Figure 1: Diagram of the first phase of pretraining .....................................

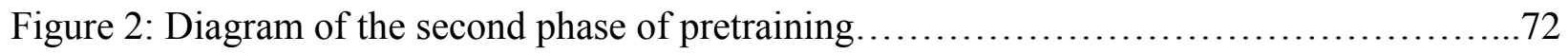

Figure 3: Diagram of the general procedure across the present series of experiments...........73

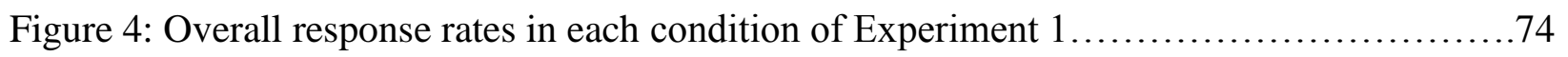

Figure 5: Local responding across tokens present in Experiment $1 \ldots \ldots \ldots \ldots \ldots \ldots \ldots \ldots \ldots \ldots \ldots$

Figure 6: Aggregate local responding across tokens present in Experiment $1 \ldots \ldots \ldots \ldots \ldots \ldots . . . \ldots 76$

Figure 7: Percent of scheduled token-losses canceled in each condition of Experiment 1.......77

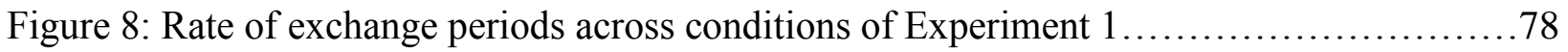

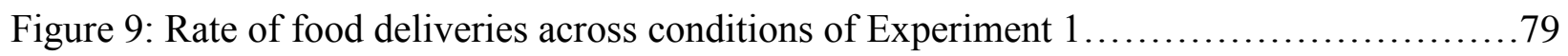

Figure 10: Overall response rates across conditions of Experiment $2 \ldots \ldots \ldots \ldots \ldots \ldots \ldots \ldots \ldots \ldots$

Figure 11: Local responding across tokens present in Experiment $2 \ldots \ldots \ldots \ldots \ldots \ldots \ldots \ldots \ldots . \ldots . \ldots \ldots$

Figure 12: Aggregate local responding across tokens present in Experiment 2..............82

Figure 13: Percent of scheduled token-losses canceled across conditions in Experiment 2......83

Figure 14: Rate of exchange periods across conditions of Experiment $2 \ldots \ldots \ldots \ldots \ldots \ldots \ldots \ldots$

Figure 15: Rate of food deliveries across conditions of Experiment $2 \ldots \ldots \ldots \ldots \ldots \ldots \ldots \ldots \ldots \ldots$

Figure 16: Overall response rates in each condition of Experiment $3 \ldots \ldots \ldots \ldots \ldots \ldots \ldots \ldots \ldots . \ldots . \ldots . \ldots$

Figure 17: Local responding across tokens present in Experiment $3 \ldots \ldots \ldots \ldots \ldots \ldots \ldots \ldots \ldots \ldots$

Figure 18: Percent of scheduled token-losses canceled across conditions in Experiment $3 \ldots . . .88$

Figure 19: Rate of exchange periods across conditions of Experiment $3 \ldots \ldots \ldots \ldots \ldots \ldots \ldots . \ldots . \ldots . \ldots$

Figure 20: Rate of food deliveries and token exchanges across conditions of Experiment 3.....90

Figure A1: Overall response rates across conditions in Experiment A......................96

Figure A2: Local response rates across tokens present in Experiment A.....................97 
Figure A3: Percent of scheduled token-losses across conditions in Experiment A..............98

Figure A4: Rate of exchange periods across conditions of Experiment A.....................99

Figure A5: Rate of token exchanges across conditions of Experiment A....................100

Figure B1: Overall response rates in the avoid and production components.................108

Figure B2: Local response rates in the avoid and production components across tokens present in

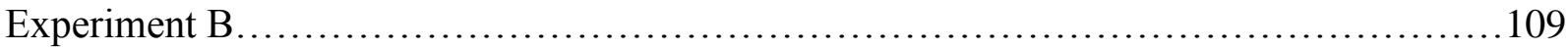


Avoidance of Token-Loss by Pigeons

When a reinforcer is delivered, it often is not delivered in isolation. When the hopper is raised for a pigeon, a light may turn on. When a pellet is dispensed for a rat, a tone may sound. When a client receives a toy for appropriate behavior, the therapist may also deliver praise. Through Pavlovian and operant processes, stimuli repeatedly and reliably paired with reinforcer delivery can acquire eliciting, discriminative, and reinforcing properties of their own (Williams, 1994). One use of these processes that has been useful in research and practice has been the development of the token economy (for reviews, see Dickerson, Tehhula, \& Green-Paden, 2005; Hackenberg, 2009; Kazdin, 1982; Kazdin \& Bootzin, 1972; Maggin, Chafouleas, Goddard, \& Johnson, 2011; Matson \& Boisjoli, 2009)

A token is an object or symbol that is exchangeable for goods or services. Tokens may be manipulable objects (e.g. coins, poker chips, stickers, etc.) or other stimuli (e.g., LED lights, points, check marks). Tokens are usually conceptualized as conditioned reinforcers that acquire their reinforcing function through repeated exchanges for unconditioned reinforcers (e.g., food) or established conditioned reinforcers (e.g., money). Tokens have a long history of being used in the laboratory as reinforcers for both humans (Byron, Himoff, Catania, \& Sagvolden, 1977; Critchfield, Paletz, Macaleese, \& Newland, 2003; King \& Logue, 1990; Magoon \& Critchfield, 2008; Silberberg et al., 2008; Weiner, 1962, 1963, 1969a, b) and nonhuman animals (Bullock \& Hackenberg, 2006, 2015; Cowles, 1937; Hackenberg, 2009; Kelleher, 1956, 1958; Malagodi, 1967a, b, c; Webbe \& Malagodi, 1978; Wolfe, 1936). Token schedules of reinforcement also underlie both monetary systems and critical components of behavior-management programs across numerous applied settings (e.g., Ayllon, \& Azrin, 1968; Conyers et al., 2004; DeLeon, 
Bullock, \& Catania, 2013; Fox, Hopkins \& Anger, 1987; Kahng, Boscoe, \& Byrne, 2003;

Kazdin, 1982; Kazdin \& Bootzin, 1972; Phillips, 1968).

In token economies, behavior is generally reinforced and maintained by the responsedependent production of tokens. With a few exceptions (e.g., Donaldson, DeLeon, Fisher, \& Kahng, 2014; Iwata \& Bailey, 1974; Magoon \& Critchfield, 2008), token economies have been studied almost exclusively in the context of their production following responses. The present series of experiments extends the analysis of token reinforcement to an avoidance (i.e., negative reinforcement) procedure with nonhuman animals.

\section{Literature Review}

\section{Functions of Tokens}

Conditioned reinforcers. Research on responding maintained by token delivery has a long history dating back to work by Wolfe (1936) and Cowles (1937). They conducted a series of experiments aimed at assessing the conditioned reinforcing effects of tokens. In their experiments, chimpanzees were trained to deposit poker chips into an apparatus that dispensed a variety of primary (or backup) reinforcers. Initially, Wolfe (1936) trained chimpanzees to discriminate between white tokens that were exchangeable for food and similar brass tokens that were not exchangeable for food. After this discrimination was trained, responding maintained by the delivery of food was compared to responding maintained by the delivery of tokens immediately exchangeable for food in a variety of conditions. For most of the chimpanzees, there was little difference between responding maintained by food delivery and responding maintained by token delivery.

Cowles (1937) used the same subjects in a follow-up to Wolfe's (1936) work. In a couple of Cowles's experiments, correct responding in a position discrimination task was reinforced 
immediately with food or with tokens exchangeable for food at the end of the session. The discrimination was acquired in both conditions. In these experiments, token delivery was a reinforcer for correct responding, but performance improved more rapidly when correct responses were immediately reinforced with food.

In subsequent experiments, Cowles (1937) demonstrated that the reinforcing functions of tokens were the result of their pairing with food. Chimpanzees responded in a delayed match-tosample task. In different conditions, correct responses resulted in the delivery of one of two types of tokens. Opportunities to exchange these tokens were delayed until the end of each session. Tokens were exchanged for either food (food-paired tokens) or nothing (nonfood-paired tokens). Accuracy improved in both conditions, but was higher when responding produced food-paired tokens. In a follow-up experiment, correct responding resulted in the delivery of food, foodpaired tokens, or nonfood-paired tokens. When tokens were delivered, their immediate exchange was required to start new trials. Accuracy was slightly higher when correct responses produced food than when correct responses produced food-paired tokens. Accuracy was significantly higher when correct responses produced food-paired tokens than when correct responses produced nonfood-paired tokens. In all of Cowles's experiments described above, the delivery of food-paired tokens functioned as a reinforcer for accuracy when exchanges for food were delayed or immediate.

Pavlovian processes. Tokens, like other conditioned reinforcers (Williams, 1991, 1994a, 1994b), may in part function to maintain responding by mediating delays between when responses occur and the exchange for backup reinforcers (Hackenberg, 2009; Jackson \& Hackenberg, 1996; Shahan, 2010). Wolfe (1936) evaluated how long responding would persist under various conditions of immediate token delivery and delayed food delivery. Four conditions 
were used: 1.) food-paired tokens were delivered immediately for a response, but exchanges for food occurred after a delay; 2.) food was earned for a response but was delivered after a delay; 3.) nonfood-paired tokens were delivered immediately for a response and food was delivered after a delay, but exchanges were not required; 4.) food-paired tokens were delivered and exchanged immediately after a response, but food was delivered after a delay. After each delayed food delivery, the delay to the next food delivery was increased. Delays were increased in this manner in each condition until a breakpoint delay was reached when responding stopped for 5 min. The longest delays were reached in the condition associated with immediate food-paired token delivery and delayed exchanges for food. Wolfe (1936) suggested that tokens functioned as a means to an end or a medium of exchange that mediated delays to reinforcement (cf. Shahan, 2010). However, delays may have also been mediated by the Pavlovian processes involved in marking (Lieberman, Davidson, \& Thomas, 1985), bridging (Rescorla, 1982), and conditioned reinforcement (Hackenberg, 2009; Williams, 1991). Immediate token delivery served to mark the response and signal impeding food delivery. The presence of tokens served to bridge the delay to food delivery. The temporal pairing of tokens with food delivery via exchanges imparted conditioned reinforcing properties to the tokens. All three of these processes were present and thus responding maintained over the longest delays in the condition that resulted in immediate token delivery and delayed exchanges for food (Hackenberg, 2009).

Generalized conditioned reinforcers. That tokens function to immediately reinforce behavior and mediate delays to the delivery of other reinforcers has long been a boon to clinicians. Since Allyon and Azrin's (1968) work with psychiatric in-patients, token economies have been one of the most successful, widely used behavioral modification techniques (Kazdin \& Bootzin, 1972; Kazdin, 1982). Token economies have been used across a variety of populations 
and locations to promote appropriate behavior and improve performance including psychiatric in-patients and others diagnosed with mental disorders (Allyon \& Azrin, 1968; Dickerson et al., 2005), at-risk youths, juvenile delinquents, and adult felons (Milan \& McKee, 1976; Phillips, 1968; Phillips, Phillips, Fixsen, \& Wolf, 1971), individuals with developmental disabilities and autism spectrum disorders (DeLeon et al., 2013; Matson \& Boisjoli, 2009), typically developed school children (Maggin et al., 2011), and in workplace settings (Fox et al., 1987). One of the advantages of using token economies is that tokens can function not just as conditioned reinforcers, but also as generalized conditioned reinforcers.

Generalized conditioned reinforcers are conditioned reinforcers that are paired with multiple backup reinforcers and thus are not as likely to be affected by changes in motivating operations (e.g., deprivation or satiation) for a single backup reinforcer (Catania, 2007). Money (a kind of token) is the most common example given for a generalized conditioned reinforcer. Human participants in laboratory settings often come into the laboratory with a long, rich history of behavior previously reinforced with money. Though having a pre-established generalized conditioned reinforcer is convenient for many research questions, it makes it difficult or precludes determining what is required to create generalized conditioned reinforcers (DeFulio, Yankelevitz, Bullock, \& Hackenberg, 2014). Understanding the use and development of generalized conditioned reinforcers is important as generalized conditioned reinforcers and conditioned reinforcement, generally, are used to explain how human behavior is reinforced and maintained (Skinner, 1953; Williams, 1994a, 1994b). Despite its importance as a concept, only a few studies have investigated the necessary and sufficient conditions to create generalized conditioned reinforcers. 
The use of token schedules of reinforcement with nonhuman animals has almost exclusively used tokens paired with only a single primary reinforcer. When tokens are only paired with a single reinforcer, a functional relation is observed between responding and the degree of deprivation for that single reinforcer (DeFulio et al., 2014; Wolfe, 1936). Wolfe (1936) deprived chimpanzees of either peanuts or water for 16 or $24 \mathrm{hr}$ prior to presenting them with a choice situation where they could respond for tokens paired with either peanuts or water. More responding occurred on the alternative associated with the tokens for the currently deprived reinforcer. This effect was greater for those chimpanzees that were deprived of the specific reinforcer for longer periods of time. DeFulio et al. (2014) found a similar effect with the responding of pigeons maintained by the delivery of food-paired or water-paired tokens. DeFulio et al. expanded on Wolfe's (1936) work by also reinforcing responding on an alternative associated with the delivery of generalized tokens that could be exchanged for either food or water. DeFulio et al. (2014) found evidence to support that the generalized tokens functioned as more effective reinforcer. Under water restriction, more responding occurred on the alternative associated with delivery of the generalized tokens than alternatives associated with food- or water-paired tokens. Also, using a behavioral-economic method of comparing reinforcers, demand curves for generalized tokens tended to be more inelastic under increasing response requirements (i.e., with greater inelasticity suggesting greater reinforcer value) than those for food- or water-paired reinforcers.

Similar results were found by researchers who systematically established tokens as generalized conditioned reinforcers for children diagnosed with developmental disabilities (Becraft \& Rolider, 2015; Moher, Gould, Hegg, \& Mahoney, 2008). In both experiments, response rates were compared when responding resulted in the delivery of either tokens paired 
with a single reinforcer (nongeneralized tokens) or tokens paired with multiple reinforcers (generalized tokens). Both the nongeneralized and generalized tokens were paired with a highly preferred edible reinforcer. Comparisons of response rates were then made under conditions of either 24-hr deprivation or satiation (i.e., prefeeding) of the highly-preferred edible. Higher response rates tended to occur when responding resulted in the delivery of the generalized tokens. This difference was greater under conditions of satiation of the highly preferred edible in part because response rates decreased for responding maintained by delivery of the nongeneralized tokens.

In the experiments by DeFulio et al. (2014) with pigeons and those by Moher et al. (2008) and Becraft and Rolider (2015) with individuals with autism spectrum disorders, pairing tokens with multiple backup reinforcers made the tokens function as generalized conditioned reinforcers. In all these experiments, the generalized tokens functioned more effectively as reinforcers than did tokens paired with a single reinforcer. Response rates were higher and persisted under greater response requirements when responding resulted in generalized token delivery response rates. Additionally, responding maintained by generalized token delivery was not affected as much by deprivation or satiation associated with a single reinforcer. Given these similarities, it is possible that the same processes involved in creating generalized conditioned reinforcers are at work in both humans and nonhuman animals. These similarities are important because the experimental analysis of behavior has long been facilitated by demonstrating that the behavior of humans and nonhuman animals are the result of shared basic behavioral processes (DeFulio et al., 2014; Hackenberg, 2009; Higgins \& Morris, 1984; Skinner, 1953).

Discriminative stimuli. Stimuli that function as reinforcers also function as discriminative stimuli. The discriminative function of reinforcers is such that some argue that 
reinforcers only have a discriminative or signaling function (Baum, 2012). This is in part because the delivery of reinforcers, whether delivered response-dependently or-independently, may be indicative of the availability of additional reinforcers (Baum, 2012; Franks \& Lattal, 1976; Reid, 1958). Reid (1958) demonstrated this is also true of token delivery. Though some have questioned the conditioned reinforcing aspects of token delivery, there is agreement that tokens have discriminative or signaling functions that serve to organize behavior around primary reinforcer deliveries (Baum, 2012; Davison \& Baum, 2006; Shahan, 2010).

One of the most common findings regarding the discriminative functions of tokens is that token schedules of reinforcement function like a chain schedule (Hackenberg, 2009). A chain schedule is a schedule of reinforcement in which reinforcers are delivered after the completion of two or more successive component schedules (i.e., links), and each component schedule is associated with a different stimulus (Catania, 2007). In token schedules of reinforcement, each successive production of a token functions like the next link of the chain. As each successive token is produced, response rates tend to increase as the opportunity to exchange those tokens for primary reinforcers is closer in time (Bullock \& Hackenberg, 2006; Foster, Hackenberg, \& Vaidya, 2001; Foster \& Hackenberg, 2004). These increasing response rates with each successive token production segment are similar to increasing response rates observed across successive links in chain schedules (Jwaideh, 1973).

Bullock and Hackenberg (2015) conducted a series of experiments to further investigate the multiple potential functions of tokens. Across three experiments, they had pigeons respond on a two-component multiple schedule. One component used a token schedule of reinforcement, and the other component used a schedule of an equivalent response requirement or frequency of reinforcer delivery. In their first experiment, Bullock and Hackenberg compared responding 
under a token schedule of reinforcement to an equivalent tandem schedule. A tandem schedule is arranged in the same manner as a chain schedule, but each component is associated with the same stimulus (Catania, 2007). In Bullock and Hackenberg's case, the tandem schedule delivered primary reinforcers after an equivalent number of responses were made, but no tokens were produced. Bullock and Hackenberg found that responding under a token schedule of reinforcement compared to an equivalent tandem schedule produced similar results to those obtained in a previous comparison of responding under chain and tandem schedules (Jwaideh, 1973). Pauses tend to be longer and response rates lower in the first few links of the token component compared to pauses and response rates in the first few links of the tandem component.

In their second experiment, Bullock and Hackenberg (2015) compared responding in a standard token schedule of reinforcement to multiple variations on a standard token economy. One variation was what they called a reverse-chain token economy. In the standard token economy, tokens were produced on a fixed-ratio (FR) 50 schedule. Each token produced signaled the completion of a link of the schedule. When four tokens were produced, an exchange period began in which each token could be exchanged for food. In the reverse-chain token economy, pigeons started with four tokens that were removed on a FR 50 schedule. When all four tokens were removed, the exchange period began. Four tokens were then illuminated and exchanged for food. In the reverse-chain token economy, each successive removal of a token signals the completion of each link of the schedule and the approaching exchange period. Bullock and Hackenberg found similar patterns of responding in the standard and reverse-chain token economies. In both token economies, after each link of the chain was completed, when exchange periods were closer in time, response rates were higher. In the standard token 
economy, the production of each token is usually conceptualized as an appetitive event, a conditioned reinforcer that also signals the completion of each link of the schedule and the approaching exchange period. By the same reasoning, in the reverse-chain token economy, the removal of each token may be conceptualized as an appetitive event, a conditioned reinforcer that also signals the completion of each link of the schedule. The discriminative and conditioned reinforcing function of the token production or removal is influenced by its relation to the operant contingencies in effect (Bullock \& Hackenberg, 2015).

In their third experiment, Bullock and Hackenberg compared responding on a standard token economy where tokens and exchange periods were produced response-dependently to a yoked schedule where tokens and exchange periods were delivered response-independently at the same rate. They also found that response rates were higher at all points under the responsedependent schedule. However, they also found that responding persisted on the yoked schedule and, much like responding on the standard token economy, responding tended to be faster on the yoked schedule when in later links of the schedule, when the exchange period was closer in time. They suggested that the tokens had eliciting functions much like stimuli used in autoshaping procedures (Ricci, 1973).

Bullock and Hackenberg stated that tokens served multiple functions — as eliciting stimuli, discriminative stimuli, and conditioned reinforcers. Bullock and Hackenberg went on to state that it is the experimental arrangements, the contingencies in effect, which define the function of the tokens in each context. Furthermore, the acknowledgement of one function did not necessarily deny the importance of the any other potential function.

Response cost. Just as the response-dependent presentation of tokens functions as an appetitive event, the response-dependent loss of tokens can function as an aversive event (Pietras 
\& Hackenberg; Raiff, Bullock, \& Hackenberg, 2008; Rasmussen \& Newland, 2008). Responsecost procedures consist of contingencies that result in the loss or removal of reinforcers dependent on a response (Azrin \& Holz, 1966). Such procedures are routinely employed to discourage dangerous or criminal behavior in everyday life (e.g., monetary fines for speeding, drunk driving, vandalism, etc.). Similar contingencies that result in the response-dependent loss of tokens have suppressed the responding of nonhuman animals (Pietras \& Hackenberg, 2005; Raiff, Bullock, \& Hackenberg, 2008), humans in laboratory settings (Critchfield et al., 2003; Pietras, Brandt, \& Searcy, 2010; Rasmussen \& Newland, 2008; Weiner, 1962), and inappropriate or maladaptive behavior in applied settings (Capriotti, Brandt, Ricketts, Espil, \& Woods, 2012; Conyers et al., 2004; Fox et al., 1987; Kazdin \& Bootzin, 1972; Kazdin, 1982).

Pietras and Hackenberg (2005) demonstrated that response-cost contingencies functioned to suppress responding in pigeons. Pigeons responded on a two component multiple schedule. In each component, tokens were produced on a variable ratio (VR) 4 (random interval [RI] 30-s) schedule. In one of the components, a FR 2 or 10 response cost contingency was imposed. Pietras and Hackenberg found that responding decreased in the response cost component, and the FR 2 response-cost contingency decreased responding to a greater degree than did the FR 10 response-cost contingency. As the opportunity to exchange tokens for food rarely happened in the response-cost component, it was possible that response rates declined because of the reduced reinforcement rate in that component.

Raiff et al. (2008) conducted a follow-up study that compared responding under a response-cost condition in which tokens were removed response-dependently to a yoked-tokenloss condition in which tokens were removed response-independently. The yoked-token-loss condition was designed to equate the frequency of token-losses and food deliveries with the 
response-cost condition. Degree of response suppression was evaluated by comparing rates of responding in the response-cost and yoked-token-loss conditions to rates of responding in a baseline standard token economy. Lower rates of reinforcement did suppress responding in both the response-cost and yoked-token-loss conditions, but greater response suppression occurred in the response-cost component. The response-dependent removal of tokens appeared to function as a punisher for pigeons (Pietras \& Hackenberg, 2005; Raiff et al., 2008) in much the same manner as response-cost contingencies do with humans (Critchfield et al., 2003; Higgins \& Morris, 1984; Pietras et al., 2010; Rasmussen \& Newland, 2008; Weiner, 1962, 1963, 1964).

Response-cost procedures have also long been used in application. Iwata and Bailey (1974) compared the effects of two different token-economy arrangements on students' performance of academic tasks and the frequency of disruptive behavior. In one arrangement, students were awarded tokens throughout a class period for not violating class rules (e.g., being off-task, disrupting other students, etc.). In another arrangement, students were given ten tokens at the beginning of a class period and lost tokens for each instance of violating the class rules. Iwata and Bailey found that both arrangements resulted in equal reductions of rule violations and off-task behavior, and that students equally preferred both arrangements. Additionally, as offtask behavior decreased, on-task behavior necessarily increased, suggesting a possible negative reinforcement effect on behavior maintained by avoiding the loss of tokens.

\section{Parameters of Token Schedules of Reinforcement}

Token schedules of reinforcement function as second-order schedules comprised of three separate but interrelated parts: 1.) A token-production schedule that determines under what conditions responding produces tokens (e.g., one token is produced on a FR 50 schedule). 2.) An exchange-production schedule that determines under what conditions opportunities to exchange 
tokens for other goods (i.e. reinforcers) occur (e.g., tokens may be exchanged every 10 min or after 5 tokens are produced). 3.) A token-exchange schedule that determines how many tokens are required to produce a reinforcer (e.g., 1, 3, or 6 tokens may be required to produce a single reinforcer). Each part of a token schedule of reinforcement contributes to the overall pattern of responding (Hackenberg, 2009).

The token-production schedule produces patterns of responding similar to those maintained by equivalent schedules that result in the delivery of primary reinforcers (e.g., food). When the responding of chimpanzees produced tokens on either FR or fixed-interval (FI) schedules, responding to produce individual tokens tended to occur in a break-and-run pattern under FR schedules and was positively accelerated under FI schedules (Kelleher 1956, 1958). Malagodi (1967a, b, c) and Bullock and Hackenberg (2006) used rats and pigeons, respectively, to extend Kelleher's work by conducting more detailed examinations of responding under FR and variable ratio (VR) token-production schedules. As with Kelleher's results, response patterns to produce individual tokens conformed to schedule-typical behavior under FR and VR schedules (Ferster \& Skinner, 1957). In addition, an inverse functional relation was observed between response requirements to produce individual tokens and response rates.

The production of individual tokens may be viewed as a unit in a larger chain of behavior controlled by the exchange-production schedule. The pattern of responding across successive token-productions generally resembles schedule-typical patterns of behavior. Webbe and Malagodi (1978) compared the responding of rats under FR 6 or VR 6 exchange-production schedules (i.e., opportunities to exchange tokens occurred when this schedule requirement was met) when each individual token was always produced on a FR 30 schedule. Webbe and Malagodi found that the VR exchange-production schedules maintained higher response rates 
than did the FR exchange-production schedules. They found that most of this difference was the result of long post-reinforcement pausing that occurred after tokens were exchanged in the FR exchange-production schedule condition. Pausing was greater in early token-production segments under the FR exchange-production schedule than in later token-production segments, resembling a break-and-run pattern of responding typical of FR performance. Foster et al. (2001) found similar patterns of behavior with pigeons across successive token-productions with a wider range of FR and VR exchange-production schedules.

Bullock and Hackenberg (2006) expanded on previous experiments (Foster et al., 2001; Webbe \& Malagodi, 1978) by examining changes in responding across combinations of small and large token-production and exchange-production schedules. Bullock and Hackenberg had pigeons respond on token-production schedules ranging from FR 25-100 and exchangeproduction schedules ranging from FR 2-8. They found an inverse functional relation between response rates and token-production schedules at each exchange-production ratio. They also found an inverse relation between response rates and exchange-production schedules at each token-production ratio. The highest response rates tended to occur when the frequency of token delivery and exchange periods was greatest. They also found that response rates tended to increase as successive tokens were produced.

In application, schedule control by the exchange-production schedule can also be observed, even when opportunities to exchange tokens are produced response-independently. Similar patterns of behavior were observed in the application of token economies at the Achievement Place (Phillips, 1968; Phillips et al., 1971). At Achievement Place, youths referred by the local juvenile court received and lost points for engaging in appropriate and inappropriate behavior, respectively. Points were exchangeable for various privileges. Phillips et al. (1971) 
noted that when exchange periods occurred every Friday, a fixed-time (FT) 1 week exchangeproduction schedule, the participating youths tended to earn fewer points early in the week. As the week passed, the youths began earning increasing numbers of points, similar to performance in interval schedules. Phillips et al. also found levels of appropriate behavior were improved more rapidly following intake at Achievement Place when youths started on a daily exchange program, FT 1 day, versus the weekly exchange program. Frequency of appropriate behavior further improved when Phillips et al. (1971) implemented a daily response requirement. This change required the youths to earn so many points each day or lose privileges for each subsequent day until the response requirement was met. By imposing an avoidance of privilegeloss contingency, Phillips et al. were able to improve levels of appropriate behavior.

Recently DeLeon et al. (2014) compared the frequency of exchange periods on the responding of individuals diagnosed with developmental disorders. In their first experiment, DeLeon et al. had participants respond in two conditions. In the distributed condition, each time the participant performed some socially significant skill (e.g., academic activity), the participant was given $30 \mathrm{~s}$ of access to a preferred activity. In the accumulated condition, the participant was given a token for completing a similar skill. The participant was required to earn 10 tokens before being given the opportunity to exchange the tokens. Each token in the accumulated conditions was worth $30 \mathrm{~s}$ of access to the preferred activity. In each condition, sessions ended when 10 tokens were earned or 5 min elapsed, not including reinforcer consumption time. DeLeon et al. found that higher response rates occurred in the accumulated condition, the condition associated with a lower frequency of exchange opportunities.

In their second experiment, DeLeon et al (2014) had participants respond on a concurrent chains choice procedure. Participants were prompted to choose either the accumulated or 
distributed condition. Following a FR 1 choice response, participants responded under the chosen condition until 10 tokens or reinforcer deliveries occurred. Preference for either condition was measured as the cumulative number of choice responses across choice opportunities. DeLeon et al also assessed how choice responses were affected by the use of either activity or edible reinforcers. More choice responses occurred on the accumulated condition alternative than on the distributed condition alternative when activity or edible reinforcers were delivered, but the difference in choice responses between alternative was smaller when edible reinforcers were used. DeLeon et al (2014) suggested some reinforcers might only function as reinforcers when they can be consumed over an extended period of time. Thus, the temporally extended consummatory behavior associated with an activity reinforcer may have increased choice responses for the accumulated condition when an activity reinforcer was delivered. The frequency of exchange opportunities can affect response rates (Bullock \& Hackenberg, 2006; Foster et al., 2001), but DeLeon et al (2014) demonstrated that the type of reinforcer (e.g., activity or edible) and the number of reinforcers that can be consumed in an exchange period can also influence response rates, though this effect was weak.

In all of these studies, the token-exchange schedule was held constant at FR $1-$ one token was exchangeable for one reinforcer. In the only study to manipulate the token-exchange schedule, Malagodi, Webbe, \& Waddell (1975) had rats respond on either a FR or FI exchangeproduction schedule in which each token was produced on a FR 20 schedule. Across conditions, the token-exchange schedule was changed such that multiple tokens were required to produce each reinforcer. Response rates were lower when more tokens were required to produce a single reinforcer. 


\section{Negative Reinforcement in Token Economies}

As token-loss functions as an aversive event, responding may also be maintained by the avoidance of token-loss. For example, municipalities may encourage health and safety behavior by imposing monetary fines that can be avoided if residents regularly mow their lawns, if drivers and passengers in cars buckle their seat belts, and if restaurants maintain clean kitchens. These contingencies are commonplace but, like most areas of aversive control, receive little attention in research (Critchfield \& Rasmussen, 2007).

In the laboratory, Weiner $(1963,1969 a)$ conducted experiments in which human participants began sessions with a maximum point total and were instructed to keep the total as high as possible. Responding was maintained on postponement schedules that avoided or escaped point-loss periods in which the total number of points was reduced rapidly. The responding of participants maintained by the avoidance or escape of these point-loss periods continued even when each response deducted one point (Weiner, 1963). Weiner (1969a) gradually increased the periods of escape produced by responding over the course of the experiment. When responding produced longer periods of escape/avoidance of the point-loss periods, responding tended to be lower.

In application, several studies have compared the relative effectiveness of token economies in which responding was maintained by token delivery or the avoidance of token-loss. Iwata and Bailey (1974) compared the effects of two different token economy arrangements on students' performance of academic tasks and frequency of disruptive behavior. In one arrangement, students were awarded tokens throughout a class period for not violating class rules (e.g., being off-task, disrupting other students, etc.). In another arrangement, students were given ten tokens at the beginning of a class period and lost tokens for each instance of violating the 
class rules. Iwata and Bailey found that both arrangements resulted in equal reductions of rule violations and off-task behavior, and students equally preferred both arrangements. Additionally, as off-task behavior decreased, on-task behavior necessarily increased. The researchers this was the result of a possible reinforcement effect on behavior maintained by avoiding the loss of tokens. Lippman \& Motta (1993) in their comparison of token-gain and token-loss contingencies suggested that using negative reinforcement contingencies to teach individuals may be beneficial when used in conjunction with positive reinforcement contingencies. Such contingencies may be adaptive for transitions to independent living situations in which mowing the lawn, doing dishes, and washing laundry may not produce immediate programmed positive reinforcers, but will avoid complaints, fines, and unsanitary living conditions.

More recently, Donaldson et al. (2014) also found that symmetrical token-gain and loss contingencies decreased disruptive behavior. For some participants, however, greater decreases were observed in the token-loss contingency condition, and more participants preferred the token-loss contingency. Additionally Donaldson et al. suggested that token-loss contingencies may be simpler to implement. A better understanding of the contingencies that operate to maintain avoidance of token-loss may serve to make these token economies more effective and give practitioners additional treatment options.

\section{Theoretical Benefits of Tokens}

The use of token schedules may also offer a unique avenue for research into the processes of positive and negative reinforcement. Researchers in the past have attempted to examine whether appetitive events (e.g. food delivery) exert the same control over behavior that aversive events (e.g., shock delivery) do. The problem with this line of research often involves asking how much food is equivalent to a shock. De Villiers (1980) attempted to quantify this relation, 
but other researchers have instead opted to use token systems and human participants to address this issue.

By using token schedules of reinforcement, contingent gains and losses can occur along the same dimension. This allows the potential of assessing the symmetry of the law of effect. Under a symmetrical law of effect equivalent losses and gains have equivalent effects on behavior. For example, Magoon and Critchfield (2008) maintained the responding of humans, mouse-clicks, on concurrent variable-cycle (VC) schedules. VC schedules are arranged similarly to variable interval (VI) schedules. They differ in that VI schedules reinforce the first response that occurs after the interval elapses; VC schedules set up an event to occur (e.g., reinforcer delivery, a scheduled shock is avoided) contingent on the first response that occurs during the interval. If the interval elapses in a VC schedule without a response occurring, the responsedependent event is cancelled (e.g., no reinforcer delivery, a scheduled shock occurs). In Magoon and Critchfield's arrangement, responding on one schedule delivered monetary gains and responding on the other avoided monetary losses of the same amount. The results conformed to expectations based on the generalized matching law. Responding occurred on either schedule as a function of the relative distribution of reinforcement. That is, when money-gain was relatively more frequent, more responding occurred on the money-gain option and vice versa. The researchers suggested that producing monetary gains and avoiding monetary losses of the same amount had similar effects on responding.

According to another theory borne from behavioral economics, loss-aversion, aversive events (e.g., money loss) exert greater control over behavior than do appetitive events (e.g., money gain) (Brosnan, Jones, Lamberth, Mareno, Richardson, \& Schapiro, 2007; Chen, Lakshminarayanan, \& Santos, 2006; Kahneman \& Tversky, 1979, 2000; Pelé, Broihanne, 
Thierry, Call, \& Dufour, 2014). Some research from behavior analysis supports this theory (Donaldson et al., 2014; Rasmussen \& Newland, 2008). Rasmussen \& Newland (2008), for example, analyzed responding of human participants, mouse-clicks, on concurrent schedules in which one alternative produced only money-gains and the other produced both money-gains and response-dependent money-losses. More responding occurred on the money-gain only alternative than would be expected if money-gains and money-losses were of equal effectiveness. The researchers suggested response-dependent money-losses exerted greater control over responding than did equivalent gains.

However, the robustness of loss aversion across other species and even within the human species is unclear (Magoon \& Critchfield, 2008; Pelé et al., 2014; Silberberg et al., 2008). Experiments examining factors that affect responding for gains and losses in a token economy may further illustrate what factors influence the appearance of loss aversion or a symmetrical law of effect. Demonstrations of responding maintained by token-gain and avoidance of token-loss in nonhuman animals may provide evidence illustrating whether loss aversion is a product of human cultural practices or a basic behavioral process (Chen et al., 2006; Harbaugh, Krause, \& Berry, 2001; Harbaugh, Krause, \& Vesterlund, 2001; Silberberg et al., 2008). Additionally, pursuing such research would shed light on how contingencies operating in a token-loss avoidance arrangement affect responding, which may have value to both basic and applied research.

This research taken together supports the idea that behavior under token schedules of reinforcement where responding produces tokens is a joint function of the token-production, exchange-production, and token-exchange schedules. It is possible that behavior maintained by the avoidance of token-losses will be affected by manipulations in similar components: the 
frequency with which token-losses are avoided, the frequency with which opportunities to exchange the tokens occur, and the token-exchange schedule in effect. Pursuing this line of research will immediately provide greater general knowledge about responding maintained by the avoidance of token-losses and conditioned reinforcers. In the long run, this research may have additional value by providing another method for further examination of the symmetrical law of effect and loss-aversion (Magoon \& Critchfield, 2008; Silberberg et al., 2008). Additionally, this research may have translational relevance by providing more information about factors that affect responding in response-cost token economies used in applied settings (Iwata \& Bailey, 1974; Donaldson et al., 2014) and in the broader culture (e.g., municipal fines).

\section{Statement of the Problem}

The experimental analysis of token systems has implications for both basic research and application. In the laboratory, tokens often are used as reinforcers for both humans and nonhuman animals (Hackenberg, 2009). In terms of application, token systems are pervasive. In everyday life they exist in the form of monetary rewards and losses. In applied settings, they are components of behavior-management plans. The response-dependent delivery and loss of tokens can reinforce and punish behavior, respectively (Hackenberg, 2009), but less research has examined the parameters that affect responding that avoids or escapes the loss of tokens (Donaldson et al., 2014; Lippman \& Motta, 1993; Magoon \& Critchfield, 2008; Weiner, 1963, 1969) even though such contingencies exist in everyday life. Laboratory research with nonhuman animals has provided more knowledge about the parameters that affect responding maintained by the delivery of tokens (Bullock \& Hackenberg, 2015; Hackenberg, 2009). Laboratory research with nonhuman animals may provide us with more knowledge about the 
parameters that affect responding maintained by the avoidance of token-losses and what conditions are necessary to establish such behavior.

Theoretically, the analysis of token systems allows for the examination of the processes of positive and negative reinforcement using the same stimulus, in which presentations and losses of tokens correspond to equivalent gains and losses of reinforcement. Thus, responding can be maintained by the response-dependent presentation of tokens and the response-dependent avoidance of the loss of tokens. Responding under token schedules of reinforcement that result in the presentation of tokens (a positive reinforcement arrangement) are a joint function of the token-production, exchange-production, and token-exchange schedules. It is not as well understood how parameters similar to the token-production, exchange-production, and tokenexchange schedules may affect responding that avoids the loss of tokens (a negative reinforcement arrangement).

This study examined whether similar factors that affect responding to produce tokens have a similar effect on responding to avoid token-loss. This study assessed how parameters similar to the token-production, exchange-production, and token-exchange schedules used in a positive reinforcement arrangement operate in a negative reinforcement arrangement. This was done to inform our understanding of responding to avoid token-loss generally. Such information may have value in future basic research comparing the influence of equivalent losses and gains on responding (Magoon \& Critchfield, 2008; Rasmussen \& Newland, 2008; Silberberg et al., 2008) and on the use of token-loss contingencies in applied settings (Donaldson et al., 2014).

\section{Experiment 1}

Responding in a token economy is a joint function of three separate but interrelated components, the first of which is the token-production schedule. Token-production schedules 
that delivered tokens more frequently maintained higher rates of responding (Bullock \& Hackenberg, 2006; Kelleher, 1958). A similar parameter may function in a token-loss avoidance arrangement, the schedule of how frequently token-losses occur. Higher rates of responding occur when shocks or point-loss are scheduled more frequently (Courtney \& Perone, 1992; de Villiers, 1972, 1974; Weiner, 1969a).

In previous studies, responding was maintained by postponement of point-loss periods (Weiner, 1963, 1969a) and VC schedules of shock (Courtney \& Perone, 1992; de Villiers, 1972, 1974; Logue \& de Villiers, 1978) and money-loss avoidance (Magoon \& Critchfield, 2008). Variable-cycle schedules allow the experimenter to eliminate the fixed temporal relation between responses and aversive events that exists in postponement schedules, but still measure the frequency of scheduled events (e.g. token-loss) and their avoidance (Anger, 1963; de Villiers, 1972). In the first experiment, responding under VC schedules of different average durations was examined to determine if higher rates of responding to avoid token-loss would occur when token-losses were scheduled more frequently.

\section{Method}

\section{Subjects}

Three White Carneau pigeons were used. Pigeons 267 and 819 were experimentally naïve at the beginning of this experiment, and Pigeon 847 had a history of responding on a variety of reinforcement schedules. Each was maintained at $80 \%$ of free-feeding weight $( \pm 15 \mathrm{~g})$ by postsession feedings occurring at least 30 min after sessions. Food used in sessions and post-session feedings consisted of Purina Nutri-Blend ${ }^{\mathrm{TM}}$ pellets. Pigeons were housed individually with free access to water and health grit in a vivarium on a 12:12 hr light/dark cycle. 


\section{Apparatus}

One operant chamber with a work area of $31 \mathrm{~cm} \mathrm{H} \mathrm{x} 30 \mathrm{~cm} \mathrm{~W}$ x $33 \mathrm{~cm} \mathrm{~L}$ was used. The work panel had three 2-cm diameter Gerbrands Co. response keys. Keys were evenly spaced horizontally across the work panel. The center key was located along the midline of the panel 7 $\mathrm{cm}$ from the ceiling with another key located $5 \mathrm{~cm}$ on either side. Keys were transilluminated by 7-W, 28-V bulbs. The center and left keys were transilluminated by a green and red light, respectively. The right key was dark and inoperative. Responding on the keys produced auditory feedback clicks. Reinforcers were delivered by raising a Gerbrands Co, model G610, hopper filled with Purina Nutri-Blend ${ }^{\mathrm{TM}}$ pellets. The hopper was located behind a 5-cm square aperture, $7 \mathrm{~cm}$ below the center key. During reinforcer deliveries, the aperture was lit by a 7-W, 28-V bulb. A 7-W, 28-V houselight was located behind a 5-cm diameter circular aperture $2 \mathrm{~cm}$ from the right side of work panel and $2 \mathrm{~cm}$ from the floor provided general illumination. A row of 7 green 40-mcd LEDs were centered $2 \mathrm{~cm}$ above the center key with each LED spaced $1 \mathrm{~cm}$ apart on either side. A ventilation fan and white noise generator provided ventilation and masked extraneous sound. A computer running Med-PC IV® software controlled experimental procedures and recorded data.

\section{Procedure}

All sessions occurred at approximately the same time each day, 7 days a week.

Pretraining. Figure 1 is a diagram of the first phase of pretraining. The 7 LEDs (tokens), the houselight, and the center (exchange) key were turned on at the start of the session. The exchange key was green. One response on the exchange key turned off the houselight, the keylight, and the right-most LED and produced $2.5 \mathrm{~s}$ access to food. This constituted a token exchange. After the left-most LED was exchanged, all seven tokens were turned on again. 
Sessions ended when 63 tokens were exchanged. Sessions continued in this way until all exchange responses occurred with short latencies $(<5 \mathrm{~s})$ following reinforcer delivery. Handshaping was used to train the key-peck response to the exchange key for Pigeons 267 and 819. Figure 2 is a diagram of the second phase of pretraining. In this phase of pretraining, the left (avoidance) key was turned on at the start of the session. The left key was red. The period when only the avoidance key was on is described hereafter as the token-loss avoidance period. When the token-loss avoidance period began, the left-most token was turned on (delivered). Once the token was delivered, a fixed-cycle (FC) 5-s schedule of token-loss avoidance began. Within the fixed cycle (i.e., interval), the first response on the avoidance key canceled a tokenloss that otherwise would occur at the end of the cycle. No stimulus change accompanied the cancelation. If a cycle elapsed without a response on the avoidance key, the token was removed by turning off the LED.

When the 5-s cycle elapsed, an exchange period began. During the exchange period, the exchange key was green. If a token remained, one response on the exchange key turned off the center keylight, houselight, and the LED and produced 2.5-s access to food. When no token remained, pecks to the exchange key produced no consequence. Exchange periods lasted for 30 $\mathrm{s}$, including reinforcer time. Tokens not exchanged by the end of the exchange period were turned off and did not carry over to the next token-loss avoidance period (cf. Pietras \& Hackenberg, 2005; Raiff et al., 2008).

During this pretraining phase, the avoidance key remained transilluminated during the exchange period. A peck to the avoidance key during the exchange period terminated the remainder of the exchange period, turned off the exchange key, and began a new token-loss avoidance period by delivering one token and initiating the 5-s cycle. 
Sessions continued until the first exchange period after at least 56 tokens were delivered ended. The second phase of pretraining continued until the pigeon canceled at least $80 \%$ of token losses across three consecutive sessions. Across the last 3 sessions of the second phase of pretraining, Pigeons 267, 819, and 847 cancelled $86.67 \%, 90.76 \%$, and $91.56 \%$ of scheduled token losses, respectively.

Figure 3 is a diagram of the third phase of pretraining and the arrangement used in the rest of Experiment 1 (described below). In this phase of pretraining, the token-loss avoidance period began with the response-independent delivery of a number of tokens and the avoidance key turning on. The number of tokens delivered at the beginning of the token-loss avoidance period was increased gradually across sessions. Initially, only a single token was delivered at the beginning of each token-loss avoidance period. Decisions to increase the number of tokens were based on visual analysis of responding on the avoidance key. If no systematic trends were observed in responding across three consecutive sessions, the number of tokens was increased. The number of tokens delivered was increased by expanding an array from which the number of tokens delivered was selected randomly without replacement. The initial array consisted only of the value 1 . This was increased to an array consisting of two values: 1 and 2 . This next was increased to an array consisting of three values: 1, 2, and 3, etc. At the end of training, a variable number of tokens were delivered by selecting randomly without replacement from an array of seven values ranging from 1-7 with an average of 4 tokens. As 56 tokens were delivered in each session, this ensured each value from the array was selected twice in each session.

Time in the token-loss avoidance period varied depending on the number of tokens delivered. Token-loss avoidance periods ended and exchange periods began when a number of cycles equal to the number of tokens delivered elapsed. For example, if one token was delivered 
at the start of the token-loss avoidance period, one 5-s cycle elapsed before the token-loss avoidance period ended and the exchange period began. If two tokens were delivered at the start of the token-loss avoidance period, two 5-s cycles elapsed before the token-loss avoidance period ended and the exchange period began, etc. Across sessions, the average number of tokens delivered at the start of the token-loss avoidance period was gradually increased. Doing this gradually leaned the frequency of exchange periods that occurred and increased the duration during which the pigeon must respond.

Once the average number of tokens delivered was increased to four, the schedule of token-loss avoidance was gradually leaned. The FC cycle changed to a VC schedule. The VC schedule consisted of 12 intervals (cycles) selected without replacement, generated from a Fleshler-Hoffman (1962) distribution with an average duration gradually increased across sessions. The values in the distribution were modified so the minimum duration was $5 \mathrm{~s} \mathrm{(cf.}$ Courtney \& Perone, 1992).

Exchange periods operated as described above, except for the differences described below. The avoidance key turned off once the exchange period began and pecks to the dark avoidance key had no programmed consequences. Exchange periods lasted until all tokens were exchanged. If no tokens remained when the exchange period was scheduled to begin, the avoidance key turned off and the exchange key flashed for $0.5 \mathrm{~s}$ before starting the next tokenloss avoidance period. Exchange periods operated in this manner for the remainder of the experiment.

Effects of VC schedules of token-loss avoidance. Table 1 lists the order of conditions and the number of sessions conducted in each condition. The VC schedule of token-loss avoidance was changed across conditions. The VC schedules were constructed as described 
above with average durations of 10, 30, and 90 s. The effects of VC 10, 30, and 90-s token-loss avoidance schedules on responding were compared across conditions. Pigeons were exposed to $\mathrm{VC}$ avoidance schedules of different average durations for a minimum of 15 sessions and until responding on the avoidance key appears stable. Stability was defined as no systematic trends over the last 5 sessions based on visual inspection. This stability criterion was used before all condition changes across the experiment.

Extinction (EXT). In the final condition, responding on the avoidance key was placed on extinction by making keypecking ineffective in avoiding token loss. Instead, token losses occurred independently of responding, on a variable time (VT) 10-s schedule, arranged in a similar manner to the VC 10-s schedule. The effect of this schedule was that each exchange period occurred with zero tokens remaining. Parameters of the schedule during this condition were, except for the response-independent token loss contingency, similar to conditions in which the VC schedules were in effect.

\section{Results and Discussion}

Figure 4 shows overall response rates on the avoidance key during the token-loss avoidance period across different VC schedules of programmed token-loss frequency. Overall response rates were determined by dividing the number of responses emitted on the avoidance key by the amount of time spent in the token-loss avoidance period. Overall response rates increased as a function of the frequency of scheduled token-losses. When responding on the avoidance key did not prevent token-losses in the EXT condition, responding dropped to zero or near zero for Pigeons 267 and 819. Responding did not entirely stop for Pigeon 847 during EXT, but response rates were at their lowest. 
Pigeon 267 was not exposed to the VC 90-s condition because overall response rates were very low in the VC 30-s condition. It seemed unlikely that further reductions in overall response rates would be observed under a VC 90-s schedule of token-loss because of a possible floor effect. Overall response rates increased in the following $\mathrm{VC} 10$-s conditions relative to the VC 30-s condition, but overall response rates in the second VC 10-s condition were lower than in the first VC 10-s condition. Response rates in one condition may have been influenced by responding in the previous condition (i.e., sequence effects). Given the low rate of responding in the second VC 10-s condition, rather than replicate the VC 30-s condition, the EXT condition was implemented. Responding in the EXT condition dropped to near zero.

Changes in response rates also were assessed in relation to the number of tokens present to evaluate whether proximity to the exchange period affected responding (Bullock \& Hackenberg, 2006; Foster et al., 2001). Local response rates on the avoidance key were calculated by dividing the number of responses on the avoidance key by the amount of time elapsed from the beginning of the token-loss avoidance period until either the right-most token was removed or the exchange period occurred, whichever came first. Figure 5 shows the average local response rates as a function of the number of tokens present from the last 5 sessions of each condition. Figure 6 shows these same data aggregated for each VC schedule for ease of comparison. Local response rates increased when fewer tokens were present. As with the overall response rates in Figure 4, local response rates increased as function of frequency of scheduled token-losses. Differences in local response rates between different VC schedules were greater when fewer tokens were present.

Figure 7 shows the percentage of scheduled token-losses that were canceled for each pigeon. This index was determined by dividing the total number of scheduled token-losses 
canceled by the total number of tokens delivered in a session (56). The percentage of scheduled token-losses canceled did not change systematically as a function of the frequency of scheduled token-losses. For Pigeons 819 and 847, there was little variation in the percentage of scheduled token-losses that were canceled across conditions. The percentage of scheduled token-losses canceled across VC 10-90-s conditions averaged 59.21\% (range, 37.50-82.14\%) for Pigeon 819 and 58.36\% (range, 46.43-75.00\%) for Pigeon 847. For Pigeon 267, the percentage of scheduled token-losses canceled in the first VC 10-s condition averaged 69.29\% (range, 53.57-73.57\%). The percentage of token-losses canceled decreased in the following VC 30-s and VC 10-s conditions to an average of $32.14 \%$ (range, $8.93-50.00 \%$ ) and $38.93 \%$ (range, 19.64-51.79\%), respectively.

Figures 8 and 9 show the frequency of exchange periods and token exchanges across conditions, respectively. The frequency of exchange periods was determined by dividing the total number of exchange periods that occurred in each session by the time spent in the token-loss avoidance period. Each token exchange was followed by food delivery. The frequency of token exchanges was determined by dividing the total number of tokens exchanged by the total session time minus food delivery time ( $2.5 \mathrm{~s} /$ food delivery). Conditions associated with more frequent scheduled token-losses were associated with more frequent exchange periods and token exchanges. The EXT condition was associated with the same frequency of exchange periods as the VC 10-s condition. Because no token-losses were canceled in the EXT condition, these exchange periods were brief (.5-s) and were not associated with token exchanges. These brief presentations of the exchange period stimuli in the EXT condition were not sufficient to maintain responding without being paired with token exchanges relative to the other conditions (Figures 4, 5, and 6). 
Overall response rates were a function of the frequency of scheduled token-losses (Fig. 4). This relation is similar to that obtained between response rates and the rate of programmed electric shock delivery under electric-shock avoidance schedules (e.g., Courtney \& Perone, 1992; de Villiers, 1972, 1974; Logue \& de Villiers, 1978). This relation is also similar to the relation between response rates and the frequency of scheduled point- or money-losses observed when responding of humans is maintained by the avoidance of point- or money-loss (Magoon \& Critchfield, 2008; Weiner, 1969a).

The relation between the overall rate of responding of pigeons and the frequency of scheduled-token losses in this experiment is similar to previous experiments in which the responding of nonhuman animals was maintained by the delivery of tokens. The rate of responding of nonhuman animals in previous experiments changed as a function of the frequency of token-deliveries (Foster et al., 2001; Malagodi 1967a, b, c). The frequency of scheduled token-losses in a token-loss avoidance arrangement appears to function in the same manner the as the frequency of token-deliveries in a token-production arrangement.

There also appeared to be some similarities in the changes in local response rates in this experiment and previous experiments in which responding was maintained by the delivery of tokens. In those previous experiments (Bullock and Hackenberg, 2006; Foster et al., 2001; Webbe \& Malagodi, 1978), response rates increased across token-production segments (i.e., the number of tokens so far produced and accumulated on the counter). There was a functional relation between the number of tokens and local response rates. This may have been because the more tokens present, the closer subjects were temporally to exchange periods and food. In this experiment, there was an inverse relation between the number of tokens and local response rates. The fewer tokens present, the closer pigeons were to exchange periods and food. The closer 
pigeons were to exchange periods and food, response rates increased (Figures 5 and 6). Bullock and Hackenberg (2006) also found that local response rates tended to be highest in those conditions associated with the most frequent token-deliveries. In the present experiments, higher local response rates occurred in the conditions associated with the more frequent scheduled token-losses.

Bullock and Hackenberg (2006) found that response rates were affected by both the token-production and exchange-production schedule. Response rates were higher when tokens and exchange periods were produced more frequently. A limitation of this experiment was that the frequency of scheduled token-losses and exchange periods varied concurrently. In Experiment 2, the effect of the frequency of exchange periods on responding was examined when the VC schedule of token-loss was held constant.

\section{Experiment 2}

This experiment investigated the effects on token loss avoidance of the second of the three variables described in the statement of the problem, the frequency of exchange periods. When responding is maintained by the delivery of tokens, response rates are higher when exchange periods occur more frequently. In previous experiments, more frequent opportunities to exchange tokens maintained higher rates of responding than did less frequent opportunities for such exchanges (Bullock \& Hackenberg, 2006; Foster et al., 2001; Waddell, Leander, Webbe, \& Malagodi, 1972; Webbe \& Malagodi, 1978; Phillips et al., 1971). Therefore in this second experiment, token-loss avoidance responding was examined as a function of the frequency of exchange periods when the VC schedule of token-loss avoidance was constant. 


\section{Subjects and Apparatus}

Three pigeons (different pigeons from those in Experiment 1) were used. Pigeon 942 was experimentally naïve at the beginning of this experiment. Pigeons 1022 and 1104 had previous experience responding on various schedules of reinforcement. Maintenance was as described in Experiment 1. The same apparatus described in Experiment 1 was used.

\section{Procedure}

Pretraining. Pretraining described in Experiment 1 was conducted. Pigeons 942, 1022, and 1104 cancelled on average $82.32,87.72$, and $87.5 \%$ of scheduled token-losses across the last three sessions of pretraining.

Effects of exchange period frequency. Table 2 lists the order of conditions and the number of sessions conducted in each condition. Pigeons responded initially on the VC 30-s avoidance schedule as described in Experiment 1. Pigeons 942 and 1104 responded on a VC 10-s schedule in later conditions following low rates of responding on the VC 30-s schedule. The stability criteria were as described in Experiment 1.

The effects of different exchange-production schedules on responding in the context of a token-loss avoidance schedule were assessed across conditions. The frequency of exchange periods was manipulated by changing the number of tokens that were delivered at the start of each token-loss avoidance period. As in Experiment 1, time in the token-loss avoidance period varied depending on the number of tokens delivered. Across conditions a fixed number (FN) of tokens were delivered at the start of each token-loss avoidance period. Either FN 2, 4, or 6 tokens were delivered at the start of each token-loss avoidance period and the token-loss avoidance period lasted until 2, 4, or 6 VC cycles elapsed. For example, when VC 30-s cycles were used, exchange periods occurred on average every 60,120, or 180 s. Once initiated, exchange periods 
operated in the same manner as described in Experiment 1 with each token exchangeable for one reinforcer.

The removal of tokens may have served as a signal of when exchange periods were about to occur. It was possible that both the frequency of exchange periods and the number of tokens present in part influenced responding. In an attempt to separate these effects, a FN 6x1 condition was conducted. In this condition, six tokens were delivered at the beginning of the token-loss avoidance period, but an exchange period occurred at the end of each VC cycle. Thus, exchange periods occurred equally often, regardless of the number of tokens present.

In each condition, sessions ended following the exchange period that occurred after at least 60 tokens had been delivered. This was done to keep the number of tokens delivered across conditions constant.

\section{Results and Discussion}

Figure 10 shows overall response rates on the avoidance key during token-loss avoidance periods across conditions. When fewer tokens were presented at the beginning of the token-loss avoidance period, exchange periods occurred more frequently, with the exception of the FN 6x1 conditions. Tokens were scheduled to be removed according to either a VC 30- or VC 10-s schedule. When token-losses occurred on a VC 30-s schedule, overall response rates for Pigeon 1022 generally increased when exchange periods occurred more often. Pigeons 942 and 1104 responded at very low overall rates and did not show consistent differences in overall response rates across conditions. When token-losses occurred on a VC 10-s schedule, overall response rates for Pigeons 942 and 1104 increased when exchange periods occurred more often. Additionally, for Pigeon 1104 overall rates of responding increased under a VC 10-s schedule compared to a VC 30-s schedule. 
Changes in local response rates also were assessed as a function of the number of tokens present to determine if proximity to the exchange period affected local response rates, as described in Experiment 1 (Bullock \& Hackenberg, 2006; Foster et al., 2001). Unlike Figures 5 and 6, Figures 11 and 12 include all responses emitted when a given number of tokens were present. This was done because across conditions, a fixed number of tokens was given at the beginning of each token-loss avoidance period, and, within a condition, the number of tokens present only changed as tokens were lost. This allowed for comparisons of local responding across conditions when tokens were first presented and as tokens were lost.

Figures 11 and 12 show average local response rates as a function of the number of tokens present. Figure 11 shows the average local response rates from the last 5 sessions of each condition. Figure 12 shows these results aggregated for each VC schedule and FN condition for ease of comparison. Similar rates of responding occurred when 6,4 , and 2 tokens were presented in the FN 6, 4, and 2 conditions, respectively. Within each condition, local response rates increased as fewer tokens were present. Exceptions were the FN 6 condition for Pigeon 1022 and the FN 6 condition for Pigeon 942 under the VC 10-s schedule, where response rates were unchanged across the number of tokens present.

Figure 13 shows the percentage of scheduled token-losses canceled across conditions. For Pigeon 942 there was little variation in this percentage under the VC 30-s schedule. Under the VC 10-s schedule, the percentage of scheduled token-losses canceled was lower in the FN 6 and FN 6x 1 conditions compared to the FN 2 condition. For Pigeon 1104, the percentage of tokenlosses canceled did not vary systematically across conditions under the VC 30-s schedule conditions. Under the VC 10-s schedule conditions, a higher percentage of scheduled token losses were canceled when exchange periods were more frequent. For Pigeon 1022, a higher 
percentage of scheduled token-losses were canceled in the when exchange periods were more frequent.

Figure 14 shows that within the VC 30-s and VC 10-s conditions, exchange periods occurred most frequently under the FN 6x1 conditions, followed by the FN 2, 4, and 6 conditions. Generally, exchange periods occurred more frequently under the VC 10-s schedule compared to the VC 30-s schedule. Of note is that the FN 2 condition under the VC 30-s schedule and the FN 6 condition under the VC 10-s schedule produced exchange periods with the same frequency.

Figure 15 shows rates of token exchanges across conditions. For Pigeon 942 there was little variation in the rate of token exchanges across conditions under the VC 30-s schedule. The rate of token exchanges increased under the VC 10-s schedule, especially in the FN 2 conditions. For Pigeon 1104, the rates of token exchanges under the VC 30-s schedule increased and then asymptoted across conditions before declining in the FN 6x 1 condition. Under the VC 10-s schedule, rates of token exchanges increased. For Pigeon 1022, rates of token exchanges were higher in the FN 2 and FN 6x1 conditions than in the FN 6 conditions.

The results of the present experiment are generally consistent with those of previous experiments (Bullock and Hackenberg 2006; Foster et al., 2001; Webbe \& Malagodi, 1978) in which responding was maintained by the delivery of tokens. In those experiments, response rates varied as function of the frequency of exchange periods. A similar relation for overall response rates was observed for Pigeon 1022 under the VC 30-s schedule and for Pigeons 942 and 1104 under the VC 10-s schedule. 
Why this functional relation between overall response rates and frequency of exchange periods was only observed for two pigeons under the VC 10-s schedule and not under the VC 30s schedule is unclear. All pigeons performed equally well under pretraining. Poor performance on canceling scheduled token-losses may be a factor. The lower percentage of scheduled tokenlosses and associated rate of token-exchanges under the VC 30-s schedule may account for Pigeon 1104's overall response rates. However, Pigeons 942 and 1022 canceled similar percentages of scheduled-token losses (Fig. 13) and engaged in similar rates of token-exchanges (Fig. 15) under the VC 30-s schedules, yet a functional relation between response rates and the frequency of exchange periods under the VC 30-s schedule was only shown for Pigeon 1022. Perhaps, under the current token-loss avoidance arrangements, the frequency of exchange periods is not a particularly potent variable for affecting overall response rates and is only effective under a narrow range of VC schedules. This range of effective VC schedules may vary by individual subjects.

It is possible that overall response rates may affected by an interaction between the VC schedule and frequency of exchange periods. Bullock and Hackenberg (2006) found an interaction between token-production and exchange-production schedules. The highest rates of responding occurred when tokens and exchange periods were most frequent. A possible interaction between the frequency of scheduled token-losses and the frequency of exchange periods in the present experiment can be evaluated by comparing the $\mathrm{FN} 2$ condition under the VC 30-s schedule and the FN 6 condition under the VC 10-s schedule in Figures 10 and 14 for Pigeons 942 and 1104. The frequency of exchange periods was similar in both conditions (Fig. 14), but overall response rates were equal to or greater in the FN 6 condition under the VC 10-s schedule (Fig 10). These results are similar to those obtained by Bullock and Hackenberg (2006) 
when responding was maintained by the delivery of tokens. Future research may be conducted to evaluate the possible interaction of the schedule of token-loss and frequency of exchange periods.

Other similarities between this experiment and those in which responding was maintained by the delivery of tokens were present the local response rates. In responding maintained by token delivery, local response rates increased as a function of the number of tokens present (Bullock \& Hackenberg, 2006; Foster et al., 2001). Under certain conditions in this experiment, local response rates were inversely related to the number of tokens present. In both cases, local response rates increased with closer proximity to exchange periods and food. Local response rates in this experiment may have increased when fewer tokens were present because when fewer tokens were present may have signaled approaching exchange periods. If this were the case, then local response rates across tokens present should be constant in the FN 6x 1 conditions (i.e. a flat function), because exchange periods occur approximately every 10 or $30 \mathrm{~s}$ depending on the VC schedule in effect. A mostly flat function of local response rates was observed only for Pigeon 942 in the FN 6x1 VC 10-s condition. Local response rates were higher when fewer tokens were present for Pigeons 1104 and 1022 in the FN 6x1 VC 10-s and VC 30-s conditions.

Local response rates in the $\mathrm{FN} 6 \times 1$ conditions may have been influenced by histories of responding in other conditions where higher rates of responding occurred when fewer tokens were present. Freeman and Lattal (1992) demonstrated that histories of reinforcement of different response rates could influence responding in conditions where reinforcement contingencies did not require specific response rates. Weiner (1969a) showed similar effects in responding maintained by avoidance of point-loss. Tokens have been suggested to have both 
reinforcing and discriminative or signaling properties (Bullock \& Hackenberg, 2015;

Hackenberg, 2009; Mazur, 2014; Shahan, 2010). It is possible that the higher local response rates in the FN 6x1 conditions when fewer tokens were present was influenced by a history of responding in previous conditions when fewer tokens present were associated with impending exchange periods. With extended time responding in the FN 6x1 condition, perhaps local response rates across tokens present would more closely resemble those of Pigeon 942.

The percentage of scheduled-losses canceled varied across conditions in this experiment (Fig. 13). These results are different from those of Experiment 1 (Fig. 7), which showed little variation in the percentage of token-losses canceled across conditions. Perhaps the variability observed in Experiment 2 was the result of delivering a fixed rather than a variable number of tokens at the beginning of each token-loss avoidance period. Future research might compare responding in these conditions more directly.

The frequency of token exchanges also varied across conditions in the present experiment (Fig. 15). In comparing these results to Figure 10, response rates generally were higher in conditions associated with more frequent token exchanges. Higher rates of token exchanges may have contributed to increasing or maintaining overall response rates. These conditions also happened to be those conditions with a greater frequency of exchange periods. Experiment 3 therefore was conducted to determine whether responding was affected by rates of food deliveries following token exchanges by manipulating the token-exchange schedule.

\section{Experiment 3}

In this experiment, the third of the three variables described in the statement of the problem, the token-exchange schedule, was investigated. Only Malagodi et al. (1975) has previously examined the effect of the token-exchange schedule on responding maintained by 
token delivery. In that study, response rates decreased as a function of the number of tokens required to produce a single reinforcer. The third experiment was designed to assess the relation between token-loss avoidance responding and the token-exchange schedule when the $\mathrm{VC}$ schedule of token-loss avoidance and the frequency of exchange periods was held constant.

\section{Subjects and Apparatus}

Pigeons 267, 819, and 847 from Experiment 1 were used in this experiment. Maintenance was as described in Experiment 1. The apparatus was as described in Experiment 1.

\section{Procedure}

Following Experiment 1, responding was reestablished and maintained on the VC 10-s token-loss avoidance schedule described in Experiment 1 (detailed in Appendix A). The same stability criteria described in Experiment 1 were used in this experiment.

Table 3 lists the order of conditions and the number of sessions conducted in each condition. The effects of different token-exchange schedules on responding in the context of a token-loss avoidance schedule were assessed across conditions. The token-exchange schedule was manipulated by changing the token-to-reinforcer exchange ratio across conditions.

Token:reinforcer ratios of 1:1,1:3, and 3:1initially were examined. The 1:3 condition was selected because satiation was considered an issue that might decrease responding. When reliable differences were not detected in the 1:3 or 3:1 conditions, ratios of 1:12 or 12:1 were used as Malagodi et al. (1975) detected the greatest changes in responding in the 12:1 token-exchange schedule.

In the 1:1 condition, each token that remained when an exchange period began was exchangeable for a single 2.5-s period of access to food as in Experiments 1 and 2. In the 3:1 and 12:1 conditions, each peck of the exchange key exchanged the right-most token and turned off 
the exchange key and houselight for .5 s. Every third or twelfth token exchanged, depending on the condition, produced a single 2.5 -s period of access to food. The number of token-exchanges to produce a reinforcer carried across exchange periods in a session. For example, in the 3:1 condition, if one exchange period ended with only 2 tokens being exchanged, the first token exchanged in the next exchange period produced a reinforcer. The number of token-exchanges carried across sessions in a similar manner. For example, in the 3:1 condition, if the session ended with 2 tokens being exchanged, the first token exchanged in the next session produced a reinforcer. Given the low percentage of token-losses that may have been canceled in a single session, carry-over of token-exchanges across days prevented effectively placing the pigeons' responding on extinction.

In the 1:3 and 1:12 conditions, each token that remained when an exchange period began was exchangeable for three or twelve 2.5-s periods of access to food. Each of these 2.5-s periods was separated by $.5 \mathrm{~s}$, during which the exchange key and houselight were off and the tokens remained on. Exchange periods otherwise operated as described in Experiments 1 and 2. Sessions continued until the end of the first exchange period that occurred after at least 56 tokens were delivered.

\section{Results and Discussion}

Figure 16 shows overall response rates on the avoidance key during token-loss avoidance periods across conditions. In this experiment, consistent effects on overall response rates were not observed as a result of changing the token-exchange schedule. Figure 17 shows local response rates as a function of the number of tokens present. These data were calculated in the same manner as in Experiment 1. Generally, local response rates were inversely related to the number of tokens present with the exception of Pigeon 267 in the 1:12 condition. 
Other variables were examined to determine possible factors that may have contributed to the lack of consistent differences in overall rates of responding. Figure 18 shows there was no systematic change in the percentage of scheduled token-losses that were canceled across conditions for each pigeon. Figure 19 shows that the frequency of exchange occurred equally frequently across conditions and pigeons. Any differences in responding that occurred could not be attributed to differences in frequency of exchange periods.

Figure 20 shows the rate of token exchanges and food deliveries across conditions and pigeons. Manipulations made did result in the programmed changes in the token-exchange schedule and associated rates of token exchanges and food deliveries. Higher rates of token exchanges than food deliveries occurred in the 3:1 and 12:1 conditions, and lower rates of token exchanges than food deliveries occurred in the 1:3 and 1:12 conditions. Despite these manipulations, consistent differences in overall response rates were not obtained.

As in the two preceding experiments, response rates were inversely related to the number of tokens present. These results are similar to results obtained with responding maintained by the delivery of tokens (Bullock \& Hackenberg, 2006; Foster et al., 2001; Malagodi et al., 1975; Webbe \& Malagodi, 1978). In both arrangements, local response rates increase with closer proximity to exchange periods and food.

Only for Pigeon 847 in the 12:1 condition were lower response rates associated with a condition where more tokens were required to produce a single food delivery. This result differs from those obtained by Malagodi et al. (1975), who showed consistently lower response rates across subjects and conditions when more tokens were required to produce a single reinforcer.

A few factors may have contributed to the lack of consistent changes in overall response rates as a function of the token-exchange schedule. For each pigeon, the percentage of scheduled 
token-losses canceled generally was less than 50\%. It was found in Experiment 2 that increasing the frequency of exchange periods increased overall response rates (Fig. 10) and the percentage of token-losses canceled (Fig. 13). Perhaps more consistent or robust effects would have been obtained if a greater percentage of token-losses were canceled.

In Malagodi et al.'s (1975) experiments, the responding of rats was maintained by the delivery of manipulable tokens (marbles) on an FR 20 schedule. Exchange periods occurred on either FR or FI exchange-production schedules. When the exchange period became available, the rats were required to carry each token to a nearby receptacle to deposit enough tokens to produce a food pellet. As the number of tokens that had to be deposited to produce a single food pellet increased from 1 to 12 , response rates declined. These systematic effects may have been facilitated by the use of manipulable tokens. Moving multiple tokens into a separate receptacle likely requires more physical effort and entails longer delays until food delivery as compared to the exchange periods used in Experiment 3. In the present experiment, nonmanipulable tokens were exchanged with a single key-peck. Another method of manipulating the token-exchange schedule besides the ratio of tokens to food could involve manipulating the amount of responding required to exchange a single token. Malagodi et al.'s (1975) rats had to engage in a sequence of responses just to make an exchange (i.e., retrieve the marble, carry it to the receptacle, then deposit it). Perhaps increasing the response requirement (e.g., from FR 1 to FR 10) to exchange each token would have produced more consistent differences in overall response rates. Additionally, each exchange was followed by either food delivery (one or multiple) or all the other stimuli that were paired with food delivery except for the raising of the hopper. The use of all these stimuli may have had conditioned reinforcing effects that attenuated the effects of changing the token-exchange schedule. 


\section{General Discussion}

Across three experiments, nonmanipulable tokens were delivered to pigeons responseindependently. These tokens were scheduled to be removed on various VC schedules. If the pigeons responded during a designated period, the upcoming scheduled token-loss was canceled. After a number of cycles equal to the number of tokens delivered elapsed, an exchange period began. In the exchange period, whatever tokens remained were exchangeable for food. Across all experiments, responding was maintained in this negative-reinforcement-based token economy by the avoidance of token-losses. Responding in positive-reinforcement-based token economies is influenced by the token-production, exchange-production, and token-exchange schedules. Similar components of this negative-reinforcement token economy were manipulated in three experiments: the frequency of scheduled token-losses, the frequency of exchange periods, and the token-exchange schedule. In Experiment 1, a functional relation was demonstrated between the rate of responding and frequency of scheduled token-losses. In Experiment 2, a functional relation was demonstrated between the rate of responding and the frequency of exchange periods. In Experiment 3, a consistent relation between the rate of responding and the ratio of tokens to food was not demonstrated, but several procedural factors may have contributed to this outcome.

Across all three experiments, an inverse functional relation was observed between the number of tokens present and the rate of responding. These results are similar to those found in previous research that examined responding maintained by token delivery across tokenproduction segments (Hackenberg, 2009; Bullock \& Hackenberg, 2015). In those experiments, response rates tended to increase as each successive token was produced. Each token produced thus functioned like a link in a chain schedule, signaling the approaching exchange period and 
primary reinforcer delivery (Bullock \& Hackenberg 2006; Foster et al., 2001). In the reversechain token economy Bullock \& Hackenberg (2015) used in their second experiment, response rates increased with the removal of each token, signaling the approaching exchange period. In the present series of experiments, response rates tended to increase when fewer tokens were present, as fewer tokens being present was associated with exchange periods being closer in time. What follows is a discussion of the results describe above, how well they align with those of previous experiments on responding maintained by token schedules of reinforcement, and the limitations of these experiments.

\section{Limitations}

The use of these or similar methods used in these experiments may facilitate further inquiry into factors that affect responding maintained by the avoidance of token-loss and aversive control generally (Critchfield \& Rasmussen, 2007). That said, further refinement of these methods would be beneficial. For example, response rates generally were low across experiments. This is common in avoidance procedures (Courtney \& Perone, 1992; DeFulio \& Hackenberg, 2007; Richardson \& Baron, 2008). For example, differences as a function of frequency of exchange periods were found in Experiment 2, but very low response rates under the VC 30-s schedule made detecting any consistent differences difficult for Pigeons 942 and 1104.

There are ways to increase response rates in a token-loss avoidance arrangement. Scheduling token-losses more frequently likely would increase overall response rates (Experiment 1). However, under the current arrangement, when token losses were scheduled to occur more frequently, exchange periods also became more frequent. These concurrent changes make evaluating the individual contribution and possible interaction of $\mathrm{VC}$ schedules and 
frequency of exchange periods to overall response rates difficult. A more thorough assessment of responding across a range of VC schedules and frequency of exchange periods may provide additional information useful in controlling behavior with negative-reinforcement-based token reinforcement.

Although all pigeons canceled greater than $80 \%$ of scheduled token-losses on average across the last three sessions of pretraining in Experiments 1 and 2, their performance generally declined in each experiment. This decline may have contributed to the inconsistent effects in Experiment 3 and under the VC 30-s schedule in Experiment 2. Changes in responding in part may have been the result of low overall rates of primary reinforcement. Canceling a greater percentage of token-losses in Experiment 3 would have led to more frequent token exchanges. This would have increased the pigeon's contact with the changes in the token-exchange schedule and perhaps would have affected overall response rates.

There may be ways to increase overall rates of responding and the percentage of scheduled token-losses canceled in the current token-loss avoidance arrangement, for example by making the VC schedule of token-losses more frequent and increasing the frequency of exchange periods. However, this may mean having to work within a relatively narrow range of schedule parameters. Although this may limit the range of schedule values that can be assessed, consistent effects can still be observed as a function of manipulating schedule values within this narrow range. Reliable differences in responding occurred across VC schedules with an average duration of 10-90 s. This is not much different from the methods of Magoon and Critchfield (2008), who used VC schedules with an average duration of 11-100 s. Other research with pigeons responding on token economies (Foster et al., 2001; Foster \& Hackenberg, 2004; Bullock \& 
Hackenberg, 2006) produced reliable and robust effects across a small range of exchangeproduction schedules (FR or VR 2-8).

\section{Functions of Tokens in the Current Token-Loss Avoidance Arrangement}

Tokens generally are conceptualized as conditioned reinforcers that acquire their reinforcing properties through repeated and reliable pairings with primary reinforcers (Hackenberg, 2009). Tokens also may have discriminative and eliciting functions as their accumulation may signal approaching opportunities for exchange for other reinforcers (Baum, 2012; Bullock \& Hackenberg, 2015; Shahan, 2010). Separating the conditioned reinforcing and discriminative/eliciting aspects of stimuli may not be entirely possible, but numerous methods attempting to do so have been investigated (Bullock \& Hackenberg, 2015; Royalty, Williams, \& Fantino, 1987; Williams 1994a, 1994b). Responding was maintained across the present series of experiments, but what the precise function of the tokens in the present series of experiments were is not clear.

There is some supporting evidence for possible conditioned reinforcing functions of the tokens in the present series of experiments. In Experiment 1, response rates changed as function of scheduled token-loss frequency. These results are similar to those of Magoon and Critchfield (2008), in which humans responded to avoid money-losses (i.e., token-losses, generalized conditioned reinforcer-losses) and response rates changed as a function of scheduled moneylosses. The results of the present series of experiments also are similar to those obtained in experiments where responding of nonhuman animals is maintained by token delivery. In those experiments (Bullock \& Hackenberg, 2006; Kelleher, 1958), response rates changed as a function of the frequency of token delivery. In all the experiments described in this paragraph, 
the consequence of responding and the putative reinforcer that maintained responding was tokendelivery or avoidance of token-loss.

Another effect of and a major advantage of using tokens as conditioned reinforcers is that they can be used in the acquisition of new behavior (DeLeon et al., 2013; Hackenberg, 2009; Kazdin \& Bootzin, 1972). After chimpanzees learned to discriminate food-paired tokens from nonfood-paired tokens, food-paired tokens were used as reinforcers for performance on a variety of different tasks (Cowles, 1937; Wolfe, 1936; Zimmerman, 1957). Appendix B describes a pilot study with a single pigeon with a history of responding in a token-loss avoidance arrangement. In one component of a two-component multiple schedule, responding on the left key resulted in the avoidance of scheduled token-losses on a VC 10-s schedule. In the other component, responding on the right key, a response that had not been reinforced in the past, resulted in the delivery of tokens on a VC 10-s schedule. Responding on the key associated with token-delivery was maintained at slightly lower rates than responding on the key associated with avoidance of tokenlosses. Response-dependent delivery of the food-paired tokens used in the present series of experiments functioned to reinforce and maintain a new response. This may indicate the possible conditioned reinforcing properties of the tokens used in the present series of experiments.

There is some supporting evidence for possible discriminative functions of the tokens in the present series of experiments. Across all three experiments, there was an inverse functional relation between local response rates and the number of tokens present. In the present series of experiments, the fewer tokens present, the closer in time exchange periods and food were. In previous experiments in which the responding of nonhuman animals was maintained by token delivery, local response rates increased as more tokens were produced (Foster et al, 2001; Bullock \& Hackenberg, 2006). As more tokens were produced, the closer in time exchange 
periods and food were. Bullock and Hackenberg (2015) found an inverse relation between the number of tokens present and local response rates in their reverse-chain schedule arrangement. Bullock and Hackenberg (2015) did not find these changes in local response rates when responding across token-production segments in token economies were compared to equivalent sections in tandem schedules. In all of these experiments, the number of tokens present served a discriminative function that signaled the temporal proximity of exchange periods and food. Regardless of how tokens were delivered or whether positive- or negative-reinforcement contingences were arranged, local response rates increased when the number of tokens signaled that exchange periods and food delivery were closer in time.

Further evidence of the discriminative function of the tokens was provided by the results described in Appendix A. Appendix A details a brief series of manipulations that showed the response-independent cancellation of scheduled token-losses and the stimuli associated with token exchange (without food delivery) were sufficient to reinstate responding. The pattern of faster responding when fewer tokens were present was also reinstated even though responding had no effect. The tokens and the stimuli that accompanied their exchange likely had discriminative functions that occasioned these patterns of responding. This is much like how the response-independent delivery of food will reinstate patterns of responding previously maintained by food (Franks \& Lattal, 1972). When food was reintroduced, responding increased further. When a VC 10-s schedule of token-loss avoidance was reimplemented, responding increased or was maintained at the current levels despite decreases in the rate of food deliveries. The response-dependent avoidance of token-loss served to increase responding, possibly as a result of a reinforcing function of avoidance of token-loss. 


\section{Negative Reinforcement}

The results from Experiments 1 and 2 are similar to previous research where responding maintained by negative reinforcement contingencies was investigated. The results from Experiment 1 are similar to previous experiments with the responding of nonhuman animals maintained by shock-avoidance (Courtney \& Perone, 1992; de Villiers, 1972, 1974) and avoidance of timeout from positive reinforcement, (DeFulio \& Hackenberg, 2007), and the responding of humans maintained by avoidance of point- or money-loss (Higgins \& Morris, 1984; Magoon \& Critchfield, 2008; Weiner, 1969a). In each of the studies cited, a direct relation between rate of responding and frequency of scheduled aversive events was demonstrated. The results from Experiment 2 are similar to those of Richardson and Baron (2008) who demonstrated a direct functional relation between rate of responding maintained by avoidance of time-out from food delivery and the frequency of food delivery. In both experiments, the functional relations described above were extended to the responding of pigeons maintained by the avoidance of token-loss.

The number of tokens may function to stimuli used in a signaled avoidance task (de Moreaes, \& Todorov, 1977; Higgins \& Morris, 1984; DeFulio \& Hackenberg, 2007). In clock schedules, a kind of signaled avoidance task (Baron \& Galizio, 1976; Field \& Boren, 1963; Grabowski \& Thompson, 1971), clock stimuli are arranged that sequentially time down (e.g., the rightmost of a string of lights is turned off) to the occurrence of an aversive event (e.g., electric shock or timeout from positive reinforcement). In clock schedules, the majority of responding tends to occur in the segment of time just before the aversive event occurs. Responding results in the clock stimuli resetting to the stimulus arrangement that is associated with the time furthest 
from the aversive event. This resetting of the clock stimuli signals the avoidance of the aversive event and may function as a conditioned reinforcer (Higgins \& Morris, 1984).

In the present series of experiments, local response rates were highest when only one token was present. When only one token was present served as a signal for the segment of time just before the exchange period occurred. If no responding occurred during the token-loss avoidance period up to that point, no token-losses would be canceled and no tokens could be exchanged for food until the next scheduled exchange period. It is possible that responding was maintained in part by avoiding a time-out from or delay to positive reinforcement (DeFulio \& Hackenberg, 2007; Richardson \& Baron, 2008) and the number of tokens present could function like clock stimuli, signaling the approach of a time-out.

However, although the number of tokens could serve to signal the approach of the exchange period, they did not function exactly like clock stimuli. Responding in the present series of experiments did not produce a programmed stimulus change and the associated conditioned reinforcement that may accompany the cancellation of a scheduled token-loss. Additionally responding canceled scheduled token-losses, leaving more tokens present even as the exchange period continued to approach. More tokens present when the exchange period occurred would degrade any association between the number of tokens present and occurrence of the exchange period.

Basic Research/Theoretical Implications. Token-production functions as a reinforcer and token-loss functions as a punisher for both humans and nonhuman animals (Hackenberg, 2009; Pietras et al., 2010; Rasmussen \& Newland, 2008). Token-loss avoidance has been shown to function as a reinforcer for humans (Magoon \& Critchfield, 2008) in past research but not for nonhuman animals. The responding of pigeons was maintained by token-loss avoidance in the 
present series of experiments, demonstrating cross-species generality of such behavior. Demonstrating cross-species generality of responding maintained in token schedules of reinforcement is relevant because this illustrates that human behavior and nonhuman behavior are the result of similar basic behavioral processes (Hackenberg, 2009; Higgins \& Morris, 1984). Previous research in which the responding of nonhuman animals was maintained on token schedules of reinforcement has provided information about the various parameters that affect responding in a token economy (Hackenberg, 2009). Understanding these parameters and conducting further research on behavior maintained by token delivery or token-loss avoidance with nonhuman animals may provide additional information the basic behavioral processes associated with responding maintained by the production of appetitive events, the avoidance of aversive events, and how those contingencies may interact.

Additionally, this work may provide a method for future research focused on examining the effects of equivalent gains and losses on behavior, thereby allowing further assessment of the symmetry of the law of effect. A symmetrical law of effect assumes that equivalent losses and gains have equivalent effects on behavior. If this were the case, then an organism ought to respond equally as much in a token-loss avoidance arrangement as in a token-production arrangement. Another perspective, loss-aversion (Kahnemann \& Tversky, 1979, 2000), supposes that losses exert greater control over behavior than equivalent gains. If this were the case, then an organism ought to respond more in a token-loss avoidance arrangement than in a tokenproduction arrangement. In the operant literature, the results of some experiments support a symmetrical law of effect (Iwata \& Bailey, 1974; Magoon \& Critchfield, 2008) and others support loss-aversion (Donaldson et al., 2014; Rasmussen \& Newland, 2008). Loss aversion is well documented in humans (Baumeister, Bratslavsky, Finkenauer, \& Vohs, 2001; Kahneman \& 
Tversky, 1979, 2000; McGraw, Larsen, Kahneman, \& Schkade, 2010; Yechiam \& Hochman, 2013), but has not been demonstrated in nonhuman animals (Silberberg et al., 2008). Future research may use this method to examine loss aversion in nonhuman animals and determine whether it is a basic behavioral process found in multiple species or perhaps something unique to humans (Chen et al., 2006; Silberberg et al., 2008).

Pilot work for such a potential examination is detailed in Appendix B. In this pilot study, the responding of a pigeon with previous experience responding in a token-loss avoidance arrangement was examined in a two-component multiple schedule. Responding in one component canceled scheduled token-losses on a VC 10-s schedule. Responding in the other component delivered tokens on a VC 10-s schedule. After 15 sessions of responding under the multiple schedules, slightly higher rates of responding occurred in the component associated with token-loss avoidance than in the component associated with token-delivery on an equivalent VC schedule. Although these results would be in line with predictions based on loss-aversion, further investigation is required to assess how much of the differences in response rates may the result of side bias or the pigeon's history of responding under the token-loss avoidance arrangement.

Similarities between responding on the two components of the multiple schedule can be seen when local response rates are examined. In Figure B2, there is functional relation between the number of tokens present and rate of responding when responding is maintained by token delivery. There is also an inverse functional relation between the number of tokens present and rate of responding when responding is maintained by token-loss avoidance. In both components, higher rates of responding occur as time to the exchange period approaches as signaled by the 
number of tokens present. These results provide some evidence for similar processes operating in both positive- and negative-reinforcement-based token economies.

\section{Conclusions}

Token schedules of reinforcement have long been an object of study in the experimental analysis of behavior and its application. Previous research with human and nonhuman animals has demonstrated that the response-dependent delivery and removal of tokens functioned as appetitive and aversive events, respectively. Laboratory research with nonhuman animals has provided more data about the parameters of token economies (i.e., token-production, exchangeproduction, and token-exchange schedules) that influence responding maintained by token delivery and the conditioned reinforcing and discriminative functions tokens serve in those arrangements (Bullock \& Hackenberg, 2015; Hackenberg, 2009). The experimental analysis of behavior has long been served by demonstrating cross-species generality of basic behavioral processes (Higgins \& Morris, 1984). The body of research on token schedules of reinforcement has shown that the basic processes that underlie common contingencies used to govern human behavior can be modeled and examined in the laboratory. Previously, responding maintained by the avoidance of token-losses had only been demonstrated in humans. The present series of experiments demonstrates that the responding of pigeons also can be maintained by the avoidance of token-losses.

The methods used in the present series of experiments already have demonstrated some of the parameters that influence responding in a token-loss avoidance arrangement (i.e., frequency of scheduled token-losses and exchange periods). These results extend and replicate the findings of previous research with the responding of humans maintained by avoidance of point- or money-loss (Magoon \& Critchfield, 2008) and the responding of nonhuman animals 
maintained by shock avoidance (de Villiers, 1972, 1974; Higgins \& Morris, 1984) to the responding of pigeons maintained by token-loss avoidance. Similar basic behavioral processes may be involved in maintaining responding in all of the experiments just mentioned. Although further refinement of the methods used in the present series of experiments may be necessary, these methods allow for further examination of factors that influence responding maintained by the avoidance of token-losses (e.g., the token-exchange schedule) and the possible functions those tokens serve.

The results and methods of the present series of experiments may inform future basic research aimed at examining a number of theoretical issues. The methods used here describe one manner of establishing responding maintained by token-loss avoidance in pigeons. Previous research describes how to establish responding maintained by the delivery of tokens in pigeons (Hackenberg, 2009). Knowing the factors affecting behavior maintained by avoidance of tokenloss and the delivery of tokens may allow for further investigation of the symmetrical law of effect, loss aversion, possible interactions of responding maintained by concurrent positive and negative reinforcement contingencies, and can inform future applications of token schedules of reinforcement. 


\section{References}

Anger, D. (1963). The role of temporal discriminations in the reinforcement of Sidman avoidance behavior. Journal of the Experimental Analysis of Behavior, 6, 477-506.

Ayllon, T., \& Azrin, N. H. (1968). The token economy: A motivational system for therapy and rehabilitation. New York: Appleton-Century-Crofts.

Azrin, N. H., \& Holz, W. C. (1966). Punishment. In W. K. Honig (Ed.), Operant behavior: Areas of research and applications (pp. 380-447). East Norwalk, CT: Appleton-CenturyCrofts.

Baron, A., \& Galizio, M. (1976). Clock control of human performance on avoidance and fixedinterval schedules. Journal of the Experimental Analysis of Behavior, 26, 165-180.

Baum, W. M. (2012). Rethinking reinforcement: Allocation, induction, and contingency. Journal of the Experimental Analysis of Behavior, 97, 101-124.

Baumeister, R. F., Bratslavsky, E., Finkenauer, C., \& Vohs, K. D. (2001). Bad is stronger than good. Review of General Psychology, 5(4), 323-370.

Becraft, J. L., \& Rolider, N. U. (2015). Reinforcer variation in a token economy. Behavioral Interventions. Advance online publication. Doi: 10.1002/bin.1401

Brosnan, S. F., Jones, O. D., Lamberth, S. P., Mareno, C., Richardson, A. S., \& Schapiro, S. J. (2007). Endowment effect in chimpanzees. Current Biology, 17, 1704-1707.

Bullock, C. E., \& Hackenberg, T. D. (2006). Second-order schedules of token reinforcement with pigeons: Implications for unit price. Journal of the Experimental Analysis of Behavior, 85, 95-106.

Bullock, C. E., \& Hackenberg, T. D. (2015). The several roles of stimuli in token reinforcement. Journal of the Experimental Analysis of Behavior, 103, 269-287. 
Byron, M. A., Shimoff, E., Catania, C. A., \& Sagvolden, T. (1977). Uninstructed human responding: Sensitivity to ratio and interval contingencies. Journal of the Experimental Analysis of Behavior, 27, 453-467.

Capriotti, M. R., Brandt, B. C., Ricketts, E. J., Espil, F. M., \& Woods, D. W. (2012). Comparing the effects of differential reinforcement of other behavior and response-cost contingencies on tics in youth with Tourette syndrome. Journal of Applied Behavior Analysis, 45, 251-263.

Catania, A. C. (2007). Learning (4 ${ }^{\text {th }}$ ed.). Cornwall-on-Hudson, NY: Sloan Publishing.

Chen, M. K., Lakshminarayanan, V., \& Santos, L. R. (2006). How basic are behavioral biases? Evidence from Capuchin monkeys trading behavior. Journal of Political Economy, 114, $517-537$.

Conyers, C., Miltenberger, R., Maki, A., Barenz, R., Jurgens, M., Sailer, A.,...Koff, B. (2004). A comparison of response cost and differential reinforcement of other behavior to reduce disruptive behavior in a preschool classroom. Journal of Applied Behavior Analysis, 37, 411-415.

Cowles, J. T. (1937). Food-tokens as incentives for learning by chimpanzees. Comparative Psychological Monographs, 12, 1-96.

Courtney, K., \& Perone, M. (1992). Reductions in shock frequency and response effort as factors in reinforcement by timeout from avoidance. Journal of the Experimental Analysis of Behavior, 58, 485-496.

Critchfield, T. S., Paletz, E. M., Macaleese, K. R., \& Newland, M. C. (2003). Punishment in human choice: Direct or competitive suppression? Journal of the Experimental Analysis of Behavior, 80, 1-27. 
Critchfield, T. S., \& Rasmussen, E. R. (2007). It's aversive to have an incomplete science of behavior. Mexican Journal of Behavior Analysis, 33, 1-5.

Davison, M., \& Baum, W. M. (2006). Do conditional reinforcers count? Journal of the Experimental Analysis of Behavior, 86, 269-283.

de Moraes, A. B., \& Todorov, J. C. (1977). Signaled free-operant avoidance of shock by pigeons pecking a key. Journal of the Experimental Analysis of Behavior, 27, 281-291.

de Villiers, P. A. (1972). Reinforcement and response rate interaction in multiple randominterval avoidance schedules. Journal of the Experimental Analysis of Behavior, 18, 499507.

de Villiers, P. A. (1974). The law of effect and avoidance: A quantitative relationship between response rate and shock-frequency reduction. Journal of the Experimental Analysis of Behavior, 21, 223-235.

de Villiers, P. A. (1980). Toward a quantitative theory of punishment. Journal of the Experimental Analysis of Behavior, 33, 15-25.

DeFulio, A., \& Hackenberg, T. D. (2007). Discriminated timeout avoidance in pigeons: The roles of added stimuli. Journal of the Experimental Analysis of Behavior, 88, 51-71.

DeFulio, A., Yankelevitz, R., Bullock, C., \& Hackenberg, T. D. (2014). Generalized conditioned reinforcement with pigeons in a token economy. Journal of the Experimental Analysis of Behavior, 102, 26-46.

DeLeon, I. G., Bullock, C. E., \& Catania, A. C. (2013). Arranging reinforcement contingencies in applied settings: Fundamentals and implications of recent basic and applied research. In G. J. Madden (Ed.) APA handbook of behavior analysis (Volume 1, pp. 483-512). Washington, D. C.: American Psychological Association. 
DeLeon, I. G., Chase, J. A., Frank-Crawford, M. A., Carreau-Webster, A. B., Triggs, M. M., Bullock, C. E., \& Jennett, H. K. (2014). Distributed and accumulated reinforcement arrangements: Evaluations of efficacy and preference. Journal of Applied Behavior Analysis, 47, 293-313.

Dickerson, F. B., Tenhula, W. N., \& Green-Paden, L. D. (2005). The token economy for schizophrenia: Review of the literature and recommendations for future research. Schizophrenia Research, 75(2-3), 405-416.

Donaldson, J. M., DeLeon, I. G., Fisher, A. B., \& Kahng, S. (2014). Effects of and preference for conditions of token earn versus token loss. Journal of Applied Behavior Analysis, 47, $537-548$.

Ferster, C. B., \& Skinner, B. F. (1957). Schedules of reinforcement. Acton, MA: Copley Publishing Group.

Field, G. G., \& Boren, J. J. (1963). An adjusting avoidance procedure with multiple auditory and visual warning stimuli. Journal of the Experimental Analysis of Behavior, 6, 537-543.

Fleshler, M., \& Hoffman, H. S. (1962). A progression for generating variable-interval schedules. Journal of the Experimental Analysis of Behavior, 5(4), 529-530.

Foster, T. A., \& Hackenberg, T. D. (2004). Unit price and choice in a token-reinforcement context. Journal of the Experimental Analysis of Behavior, 81, 5-25.

Foster, T. A., Hackenberg, T. D., \& Vaidya, M. (2001). Second-order schedules of token reinforcement with pigeons: Effects of fixed- and variable-ratio exchange schedules. Journal of the Experimental Analysis of Behavior, 76, 159-178. 
Fox, D. K., Hopkins, B. L., \& Anger, W. K. (1987). The long-term effects of a token economy on safety performance in open-pit mining. Journal of Applied Behavior Analysis, 20, 215-224.

Franks, G. J., \& Lattal, K. A. (1976). Antecedent reinforcement schedule training and operant response reinstatement in rats. Animal Learning \& Behavior, 4(4), 374-378.

Freeman, T. J., \& Lattal, K. A. (1992). Stimulus control of behavioral history. Journal of the Experimental Analysis of Behavior, 57, 5-15.

Grabowski, J., \& Thompson, T. (1971). Response patterning on an avoidance schedule as a function of time-correlated stimuli. Journal of the Experimental Analyiss of Behavior, 18, $525-534$.

Hackenberg. T. D. (2009). Token reinforcement: A review and analysis. Journal of the Experimental Analysis of Behavior, 91, 257-286.

Harbaugh, W. T., Krause, K., \& Berry, T. R. (2001). GARP for kids: On the development of rational choice behavior. American Economic Review, 91, 1539-1545.

Harbaugh, W. T., Krause, K., \& Vesterlund, L. (2001). Are adults better behaved than children? Age, experience, and the endowment effect. Economic Letters, 70, 175-181.

Higgins, S. T., \& Morris, E. K. (1984). Generality of free-operant avoidance conditioning to human behavior. Psychological Bulletin, 96(2), 247-272.

Iwata, B. A., \& Bailey, J. S. (1974). Reward versus cost token systems: An analysis of the effects on students and teacher. Journal of Applied Behavior Analysis, 7, 567-576.

Jackson, K., \& Hackenberg, T. D. (1996). Token reinforcement, choice, and self-control in pigeons. Journal of the Experimental Analysis of Behavior, 66, 29-49. 
Jwaideh, A. R. (1973). Responding under chained and tandem fixed-ratio schedules. Journal of the Experimental Analysis of Behavior, 19, 259-267.

Kahneman, D., \& Tversky, A. (1979). Prospect theory: An analysis of decisions under risk. Econometrica, 47, 263-291.

Kahneman, D., \& Tversky, A. (Eds.) (2000). Choices, values, and frames. New York: Cambridge University Press.

Kahng, S. W., Boscoe, J. H., \& Byrne, S. (2003). The use of an escape contingency and a token economy to increase food acceptance. Journal of Applied Behavior Analysis, 36, 349353.

Kazdin, A. E. (1982). The token economy: A decade later. Journal of Applied Behavior Analysis, $15,431-445$.

Kazdin, A. E., \& Bootzin, R. R. (1972). The token economy: An evaluative review. Journal of Applied Behavior Analysis, 5, 343-372.

Kelleher, R. T. (1956). Intermittent conditioned reinforcement in chimpanzees. Science, 124, 679-680.

Kelleher, R. T. (1958). Fixed-ratio schedules of conditioned reinforcement with chimpanzees. Journal of the Experimental Analysis of Behavior, 1, 281-289.

King, G. R., \& Logue, A. W. (1990). Humans' sensitivity to variation in reinforcer amount: Effects of the method of reinforcer delivery. Journal of the Experimental Analysis of Behavior, 53, 33-45.

Lieberman, D. A., Davidson, F. H., \& Thomas, G. V. (1985). Marking in pigeons: The role of memory in delayed reinforcement. Journal of Experimental Psychology: Animal Behavior Processes, 11, 611-624. 
Lippman, M. R., \& Motta, R. W. (1993). Effects of positive and negative reinforcement on daily living skills in chronic psychiatric patients in community residences. Journal of Clinical Psychology, 49, 654-662.

Logue, A. W., \& de Villiers, P. A. (1978). Matching concurrent variable-interval avoidance schedules. Journal of the Experimental Analysis of Behavior, 29, 61-66.

Maggin, D. M., Chafouleas, S. M., Goddard, K. M., \& Johnson, A. H. (2011). A systematic evaluation of token economies as a classroom management tool for students with challenging behavior. Journal of School Psychology, 49(5), 529-554.

Magoon, M. A., \& Critchfield, T. S. (2008). Concurrent schedules of positive and negative reinforcement: Differential-impact and differential-outcomes hypotheses. Journal of the Experimental Analysis of Behavior, 90, 1-22.

Malagodi, E. F. (1967a). Acquisition of the token-reward habit in the rat. Psychological Reports, 20, 1335-1342.

Malagodi, E. F. (1967b). Fixed-ratio schedules of token reinforcement. Psychonomic Science, 8, 469-470.

Malagodi, E. F. (1967c). Variable-interval schedules of token reinforcement. Psychonomic Science, 8, 471-472.

Malagodi, E. F., Webbe, F. M., \& Waddell, T. R. (1975). Second-order schedules of token reinforcement: Effects of varying the schedule of food presentation. Journal of the Experimental Analysis of Behavior, 24, 173-181.

Matson, J. L., \& Biosjoli, J. A. (2009). The token economy for children with intellectual disability and/or autism: A review. Research in Developmental Disabilities, 30(2), 240248. 
Mazur, J. E. (2014). Rats' choices with token stimuli in concurrent variable-interval schedules. Journal of the Experimental Analysis of Behavior, 102, 198-212.

McGraw, A. P., Larsen, J. T., Kahneman, D., \& Schkade, D. (2010). Comparing gains and losses. Psychological Science, 21, 1438-1445.

Milan, M. A., \& McKee, J. M. (1976). The cellblock token economy: Token reinforcement procedures in a maximum security correctional institution for adult male felons. Journal of Applied Behavior Analysis, 9, 253-275.

Moher, C. A., Gould, D. D., Hegg, E., \& Mahoney, A. M. (2008). Non-generalized and generalized conditioned reinforcers: Establishment and validation. Behavioral Interventions, 23, 13-38.

Pelé, M. Broihanne, M. H., Thierry, B., Call, J., \& Dufour, V. (2014). To bet or not to bet? Decision-making under risk in non-human primates. Journal of Risk and Uncertainty, 49(2), 141-166.

Phillips, E. L. (1968). Achievement place: Token reinforcement procedures in a home-style rehabilitation setting for "pre-delinquent” boys. Journal of Applied Behavior Analysis, 1, 213-223.

Phillips, E. L., Phillips, E. A., Fixsen, D. L., \& Wolf, M. M. (1971). Achievement place: Modification of the behaviors of pre-delinquent boys within a token economy. Journal of Applied Behavior Analysis, 4, 45-59.

Pietras, C. J., Brandt, A. E., \& Searcy, G. D. (2010). Human responding on random-interval schedules of response-cost punishment: The role of reduced reinforcement density. Journal of the Experimental Analysis of Behavior, 93, 5-26. 
Pietras, C. J., \& Hackenberg, T. D. (2005). Response-cost punishment via token loss with pigeons. Behavioural Processes, 69, 343-356.

Raiff, B. R., Bullock, C. E., \& Hackenberg, T. D. (2008). Response-cost punishment with pigeons: Further evidence of response suppression via token loss. Learning \& Behavior, 36 (1), 29-41.

Rasmussen, E. B., \& Newland, M. C. (2008). Asymmetry of reinforcement and punishment in human choice. Journal of the Experimental Analysis of Behavior, 89, 157-167.

Reid, R. L. (1958). The role of the reinforcer as a stimulus. British Journal of Psychology, 49, 202-209.

Rescorla, R. A. (1982). Effect of a stimulus intervening between CS and US in autoshaping. Journal of the Experimental Psychology: Animal Behavior Processes, 8, 131-141.

Ricci, J. A. (1973). Key pecking under response-independent food presentation after long simple and compound stimuli. Journal of the Experimental Analysis of Behavior, 19, 509-516.

Richardson, J. V., \& Baron, A. (2008). Avoidance of timeout from response-independent food: Effects of delivery rate and quality. Journal of the Experimental Analysis of Behavior, 89, 169-181.

Royalty, P., Williams, B. A., \& Fantino, E. (1987). Effects of delayed conditioned reinforcement in chain schedules. Journal of the Experimental Analysis of Behavior, 47, 41-56.

Shahan, T. A. (2010). Conditioned reinforcement and response strength. Journal of the Experimental Analysis of Behavior, 93, 269-289.

Silberberg, A., Roma, P. G., Huntsberry, M. E., Warren-Boulton, F. R., Sakagami, T., Ruggiero, A. M., \& Suomi, S. J. (2008). On loss aversion in capuchin monkeys. Journal of the Experimental Analysis of Behavior, 89, 145-155. 
Skinner, B. F. (1953). Science and Human Behavior. New York: The Free Press.

Waddell, T. R., Leander, J. D., Webbe, F. M., \& Malagodi, E. F. (1972). Schedule interactions in second-order fixed-interval (fixed-ratio) schedules of token reinforcement. Learning and Motivation, 3, 91-100.

Webbe, F. M., \& Malagodi, E. F. (1978). Second-order schedules of token reinforcement: Comparisons of performance under fixed-ratio and variable-ratio exchange schedules. Journal of the Experimental Analysis of Behavior, 30, 219-224.

Weiner, H. (1962). Some effects of response cost upon human operant behavior. Journal of the Experimental Analysis of Behavior, 5, 201-208.

Weiner, H. (1963). Response cost and the aversive control of human operant behavior. Journal of the Experimental Analysis of Behavior, 6, 415-421.

Weiner, H. (1964). Response cost and fixed-ratio performance. Journal of the Experimental Analysis of Behavior, 7, 79-81.

Weiner, H. (1969a). Conditioning history and the control of human avoidance and escape responding. Journal of the Experimental Analysis of Behavior, 12, 1039-1043.

Weiner, H. (1969b). Controlling human fixed-interval performance. Journal of the Experimental Analysis of Behavior, 12, 349-373.

Williams, B. A. (1991). Marking and bridging versus conditioned reinforcement. Animal Learning \& Behavior, 19, 264-269.

Williams, B. A. (1994a). Conditioned reinforcement: Experimental and theoretical issues. The Behavior Analyst, 17, 261-285.

Williams, B. A. (1994b). Conditioned reinforcement: Neglected or outmoded explanatory construct? Psychonomic Bulletin \& Review, 1, 457-475. 
Wolfe, J. B. (1936). Effectiveness of token rewards for chimpanzees. Comparative Psychological Monographs, 12, 1-72.

Yechiam, E., \& Hochman, G. (2013). Loses as modulators of attention: Review and analysis of the unique effects of losses over gains. Psychological Bulletin, 139(2), 497-518.

Zimmerman, D. W. (1957). Durable secondary reinforcement: Method and theory. Psychological Review, 64(6), 373-383. 
Table 1

Order of Conditions and Number of Sessions in Experiment 1

\begin{tabular}{|c|c|c|}
\hline Subject & Condition & Sessions \\
\hline \multirow[t]{4}{*}{267} & $\mathrm{VC} 10 \mathrm{~s}$ & 22 \\
\hline & VC $30 \mathrm{~s}$ & 25 \\
\hline & $\mathrm{VC} 10 \mathrm{~s}$ & 45 \\
\hline & EXT & 16 \\
\hline \multirow[t]{6}{*}{819} & $\mathrm{VC} 10 \mathrm{~s}$ & 15 \\
\hline & VC $30 \mathrm{~s}$ & 23 \\
\hline & VC $90 \mathrm{~s}$ & 17 \\
\hline & VC $30 \mathrm{~s}$ & 47 \\
\hline & $\mathrm{VC} 10 \mathrm{~s}$ & 22 \\
\hline & EXT & 17 \\
\hline \multirow[t]{6}{*}{847} & $\mathrm{VC} 10 \mathrm{~s}$ & 15 \\
\hline & VC $30 \mathrm{~s}$ & 19 \\
\hline & VC $90 \mathrm{~s}$ & 16 \\
\hline & VC $30 \mathrm{~s}$ & 18 \\
\hline & $\mathrm{VC} 10 \mathrm{~s}$ & 31 \\
\hline & EXT & 42 \\
\hline
\end{tabular}


Table 2

Order of Conditions and Number of Sessions in Experiment 2

\begin{tabular}{|c|c|c|c|}
\hline Subject & VC Schedule & Condition & Sessions \\
\hline \multirow[t]{11}{*}{942} & VC $30 \mathrm{~s}$ & FN 2 & 16 \\
\hline & & $\mathrm{FN} 4$ & 18 \\
\hline & & FN 6 & 21 \\
\hline & & $\mathrm{FN} 4$ & 22 \\
\hline & & FN 2 & 18 \\
\hline & & FN 6 & 15 \\
\hline & & FN 2 & 18 \\
\hline & $\mathrm{VC} 10 \mathrm{~s}$ & FN 6 & 19 \\
\hline & & FN 2 & 18 \\
\hline & & FN 6 & 49 \\
\hline & & FN 6x1 & 20 \\
\hline \multirow[t]{10}{*}{1022} & VC $30 \mathrm{~s}$ & FN 2 & 15 \\
\hline & & $\mathrm{FN} 4$ & 16 \\
\hline & & FN 6 & 15 \\
\hline & & $\mathrm{FN} 4$ & 20 \\
\hline & & FN 2 & 16 \\
\hline & & FN 6 & 17 \\
\hline & & FN 2 & 16 \\
\hline & & FN 6 & 19 \\
\hline & & FN 6x1 & 39 \\
\hline & & FN 2 & 17 \\
\hline 1104 & VC $30 \mathrm{~s}$ & FN 6 & 24 \\
\hline
\end{tabular}


FN 4

FN 2

FN 6

FN 6x 1

VC $10 \mathrm{~s}$
44

19

34

16

FN 6x1

FN 6

25

FN 2

21

FN 6 
Table 3

Order of Conditions and Number of Sessions in Experiment 3

\begin{tabular}{|c|c|c|}
\hline Subject & $\begin{array}{c}\text { Condition } \\
\text { (Token:Food) }\end{array}$ & Sessions \\
\hline \multirow[t]{5}{*}{267} & $1: 1$ & 36 \\
\hline & $3: 1$ & 16 \\
\hline & $1: 3$ & 18 \\
\hline & $1: 1$ & 18 \\
\hline & $1: 12$ & 22 \\
\hline \multirow[t]{5}{*}{819} & $1: 1$ & 19 \\
\hline & $1: 3$ & 19 \\
\hline & $3: 1$ & 15 \\
\hline & $1: 3$ & 30 \\
\hline & $12: 1$ & 30 \\
\hline \multirow[t]{5}{*}{847} & $1: 1$ & 24 \\
\hline & $1: 3$ & 28 \\
\hline & $3: 1$ & 27 \\
\hline & $12: 1$ & 17 \\
\hline & $1: 1$ & 17 \\
\hline
\end{tabular}




\section{Exchange Training}

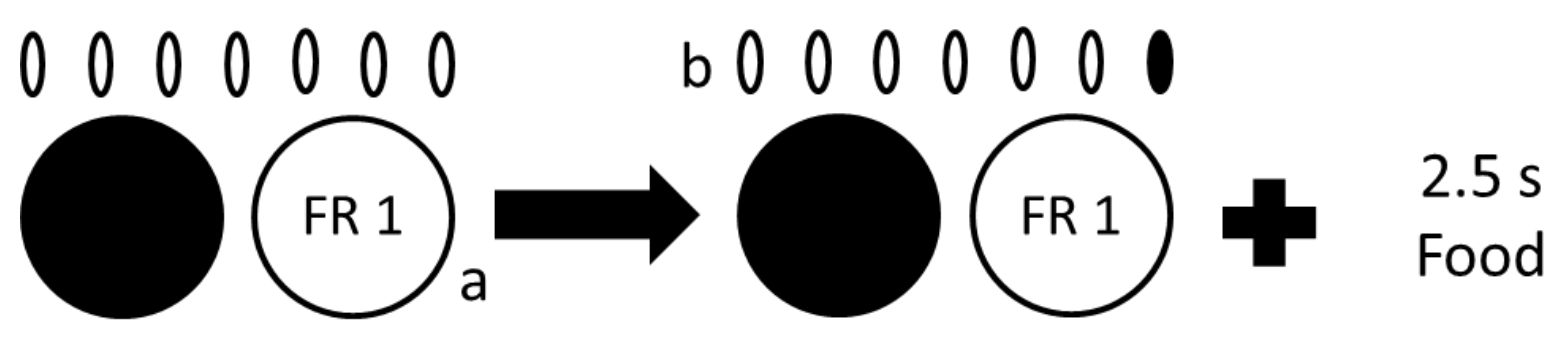

Figure 1. Diagram of the first phase of pre-training. Sessions began with 7 tokens being illuminated. Responding on the exchange key (a) exchanged (i.e., turned off) the right-most token and produced access to food (b). 


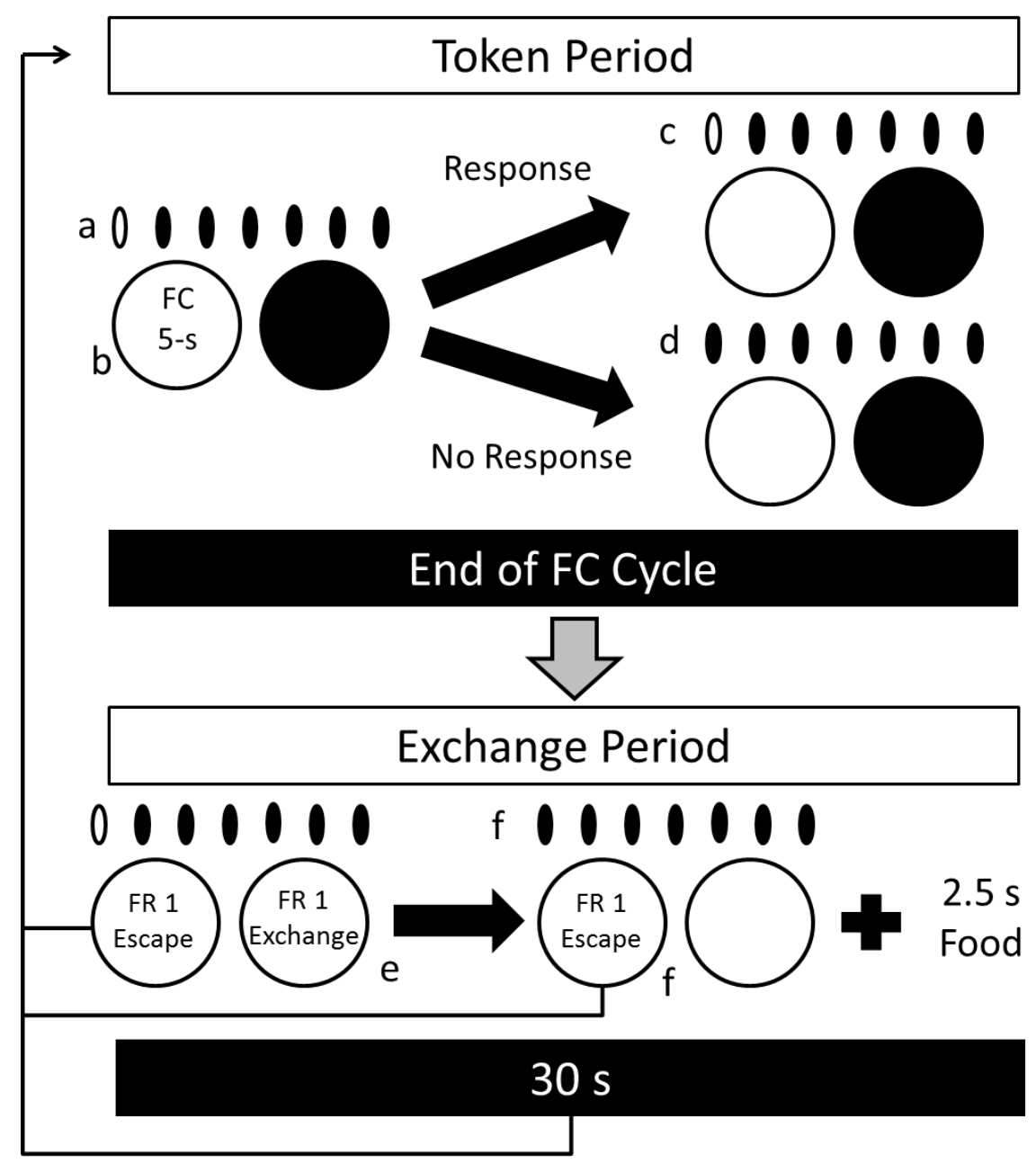

Figure 2. Diagram of the second phase of pre-training. The token-loss avoidance period began with one token being delivered (a). Responding on the avoidance key (b) avoided token-loss on a FC 5-s schedule (c). If no response occurred on the avoidance key, the token was removed at the end of the cycle (d). Exchange periods began at the end of the FC 5-s cycle. If a token remained, responses on the exchange key (e) exchanged (i.e., turned off) a token and produced access to food. Exchange periods lasted for $30 \mathrm{~s}$ before removing any remaining tokens and returning to the token-loss avoidance period. A response on the avoidance key during the exchange period (f) escaped the remainder of the 30-s exchange period and returned to the token-loss avoidance period. 


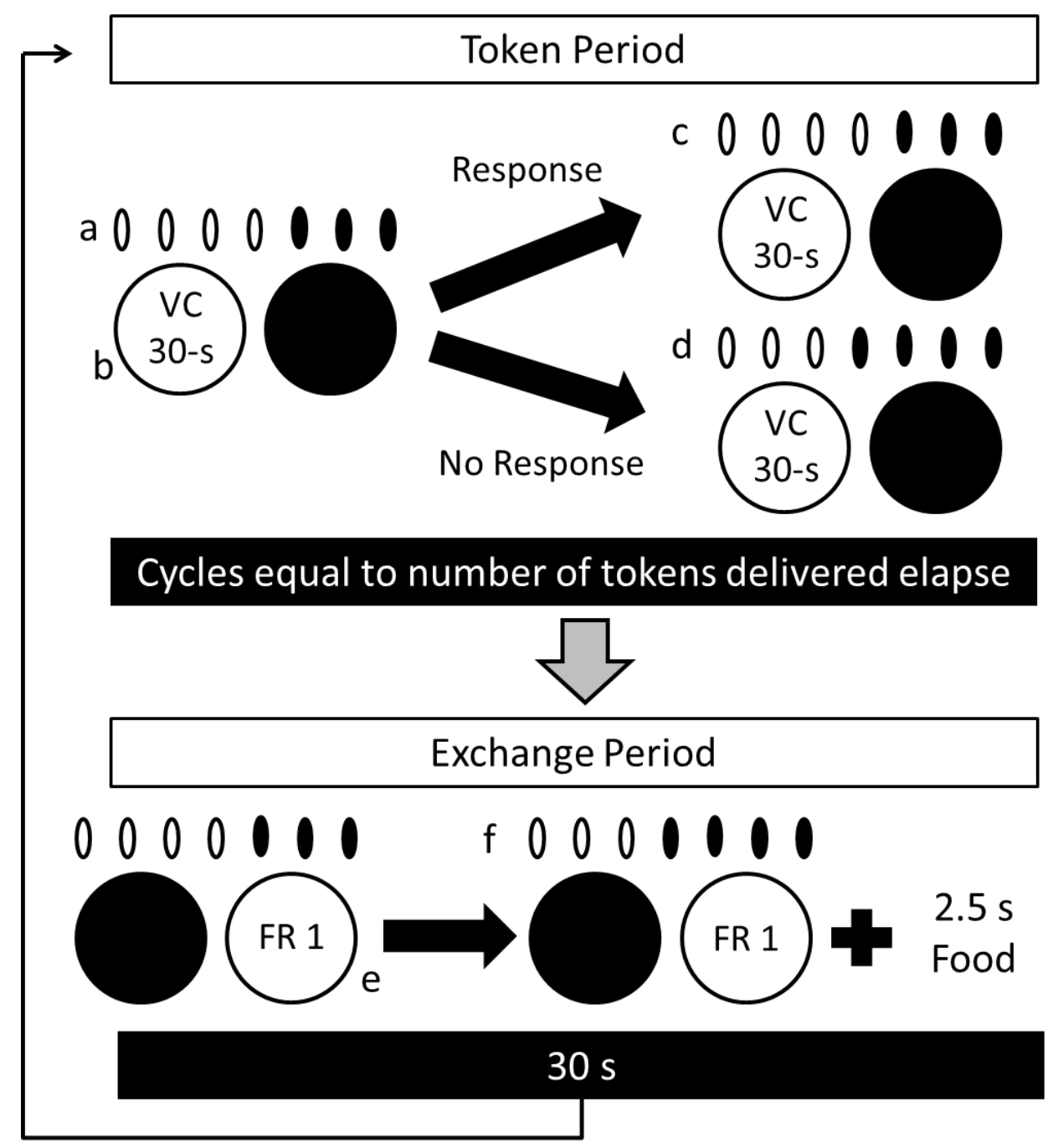

Figure 3. Diagram of the general procedure across the present series of experiments. The tokenloss avoidance period will begin with a number of tokens being delivered at the start of the token-loss avoidance period (a). Responding on the avoidance key (b) will avoid token-loss on a cycle schedule (here VC 30-s) (c). If no responding occurs on the avoidance key, a token will be removed at the end of the cycle (d). Exchange periods will begin when a number of cycles equal to the number of tokens delivered elapses. If no tokens remain, the exchange key will flash for .5 $\mathrm{s}$ before returning to the token-loss avoidance period. If any tokens remain, responding on the exchange key (e) will exchange one token and produce access to food (f). Exchange periods will last until all tokens are exchanged. 


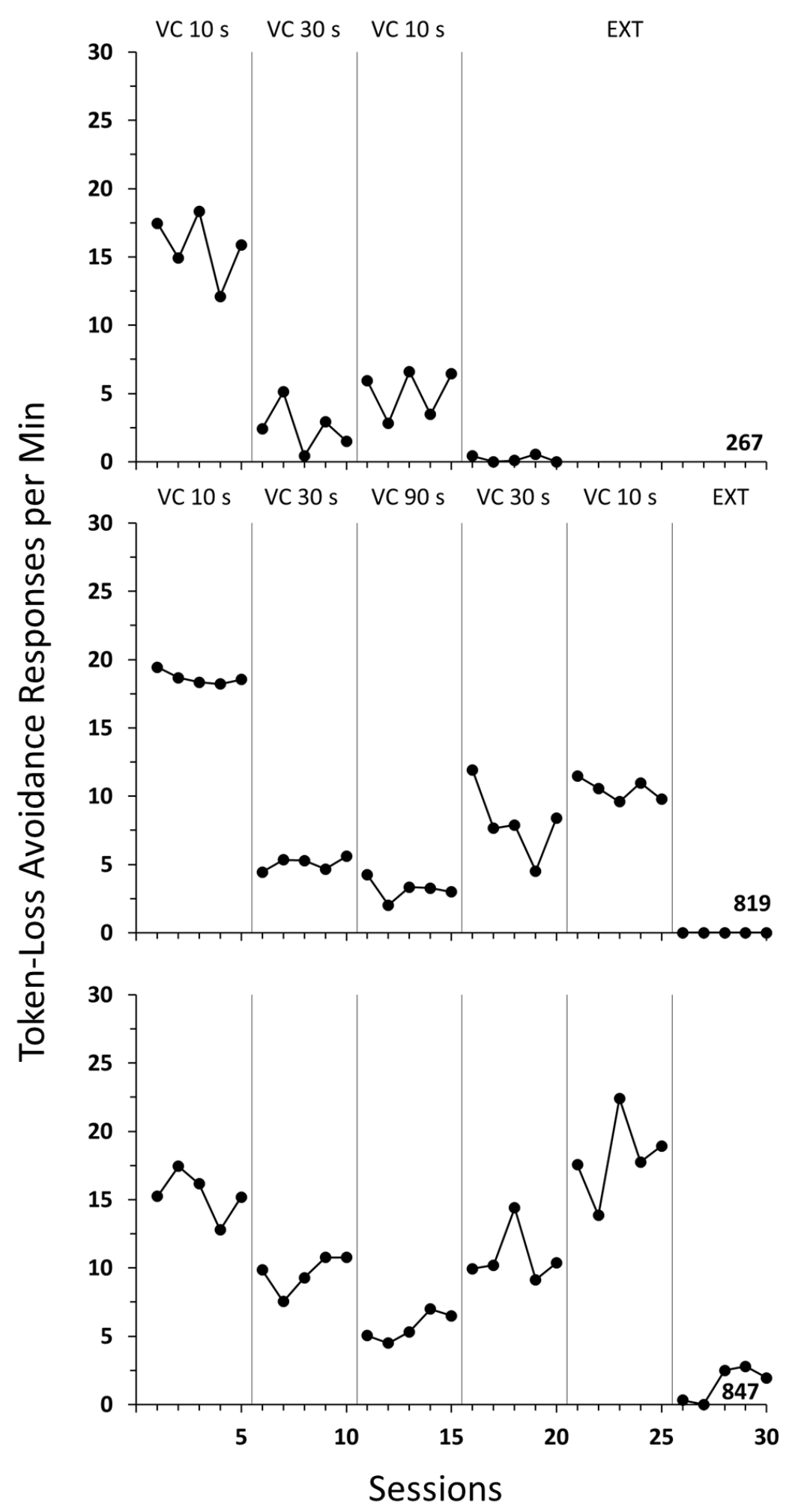

Figure 4. Overall response rates on the avoidance key across the last 5 sessions in each condition of Experiment 1. Labels indicate schedule of token-loss frequency in effect. 


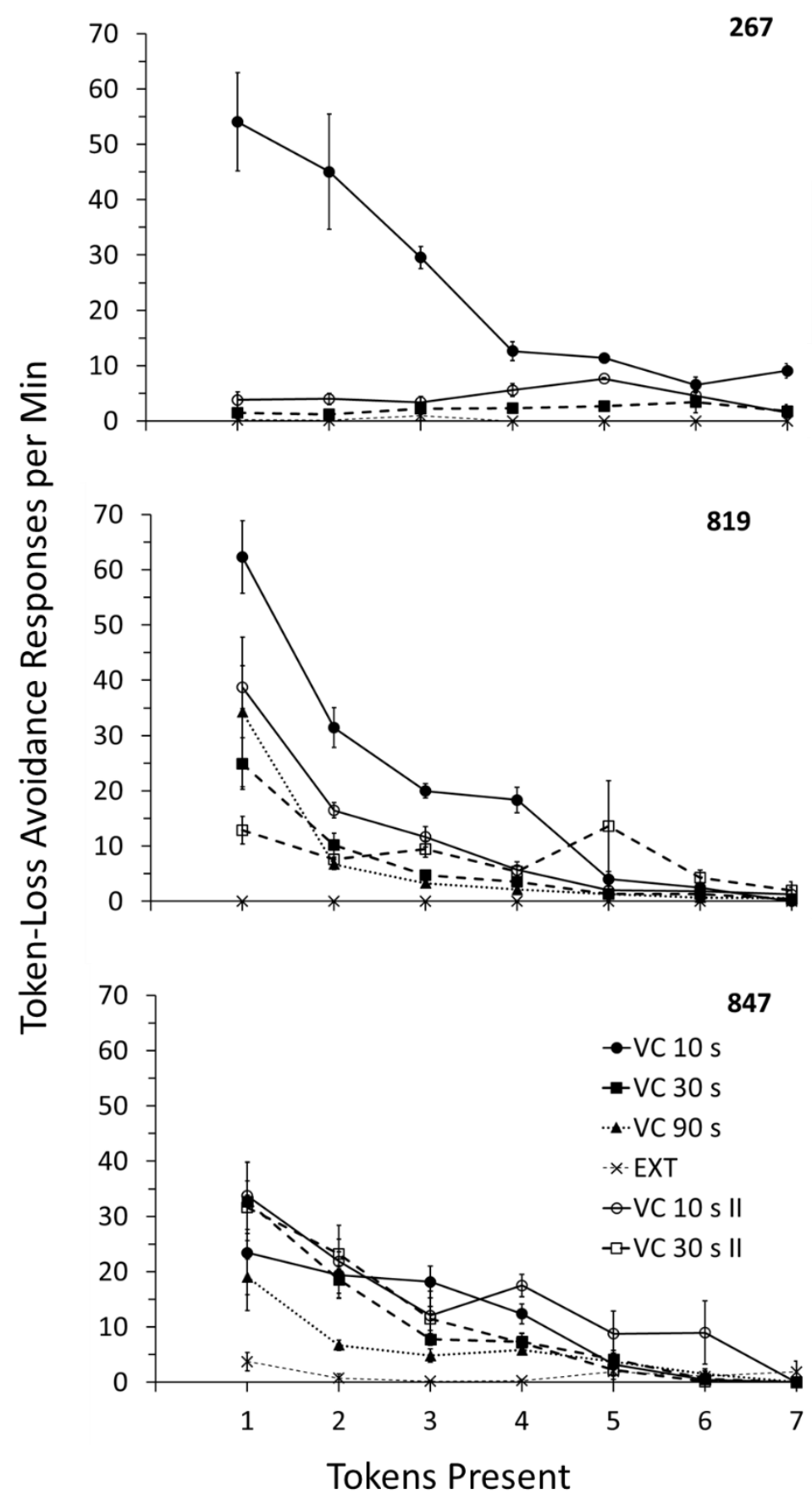

Figure 5. Local response rates on the avoidance key as a function of the number of tokens present in the last 5 sessions of each condition of Experiment 1. Data points represent responding between token delivery and the removal of a single token or the beginning of the exchange period, whichever occurs first. Error bars represent \pm 1 standard error. 


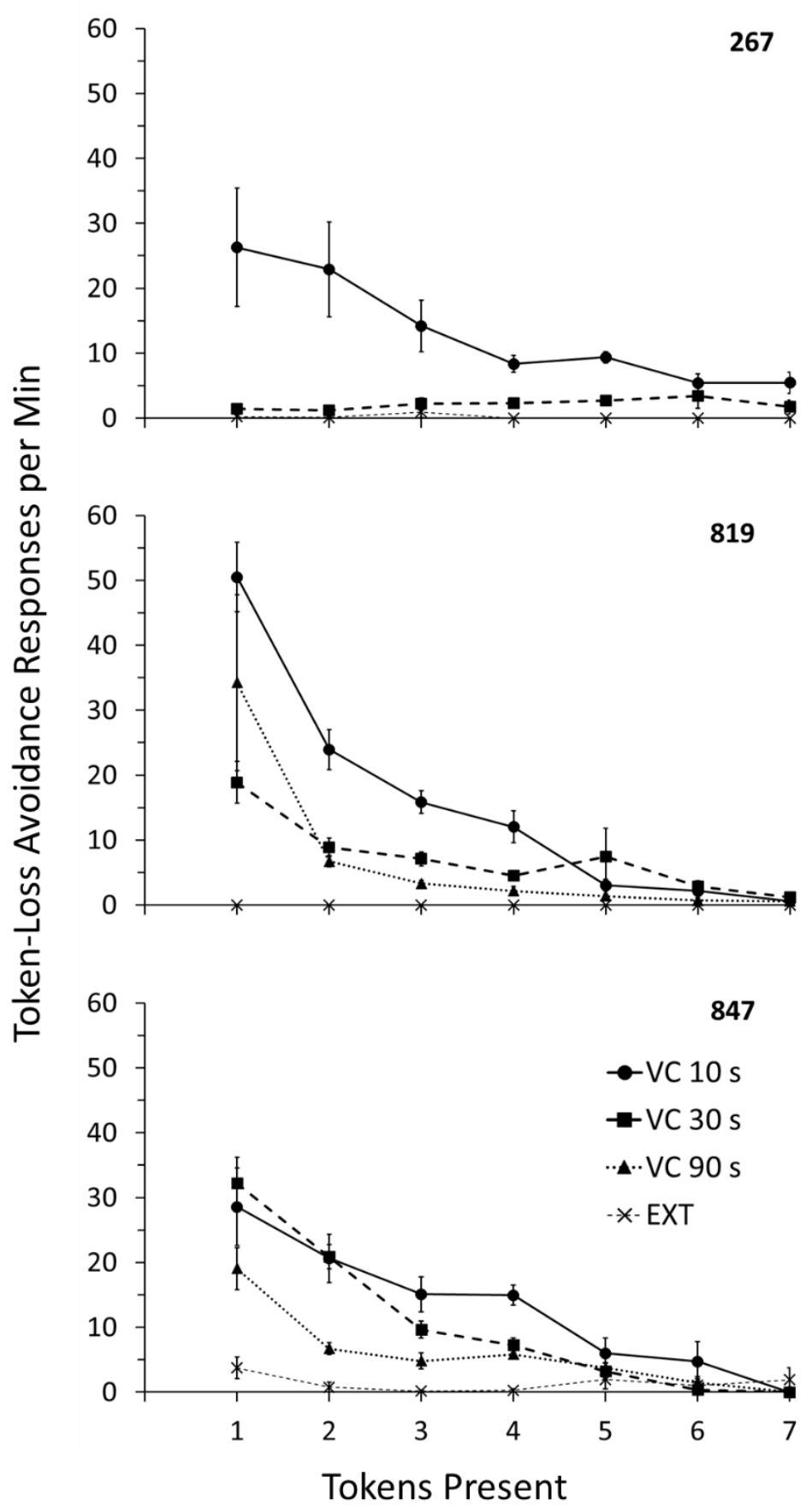

Figure 6. Aggregate local response rates on the avoidance key as a function of the number of tokens present in the last 5 sessions of each schedule of token-loss frequency in Experiment 1. Data points represent responding between token delivery and the removal of a single token or the beginning of the exchange period, whichever occurs first. Error bars represent \pm 1 standard error. 


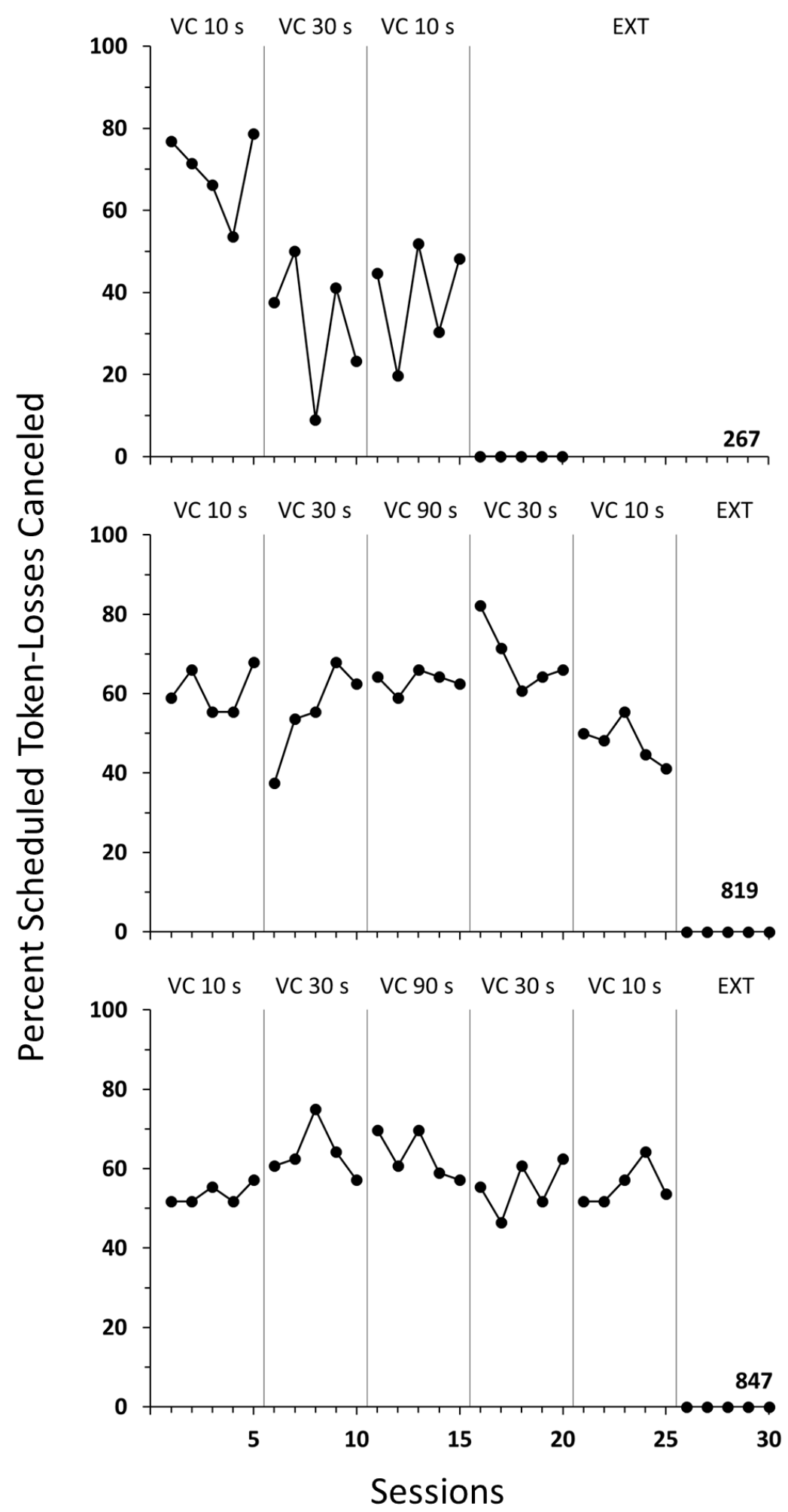

Figure 7. Percent of scheduled token-losses canceled across the last 5 sessions in each condition of Experiment 1. Labels indicate schedule of token-loss frequency in effect. 


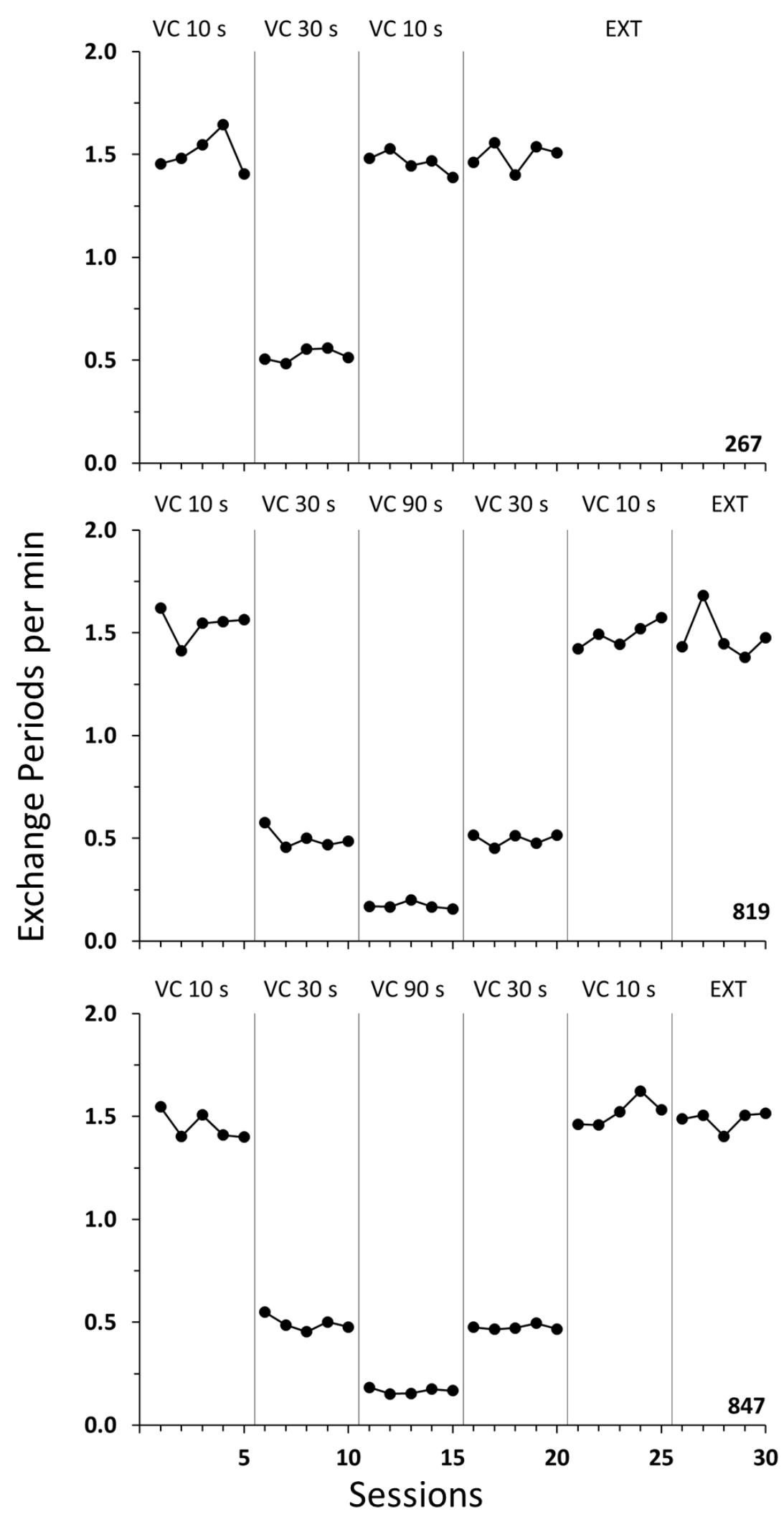

Figure 8. Rate of exchange periods across conditions of different VC schedules of token-loss. 


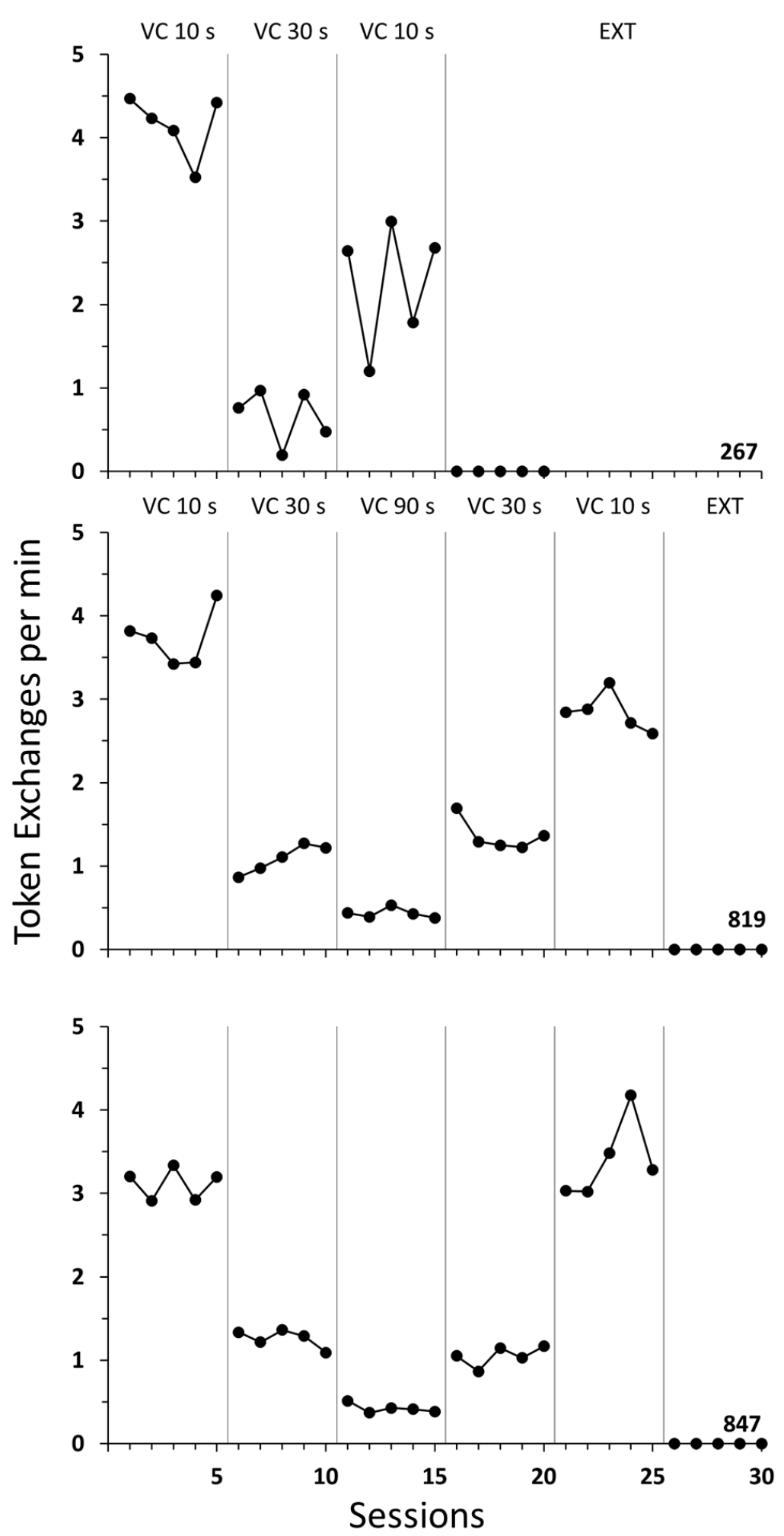

Figure 9. Rate of food deliveries across conditions of different VC schedules of token-loss. Each token exchanged resulted in one 2.5 -s access to food. 


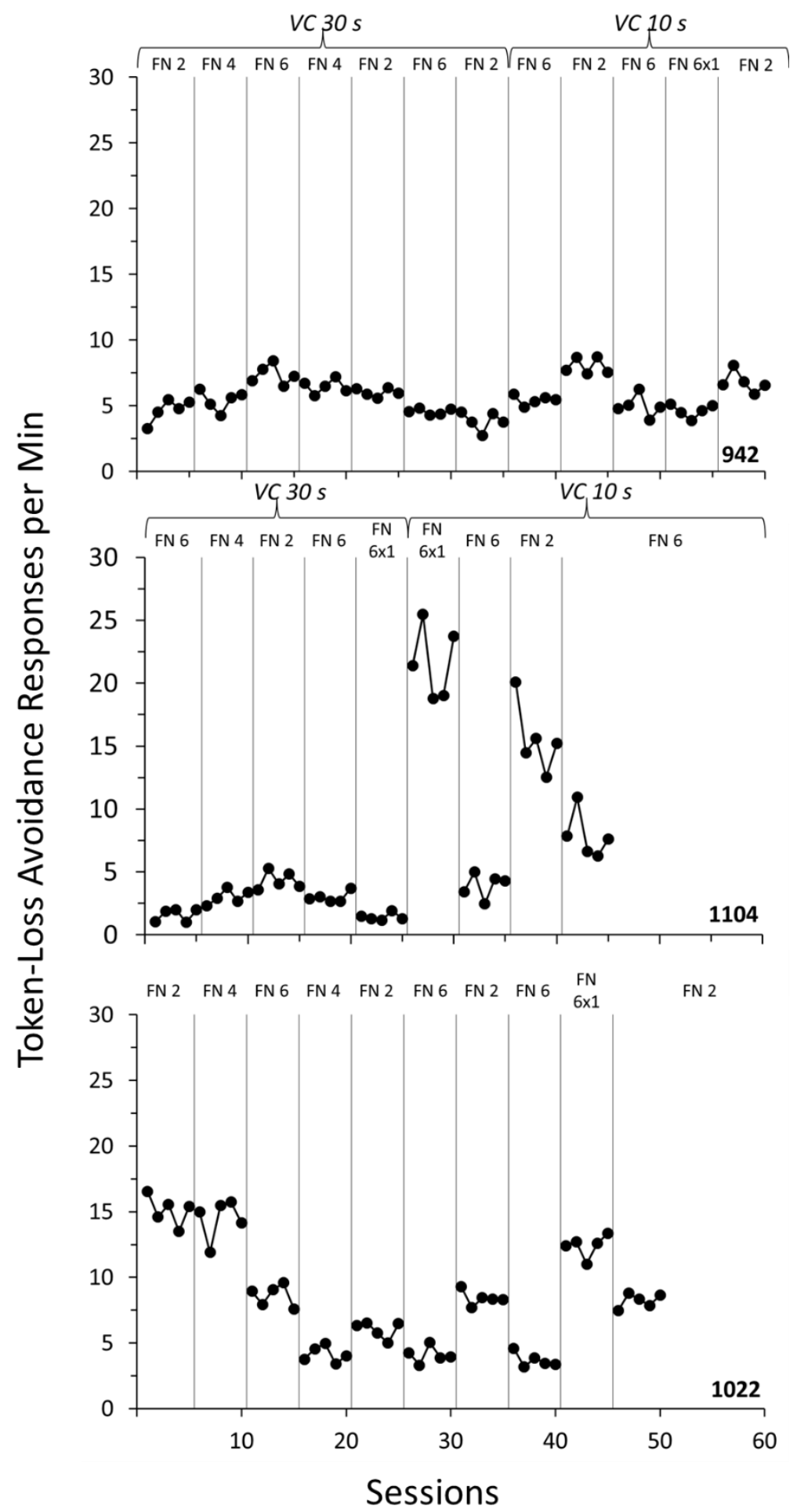

Figure 10. Overall response rates across the last 5 sessions of each condition in Experiment 2. Labels indicate the schedule of token-loss frequency and the number of tokens presented at the beginning of each token-loss avoidance period. 


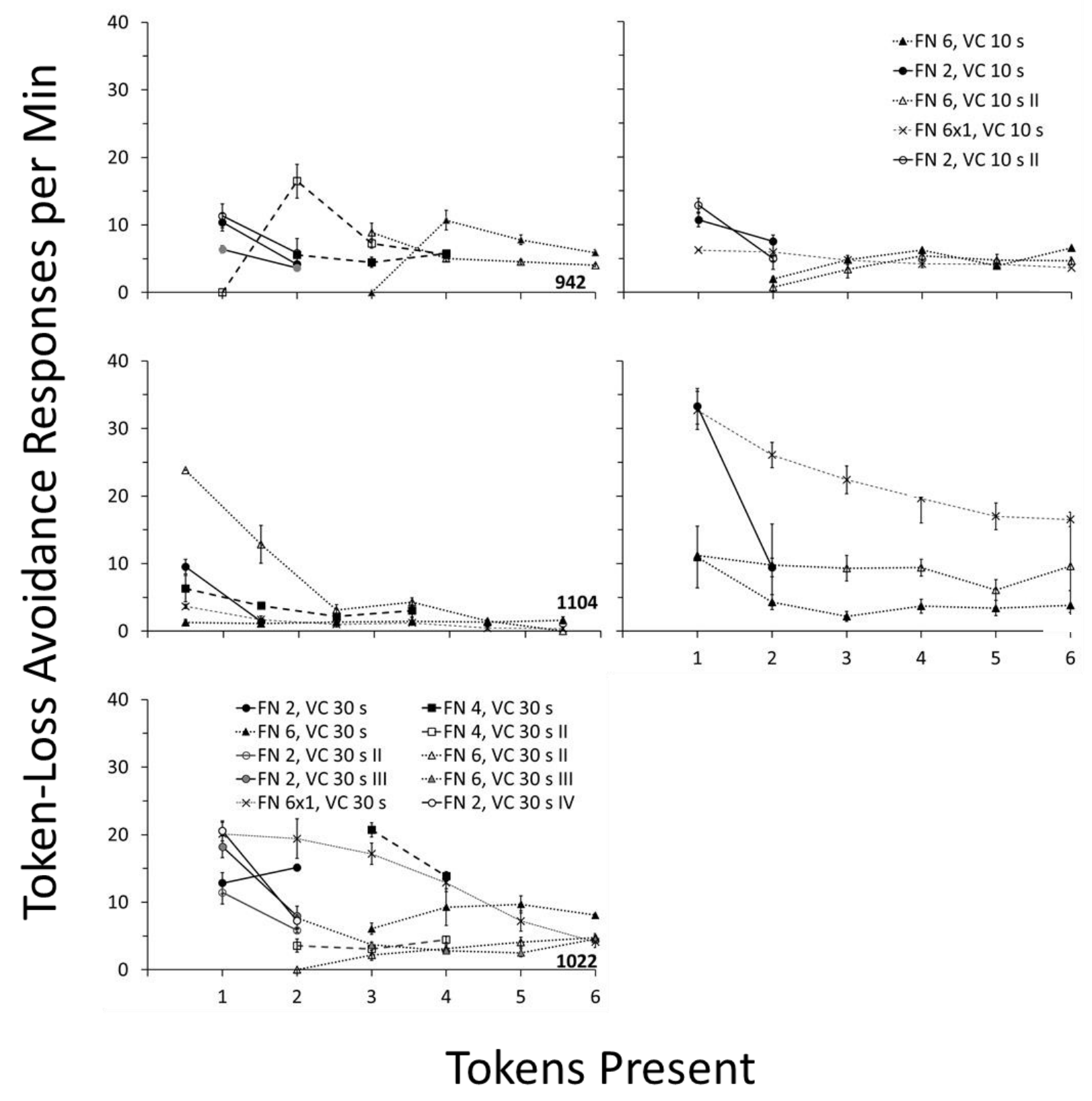

Figure 11. Local response rates on the avoidance key as a function of the number of tokens present in the last 5 sessions of each condition in Experiment 2. A VC 30-s and VC 10-s schedule of token-loss frequency was in effect for graphs in the left and right columns, respectively. Data points represent responding between token delivery and the beginning of the exchange period. Error bars represent \pm 1 standard error. 


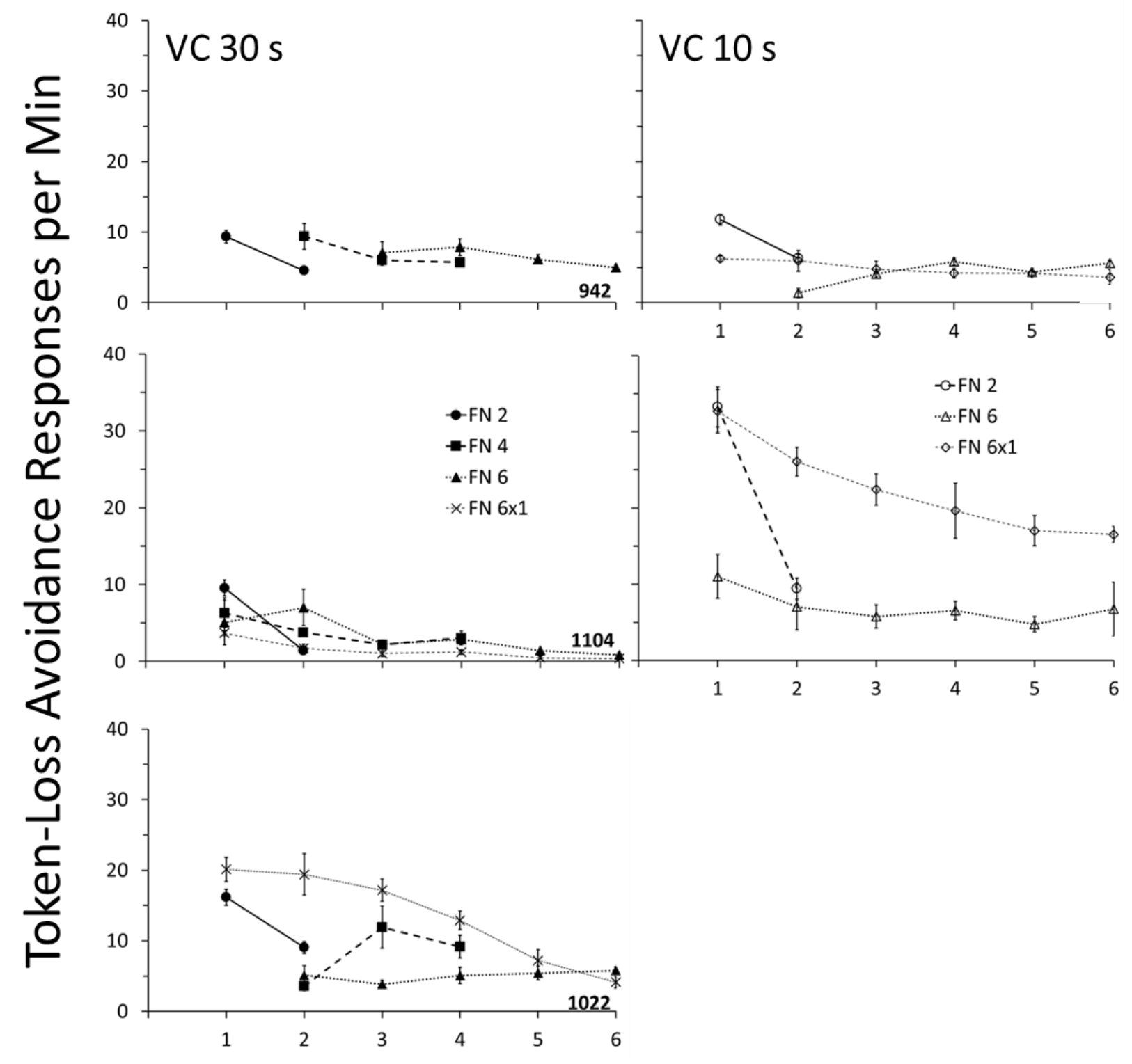

Tokens Present

Figure 12. Aggregate local response rates on the avoidance key as a function of the number of tokens present in the last 5 sessions of each condition in Experiment 2. A VC 30-s and VC 10-s schedule of token-loss frequency was in effect for graphs in the left and right columns, respectively. Data points represent responding between token delivery and the beginning of the exchange period. Error bars represent \pm 1 standard error. 


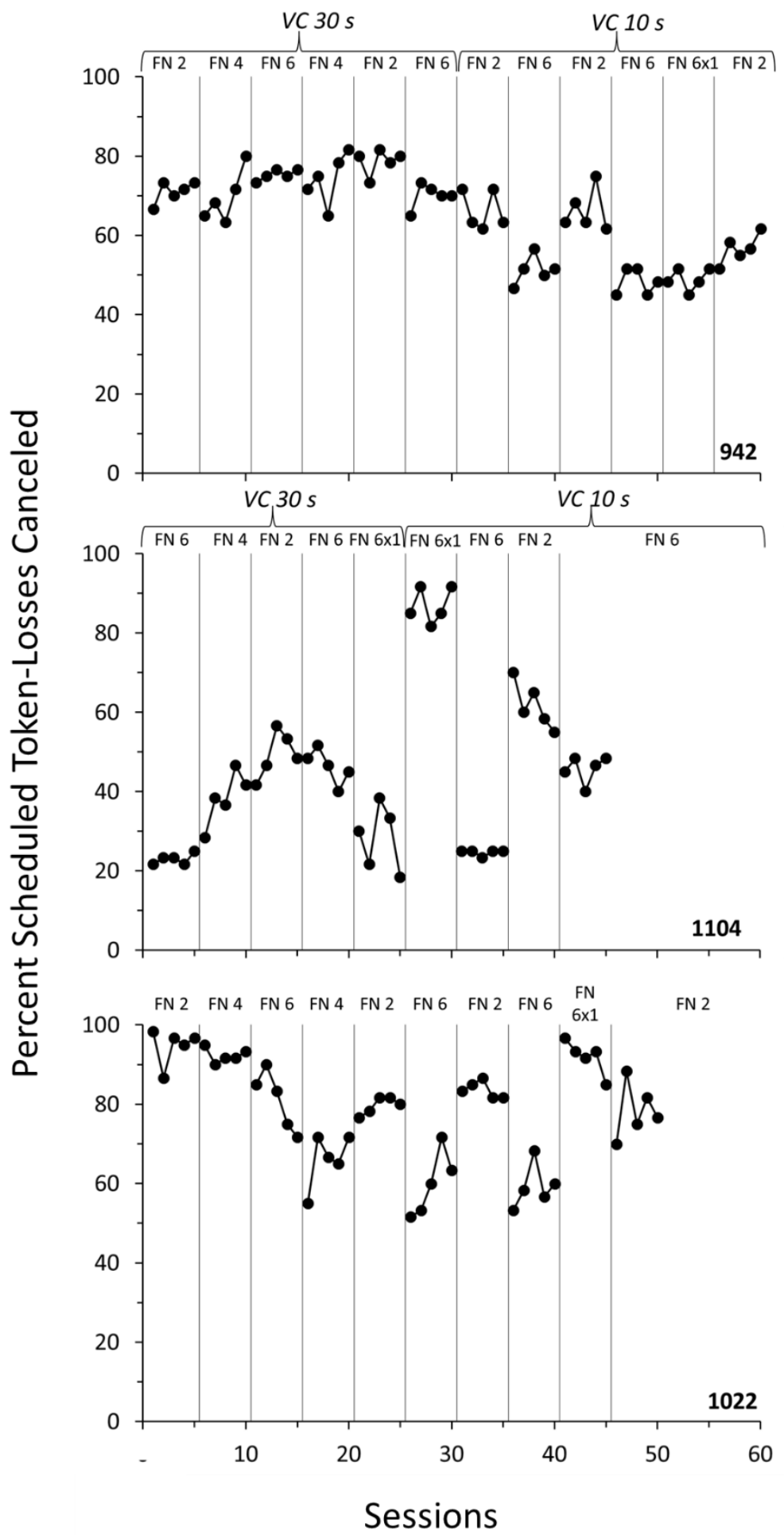

Figure 13. Percent of scheduled token-losses canceled across the last 5 sessions in each condition of Experiment 2. Labels indicate schedule of token-loss frequency of the number of tokens delivered at the beginning of each token-loss avoidance period. 


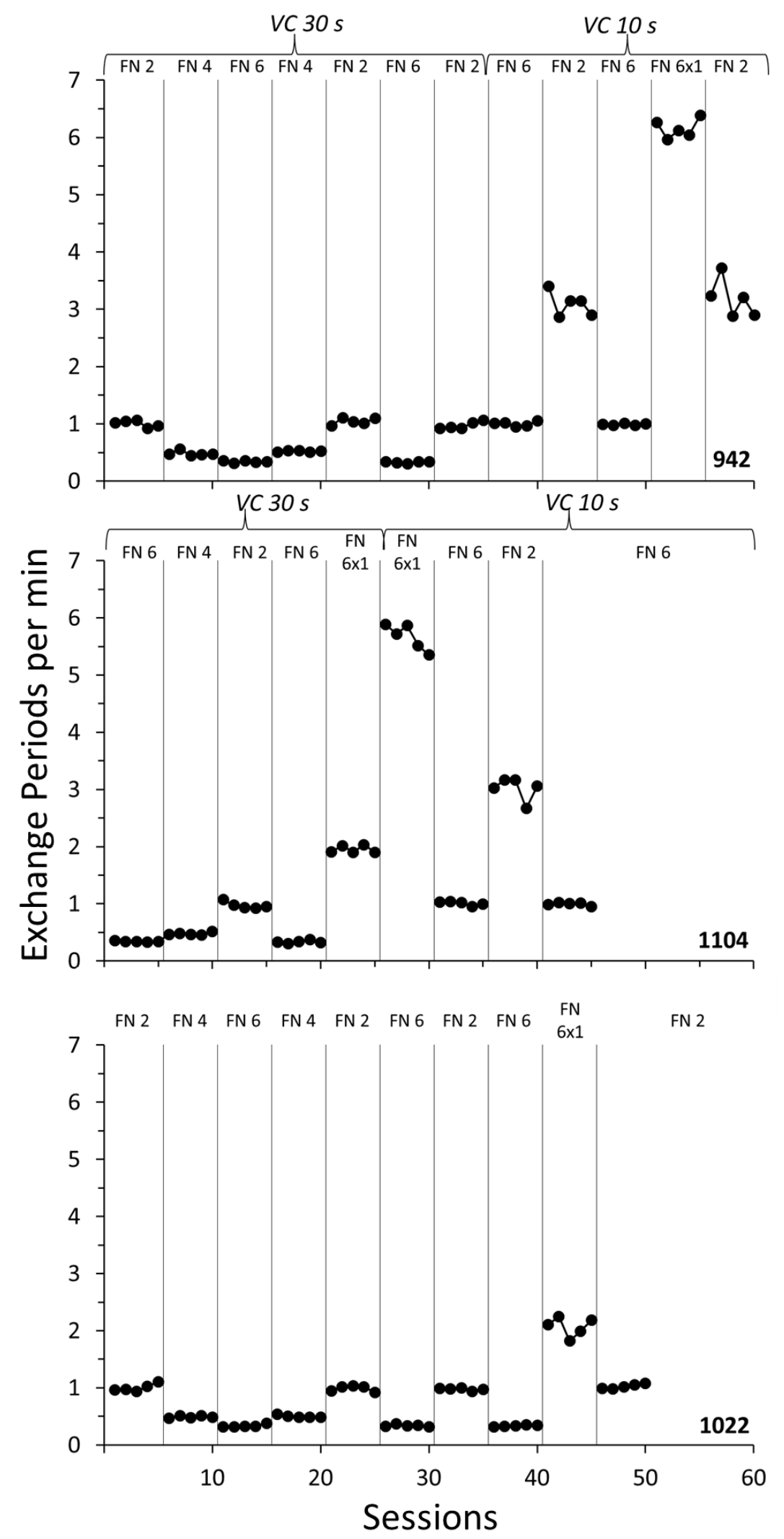

Figure 14. Rate of exchange periods across conditions of Experiment 2. 


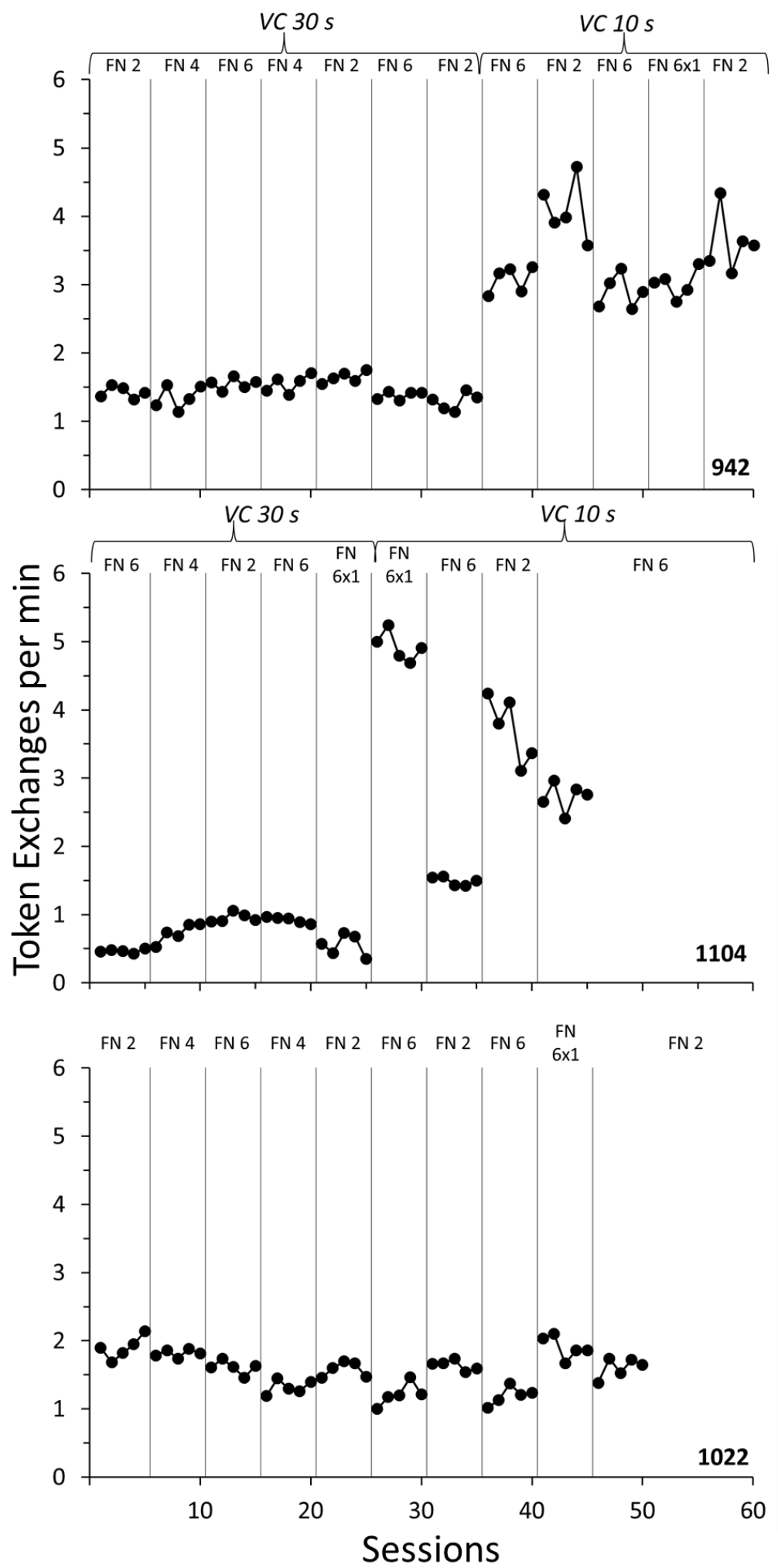

Figure 15. Rate of food deliveries across conditions of Experiment 2. Each token exchanged resulted in one 2.5-s access to food. 


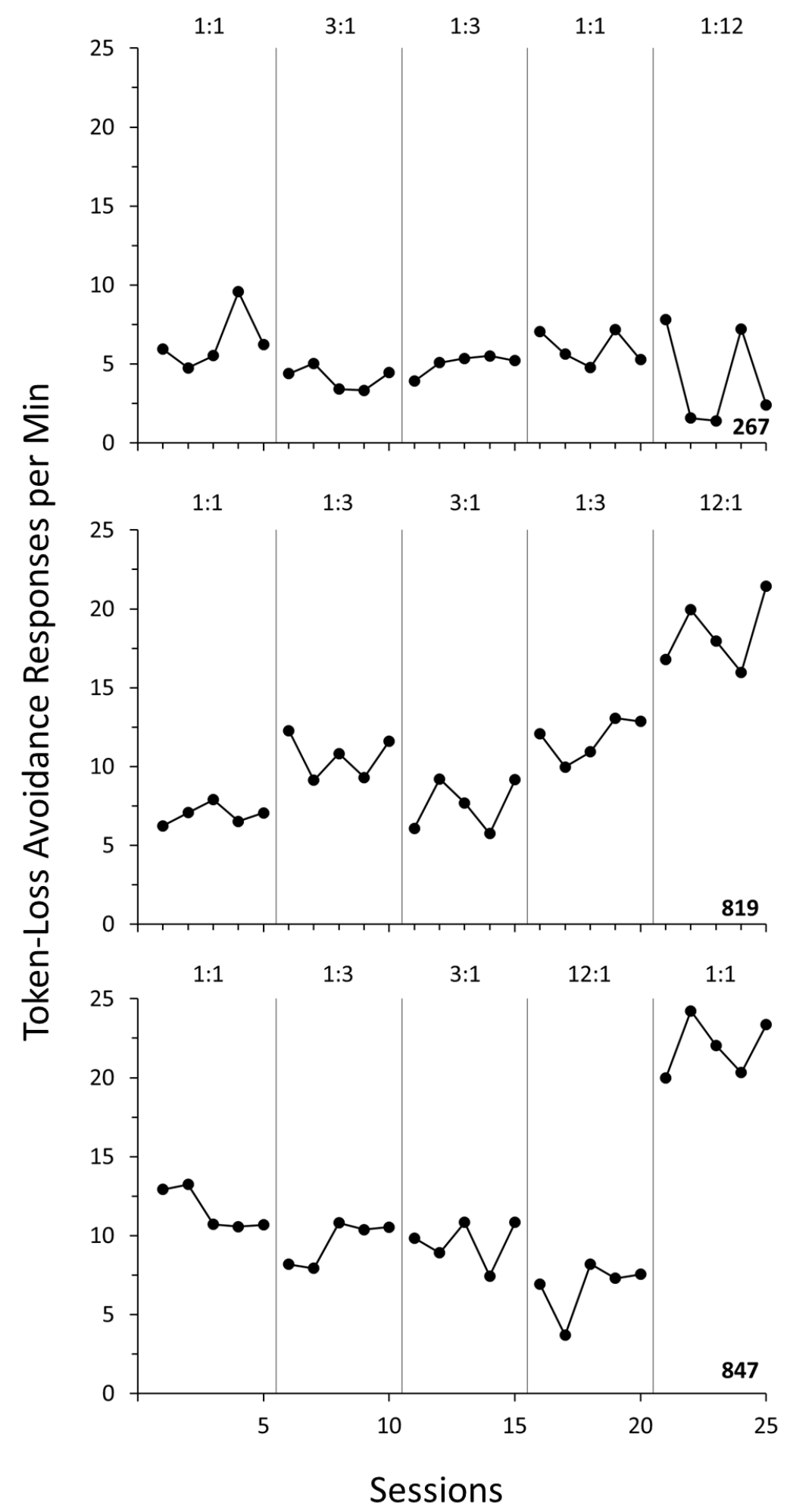

Figure 16. Overall response rates across the last 5 sessions of each condition in Experiment 3. Labels indicate the ratio of tokens to food involved in each token exchange. 


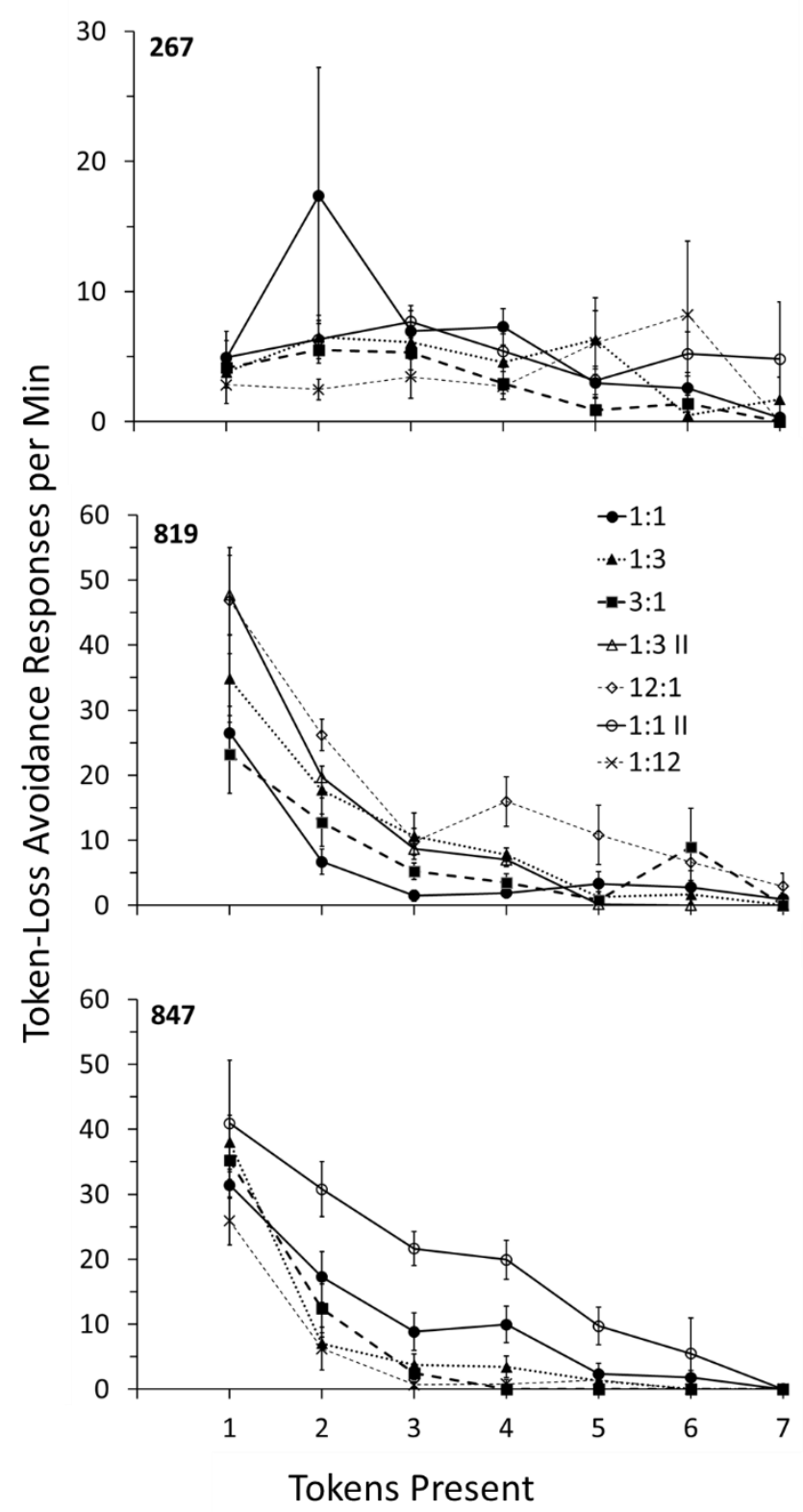

Figure 17. Local response rates on the avoidance key as a function of the number of tokens present in the last 5 sessions of each condition of Experiment 3. Data points represent responding between token delivery and the removal of a single token or the beginning of the exchange period, whichever occurs first. Error bars represent \pm 1 standard error. The y-axis for Pigeon 267 is scaled differently. 


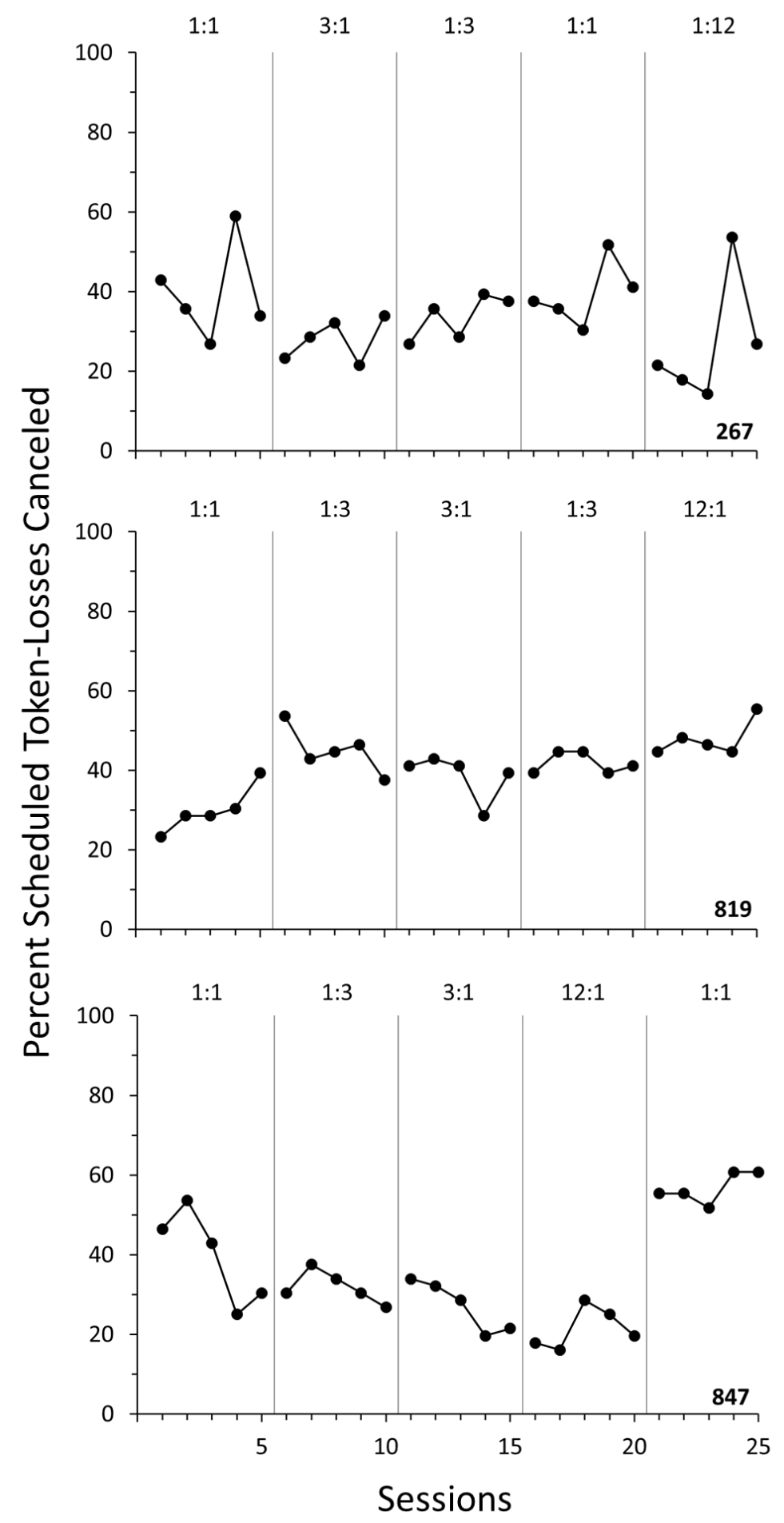

Figure 18. Percent of scheduled token-losses canceled across the last 5 sessions in each condition of Experiment 3. Labels indicate the token-exchange schedule as a ratio of tokens to food. 


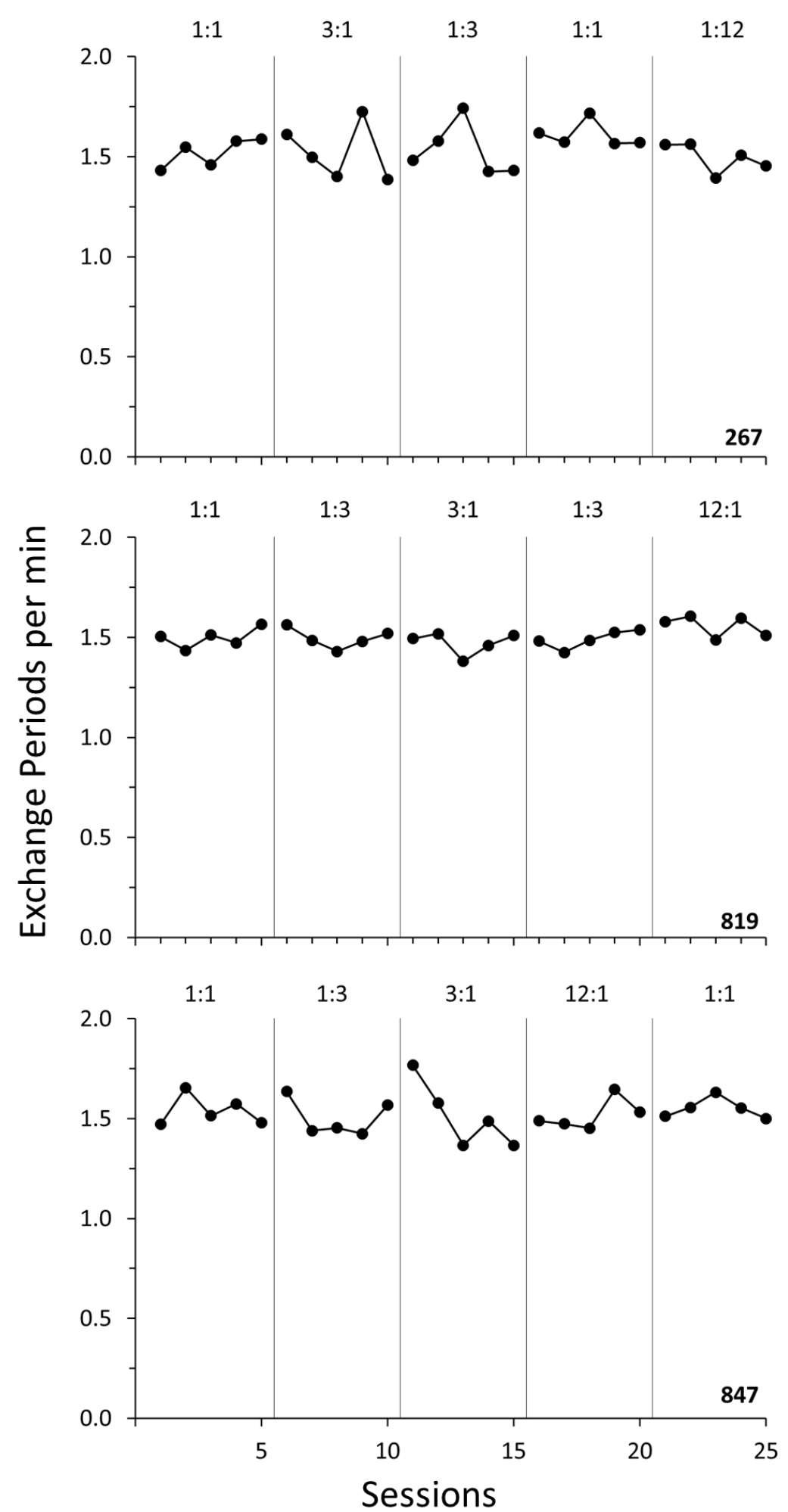

Figure 19. Rate of exchange periods across conditions in Experiment 3. Labels indicate the token-exchange schedule as a ratio of tokens to food. 

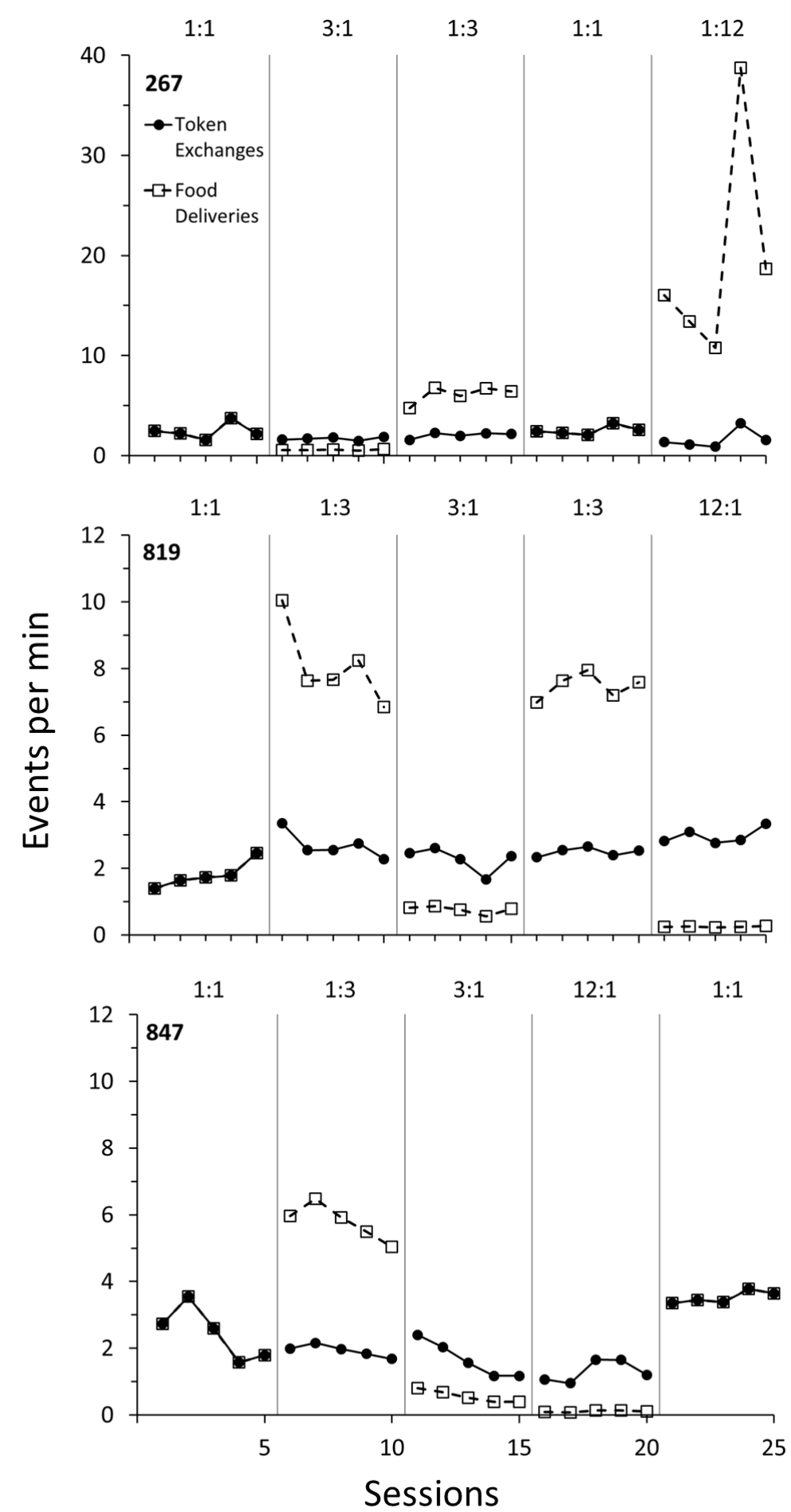

Figure 20. Rate of food deliveries (filled circles) and token exchanges (open squares) across conditions in Experiment 3. Labels indicate the token-exchange schedule as a ratio of tokens to food. The y-axis for Pigeon 267 is scaled differently. 


\section{Appendix A: Reinstatement of Responding in a Token-Loss Avoidance Arrangement}

Reinstatement involves the recurrence of a previously extinguished response following the response-independent delivery of a reinforcer or stimuli associated with reinforcer delivery (Franks \& Lattal, 1976; Shaham, Shalev, Lu, Wit, \& Stewart 2003; Thrailkill, \& Shahan, 2012). Following the EXT condition of Experiment 1, response rates were at or near zero for all three pigeons. Rather than immediately begin the undertaking of the extensive pretraining required with pigeons naïve to the token-loss avoidance arrangement, it was seen if responding previously maintained in a token-loss avoidance arrangement could be reinstated.

\section{Method}

Pigeons 267, 819, and 847 from Experiment 1 served as subjects. Sessions were conducted in the same operant chamber described in Experiment 1. Sessions were conducted in the same manner as described in Experiment 1 except as noted below.

Extinction. The last five sessions of the EXT condition from Experiment 1 were used as a baseline against which to compare the effects of subsequent conditions.

Token Reinstatement. This condition lasted for 5 sessions. Sessions operated similarly to the EXT condition except that a predetermined number of token-losses were canceled in each session. Which token-losses were canceled was determined by first creating an array of 56 zeroes and ones. The number of ones in the array was equal to the average number of token-losses canceled in the last five sessions of the second VC 10-s condition from Experiment 1, rounded to the nearest integer. These numbers were 22, 27, and 31 token-losses canceled for Pigeons 267, 819 , and 847, respectively. At the end of each cycle in session, a zero or one would be drawn from the array randomly without replacement. If a one was drawn, the scheduled token-loss was canceled. Responding on the avoidance key was recorded but had no programmed effect. 
If any tokens remained when an exchange period began, responses on the exchange key turned off the right-most token, the houselight, and exchange key for $2.5 \mathrm{~s}$. The hopper was not raised, and no food was presented. Exchange periods ended when all remaining tokens were exchanged or 30 s elapsed, whichever came first.

Token+Food Reinstatement. This condition lasted for 5 sessions. Sessions operated as described in the Token Reinstatement condition except that when a token was exchanged, the hopper was raised and food was presented.

VC 10 s. Sessions operated as described in the VC 10-s conditions in Experiment 1. These sessions served as the first 5 sessions of Experiment 3.

\section{Results and Discussion}

Figure A1 shows overall response rates across conditions. Responding for all pigeons was at or near zero levels across the last five sessions of EXT. In the Token Reinstatement condition, response rates increased for all pigeons when scheduled token-losses were canceled response-independently and could be exchanged for the stimuli associated with food delivery. For Pigeon 267, the increase in responding occurred in the first session followed by a rapid decline back to zero. For Pigeon 819, responding recurred across the first four sessions before dropping back to zero. For Pigeon 847, response rates increased above those in the EXT condition in the fourth session. Overall response rates increased for all pigeons in the Token+Food Reinstatement condition. Response rates increased further for Pigeons 267 and 819 when the VC 10-s schedule of token-loss avoidance was reimplemented.

Figure A2 shows response rates as a function of the number of tokens present across conditions. Data points were determined in the same manner as in Experiment 1. For all pigeons, response rates were lowest in the EXT condition. For Pigeons 819 and 847, higher rates of 
responding occurred when fewer tokens were present in the other conditions. This pattern of responding did not occur for Pigeon 267. When scheduled token-losses were canceled responseindependently and could be exchanged for the stimuli associated with food delivery, patterns of responding recurred that were similar to those that occurred when scheduled token-losses were canceled response-dependently.

Figure A3 shows the percentage of scheduled token-losses canceled across conditions. Zero scheduled token-losses were canceled in the EXT condition. The percentage of scheduled token-losses canceled in the Token Reinstatement and Token+Food Reinstatement conditions differed between subjects but were the same within subjects. In the VC 10-s condition, the percentage of scheduled token-losses canceled declined for Pigeons 819 and 847 and increased overall for Pigeon 267. Figure A4 shows there was no change in exchange period frequency across conditions.

Figure A5 shows the rate of token exchanges across conditions. In each condition except the Token Reinstatement condition, each token exchange resulted in the delivery of food. Zero token exchanges occurred in the EXT condition. Token exchanges declined across sessions for all pigeons in the Token Reinstatement condition as food was never delivered in this condition. The rate of token exchanges recovered in the Token+Food Reinstatement condition. In the VC 10-s condition, the rate of token exchanges declined overall for Pigeons 819 and 847 and increased overall for Pigeon 267.

Despite declining rates of token exchanges in the VC 10-s condition (Figures A4 and A5), response rates increased or remained the same compared to those in the Token+Food Reinstatement condition (Fig. 1). Imposing the operant contingency of the VC schedule 
maintained or increased overall response rates relative to the Token+Food Reinstatement condition.

Tokens and stimuli associated with their exchange may have had discriminative or reinforcing properties (Bullock \& Hackenberg, 2015; Shahan, 2010). These properties were sufficient to reinstate responding previously maintained in a token-loss avoidance arrangement. Bullock and Hackenberg (2015) state that tokens have multiple functions: as eliciting stimuli, discriminative stimuli, and as conditioned reinforcers. They also state that these functions depend on the contingencies in which they are embedded. In this case, when token-losses were canceled response-independently, response rates that varied as a function of the number of tokens present were reinstated. The tokens and the stimuli that accompanied their exchange likely had discriminative properties that occasioned these patterns of responding. When the operant contingency of the VC 10-s schedule was reimplemented, those patterns persisted and response rates increased or maintained despite decreasing rates of primary reinforcement. The responsedependent avoidance of token-loss served to increase responding, likely because of a reinforcing function of avoidance of token-loss.

These results may invite further investigation into reinstatement and recurrence more generally as it pertains to responding maintained by conditioned reinforcers (Thrailkill \& Shahan, 2012) and negative reinforcement (Bruzek, Thompson, \& Peters, 2009; Li et al., 2007). 


\section{References}

Bruzek, J. L., Thompson, R. H., \& Peters, L. C. (2009). Resurgence of infant caregiving responses. Journal of Experimental Analysis of Behavior, 92, 327-343.

Bullock, C. E., \& Hackenberg, T. D. (2015). The several roles of stimuli in token reinforcement. Journal of the Experimental Analysis of Behavior, 103, 269-287.

Franks, G. J., \& Lattal, K. A. (1976). Antecedent reinforcement schedule training and operant response reinstatement in rats. Animal Learning \& Behavior, 4(4), 374-378.

Li, Y., Liu, X., Chen, H., Deng, H., Xiang, X., Chen, H., \& Hao, W. (2007). Development, extinction, and reinstatement of morphine withdrawal-induced conditioned place aversion in rats. Addiction Biology, 12, 470-477.

Shaham, Y., Shalev, U., Lu, L., de Wit, H., \& Stewart, J. (2003). The reinstatement model of drug relapse: History, methodology, and major findings. Psychopharmacology, 168(1-2), $3-20$.

Shahan, T. A. (2010). Conditioned reinforcement and response strength. Journal of the Experimental Analysis of Behavior, 93, 269-289.

Thrailkill, E. A., \& Shahan, T. A. (2012). Resistance to change and relapse of observing. Journal of the Experimental Analysis of Behavior, 97, 281-304. 


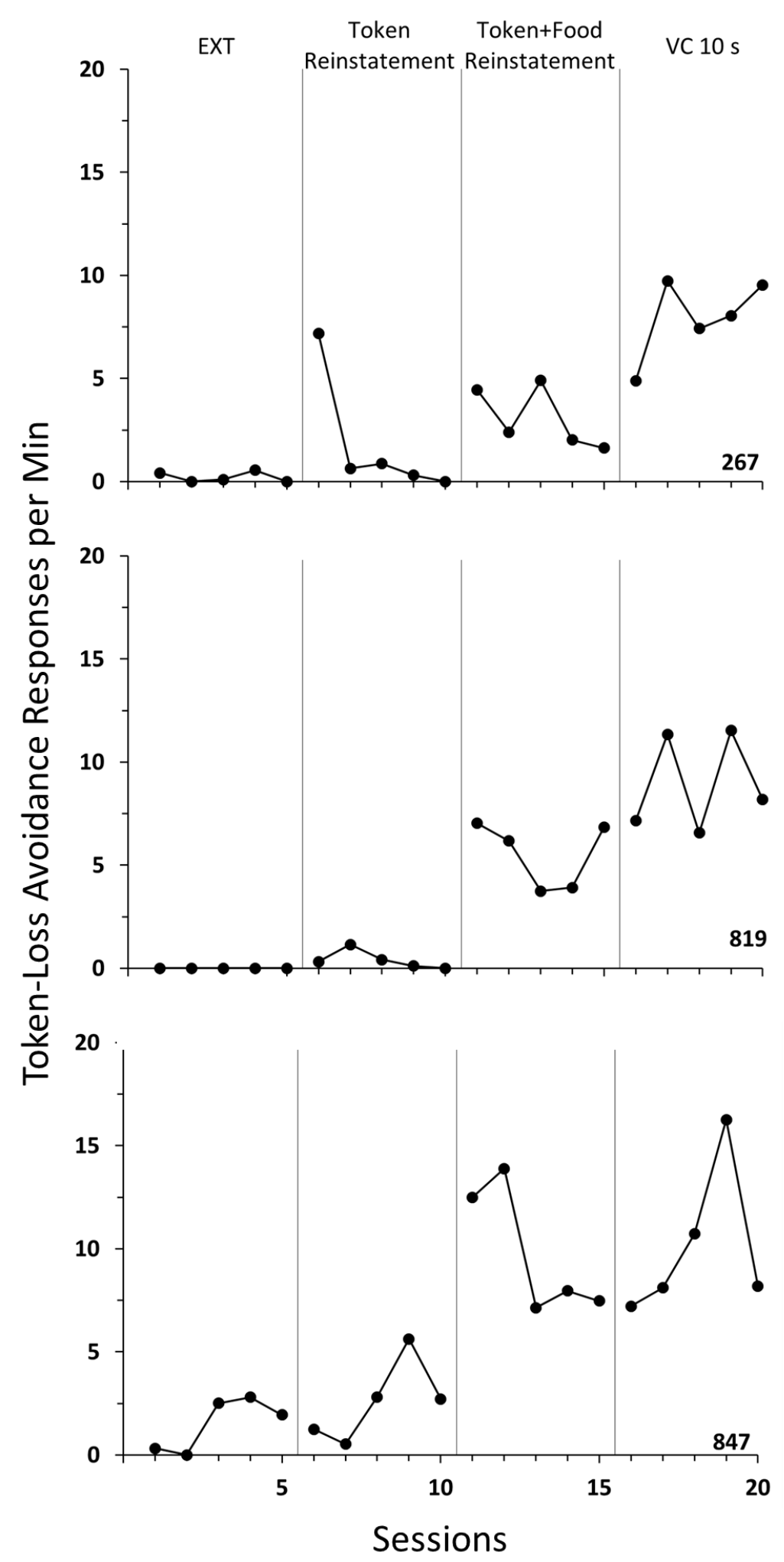

Figure A1. Overall response rates on the avoidance key during token-loss avoidance periods across conditions. Labels indicate the condition in effect. 


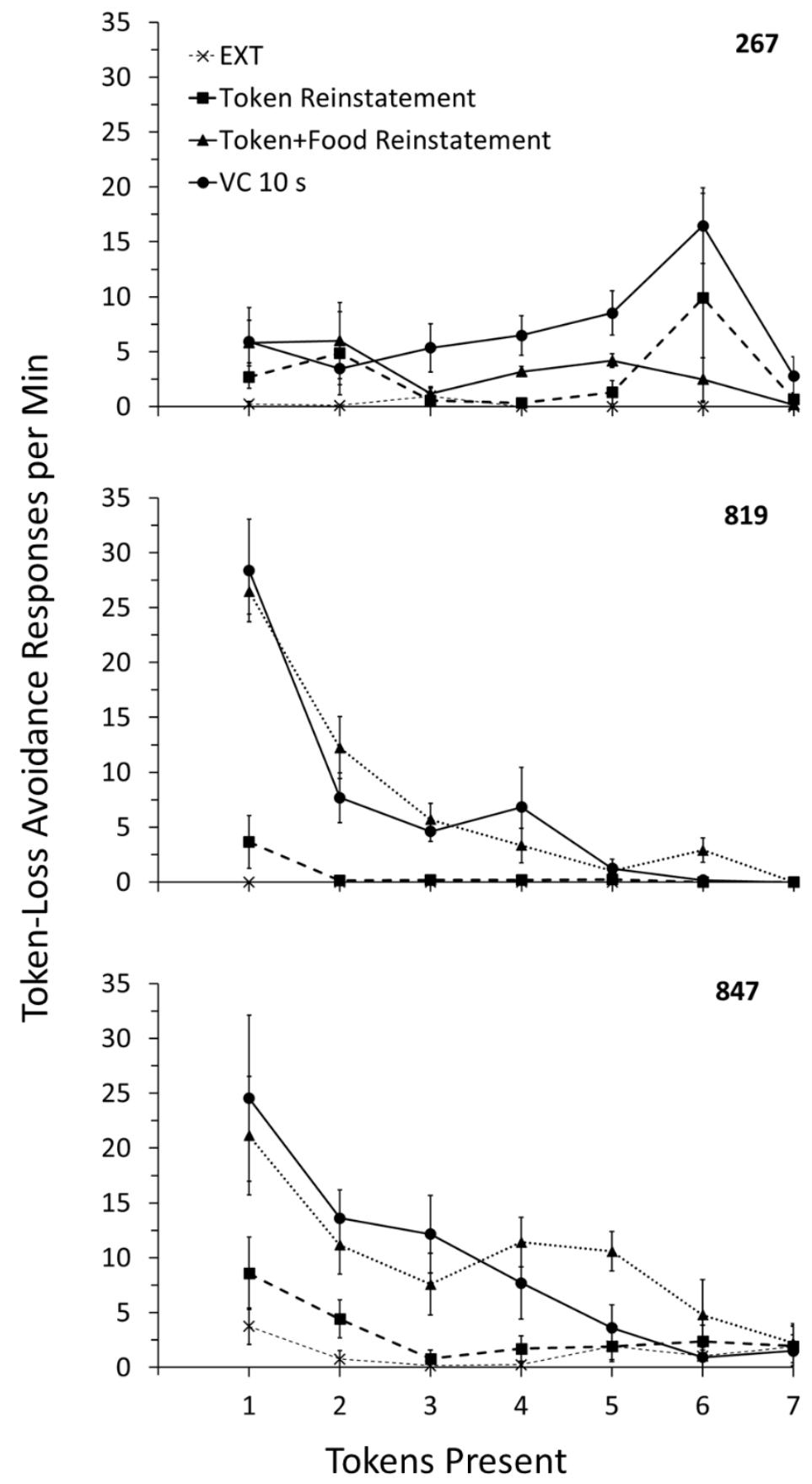

Figure A2. Local response rates on the avoidance key as a function of the number of tokens present in the last 5 sessions of each condition of Experiment 1. Data points represent responding between token delivery and the removal of a single token or the beginning of the exchange period, whichever occurs first. Error bars represent \pm 1 standard error. 


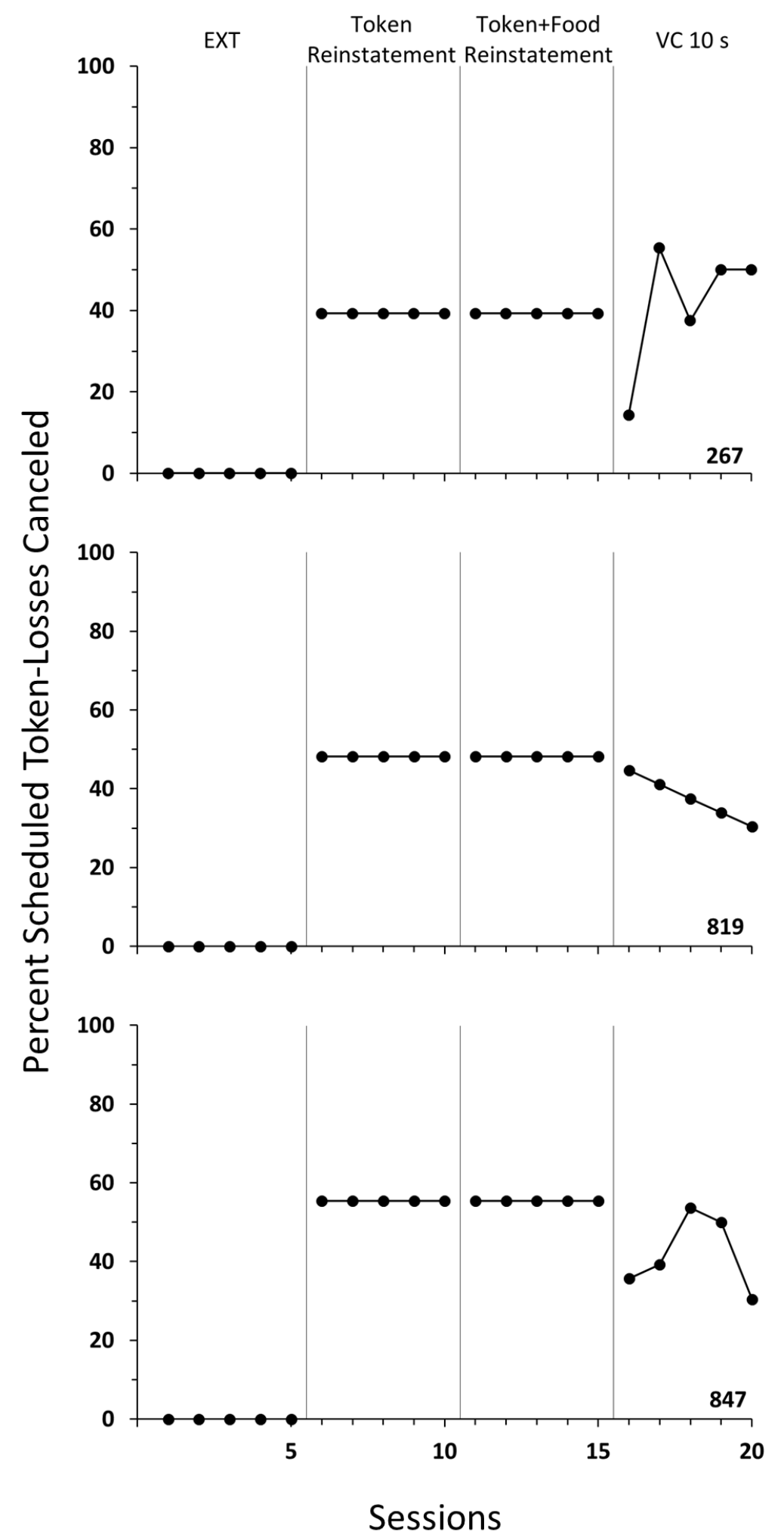

Figure A3. Percent of scheduled token-losses canceled in each condition. Labels indicate the condition in effect. 

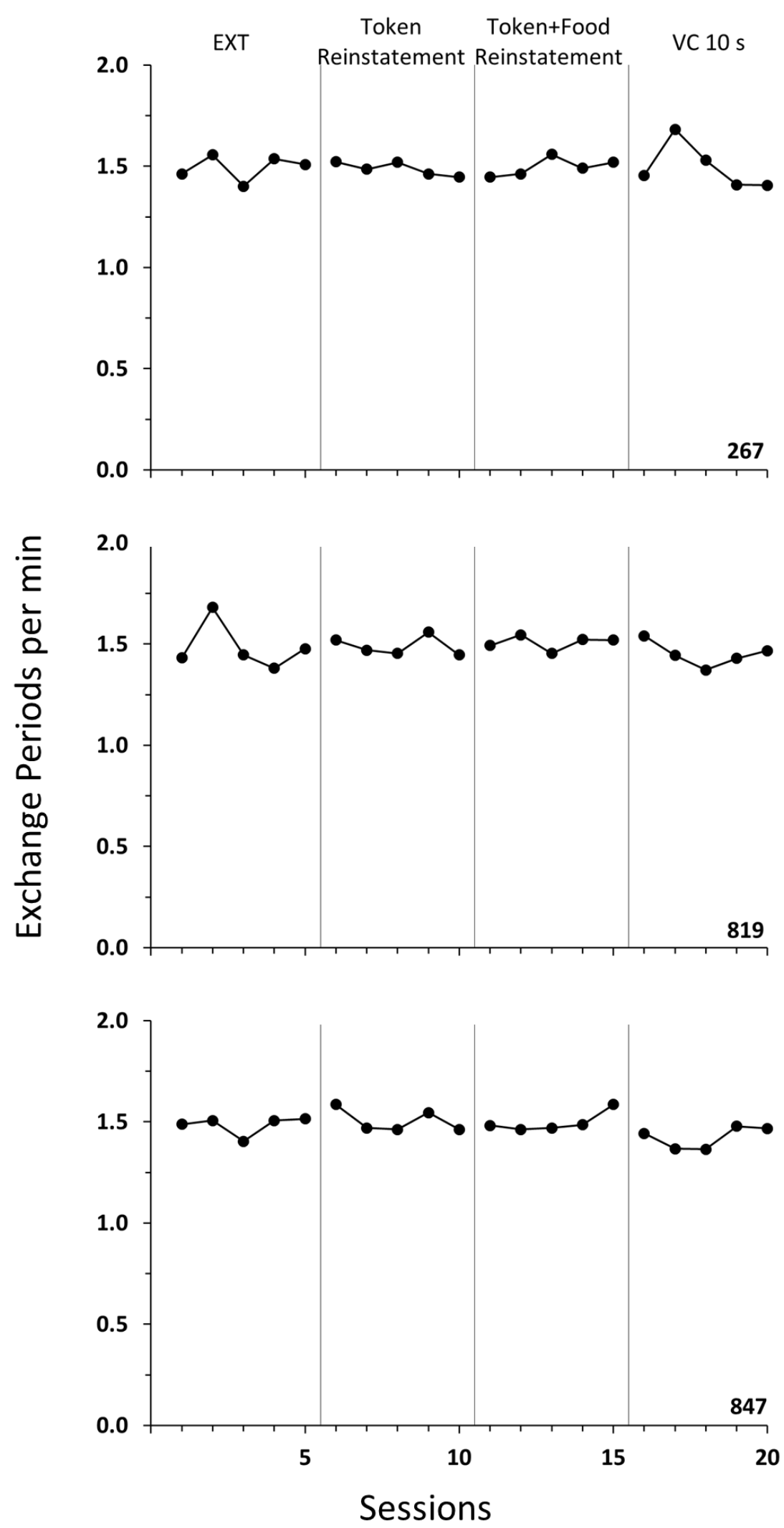

Figure A4. Rate of exchange periods across conditions. Labels indicate the condition in effect. 

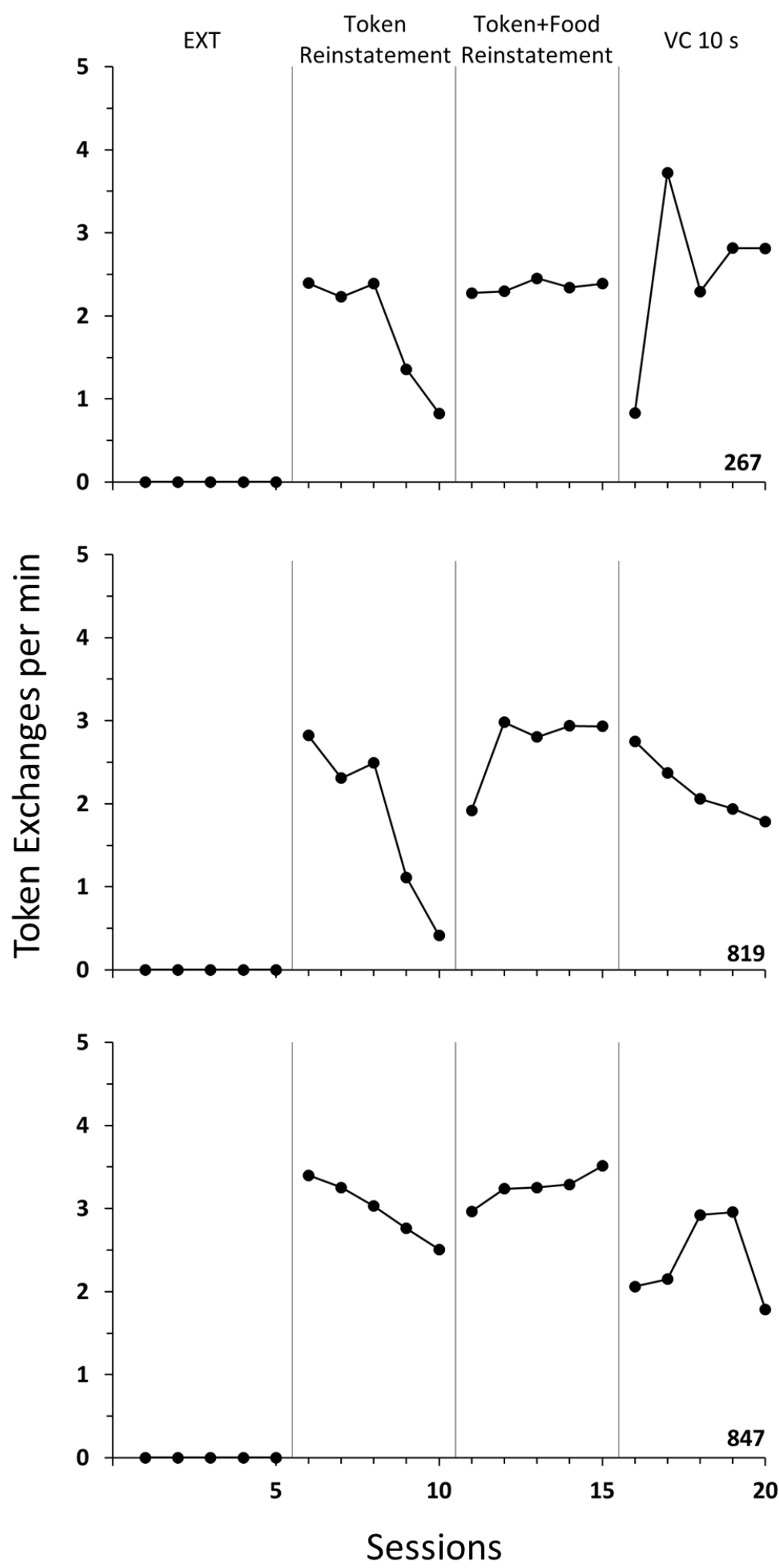

Figure A5. Rate of token exchanges across conditions. Each token exchanged resulted in one 2.5-s access to food except for in the Token Reinstatement condition. 


\section{Appendix B: Responding on a Multiple Schedule of Token-Loss Avoidance and Token- Production}

A symmetrical law of effect supposes that equivalent gains and losses should have equivalent effects on behavior. Under this assumption, an organism ought to respond equally as much to avoid a loss as it would to produce an equivalent gain. Loss-aversion (Kahnemann \& Tversky, 1979, 2000) supposes that losses exert greater control over behavior than do equivalent gains. Under this assumption, an organism ought to respond more to avoid a loss than it would to produce an equivalent gain. In the operant literature, the results of some experiments support a symmetrical law of effect (Iwata \& Bailey, 1974; Magoon \& Critchfield, 2008) and others support loss-aversion (Donaldson, DeLeon, Fisher, \& Kahng, 2014; Rasmussen \& Newland, 2008).

Following Experiment 2, Pigeon 1022 was placed on a multiple schedule. In one component, responding resulted in token-loss avoidance. In the other component, responding resulted in token production on an equivalent schedule. This was done as pilot work in assessing whether equivalent schedules of token production or token-loss avoidance would maintain equivalent levels of responding.

\section{Method}

Pigeons 1022 from Experiment 2 served as the only subject. Sessions were conducted in the same operant chamber described in Experiment 1. Sessions were conducted in the same manner as described in Experiment 1 except as noted below.

A two-component multiple schedule operated in each session. One component (Avoid) operated as the VC 10-s token-loss avoidance condition described in Experiment 1. In the other component (Production), a VC 10-s token-production schedule operated. In this component, the 
token-production period began with no tokens illuminated. A response on the right (production) key during each VC 10-s cycle resulted in the left-most token turning on at the end of the cycle. If no response occurred during the VC 10-s cycle, no change occurred at the end of the cycle. The number of cycles in each token-production period of the Production component was determined by randomly selecting without replacement from an array consisting of the numbers 1-7. For example, if the number 1 was selected, one VC 10-s cycles would elapse during the token-production period. If the number 2 was selected, two VC 10-s cycles would elapse during the token-production period. After the selected number of cycles elapsed, the production key turned off and the center (exchange) key was illuminated green. This began the exchange period. Exchange periods operated in the same manner described in Experiment 1 for both components. Whatever tokens were produced during the token-production period could be exchanged for 2.5s access to food.

Each component continued until the first exchange period ended following $28 \mathrm{VC} 10-\mathrm{s}$ cycles. A maximum of 28 tokens could be exchanged in each component. Each component occurred twice in each session. The first component was randomly determined. Each component occurred once before either occurred twice. The left key was illuminated red during the tokenloss avoidance period of the Avoid component and the center and right keys were dark. The right key was illuminated white during the token-production period of the Production component and the left and center keys were dark. Responses on dark keys had no programmed effect.

A minimum of 15 sessions were conducted until responding on both responding in both components appeared stable as determined by visual analysis. 


\section{Results and Discussion}

Fifteen sessions were conducted in this pilot study. Table B1 shows the average frequency of exchange periods, food exchanges, and the percentage of available tokens exchanged in both components across the last 5 sessions. Exchange periods occurred with about the same frequency in both components. The average rate of food deliveries was greater in the Avoid component. The average percentage of available tokens exchanged represents the percentage of scheduled token-losses canceled in the Avoid component and the percentage of available tokens produced in the Production component. Fifty-six tokens total could be exchanged in each component if all token-losses were canceled in the Avoid component and all available tokens were produced in the Production component. A greater percentage of the 56 possible tokens were exchanged in the Avoid component than in the Production component. The greater rate of food deliveries and percentage of token exchanges may have affected the differences in responding described below.

Figure B1 shows response rates in the Avoid and Production components across the last 5 sessions. Generally higher rates of responding occurred in the Avoid component, but response rates converged in the last session. Greater rates of responding in the Avoid component would support token-loss avoidance having functioned as a more potent reinforcer than tokenproduction on an equivalent schedule. The higher rates of responding shown in Figure B1 would seem to support loss-aversion over a symmetrical law of effect, but further investigation is warranted. It is possible that Pigeon 1022's longer history of responding on the key associated with the Avoid component and this key's position in the box may have contributed to a bias in responding on the this key. Future conditions will involve switching the contingencies associated 
with each key to evaluate whether key color or position has any influence on responding in these components.

Figure B2 shows average response rates in the Avoid and Production components across the last 5 sessions as a function of the number of tokens present. In the Avoid component, generally higher levels of responding occurred when fewer tokens were present except for a slightly higher response rate when 7 tokens were present. These results are similar to those obtained in Experiments 1-3. In the Production component, a high response rate occurred when 0 tokens were present. Lower response rates occurred when 1 or 2 tokens were present followed by increasing response rates as more tokens were present. In both components, response rates tended to be higher when closer in time to the exchange period. This pattern of responding is generally similar to that produced in other research that has examined changes in responding maintained by token production (Bullock \& Hackenberg, 2006, 2015; Foster, Hackenberg, \& Vaidya, 2001; Malagodi, Webbe, \& Waddell, 1975). Additionally, the patterns of responding that occurred as a function of the number of tokens present in the Avoid and Production components were generally symmetrical. This provides further support that the tokens were providing similar discriminative functions in both components.

Further research should be conducted along this line comparing responding maintained by token-loss avoidance and the production of tokens. Further investigation may provide better understanding about the symmetrical law of effect and loss-aversion as basic behavioral processes. 


\section{References}

Bullock, C. E., \& Hackenberg, T. D. (2006). Second-order schedules of token reinforcement with pigeons: Implications for unit price. Journal of the Experimental Analysis of Behavior, 85, 95-106.

Bullock, C. E., \& Hackenberg, T. D. (2015). The several roles of stimuli in token reinforcement. Journal of the Experimental Analysis of Behavior, 103, 269-287.

Donaldson, J. M., DeLeon, I. G., Fisher, A. B., \& Kahng, S. (2014). Effects of and preference for conditions of token earn versus token loss. Journal of Applied Behavior Analysis, 47, 537-548.

Foster, T. A., Hackenberg, T. D., \& Vaidya, M. (2001). Second-order schedules of token reinforcement with pigeons: Effects of fixed- and variable-ratio exchange schedules. Journal of the Experimental Analysis of Behavior, 76, 159-178.

Iwata, B. A., \& Bailey, J. S. (1974). Reward versus cost token systems: An analysis of the effects on students and teacher. Journal of Applied Behavior Analysis, 7, 567-576.

Kahneman, D., \& Tversky, A. (1979). Prospect theory: An analysis of decisions under risk. Econometrica, 47, 263-291.

Kahneman, D., \& Tversky, A. (Eds.) (2000). Choices, values, and frames. New York: Cambridge University Press.

Magoon, M. A., \& Critchfield, T. S. (2008). Concurrent schedules of positive and negative reinforcement: Differential-impact and differential-outcomes hypotheses. Journal of the Experimental Analysis of Behavior, 90, 1-22. 
Malagodi, E. F., Webbe, F. M., \& Waddell, T. R. (1975). Second-order schedules of token reinforcement: Effects of varying the schedule of food presentation. Journal of the Experimental Analysis of Behavior, 24, 173-181.

Rasmussen, E. B., \& Newland, M. C. (2008). Asymmetry of reinforcement and punishment in human choice. Journal of the Experimental Analysis of Behavior, 89, 157-167. 
Table B1

Average Frequency of Exchange Periods and Food Deliveries and the Percentage of Available Tokens Exchanged in the Avoidance and Production Components across the Last 5 Sessions

Dependent Variable Avoid Production

Percent of Available Tokens Exchanged

55.71

Exchange Periods per min

1.48

1.55

Token Exchanges per min 


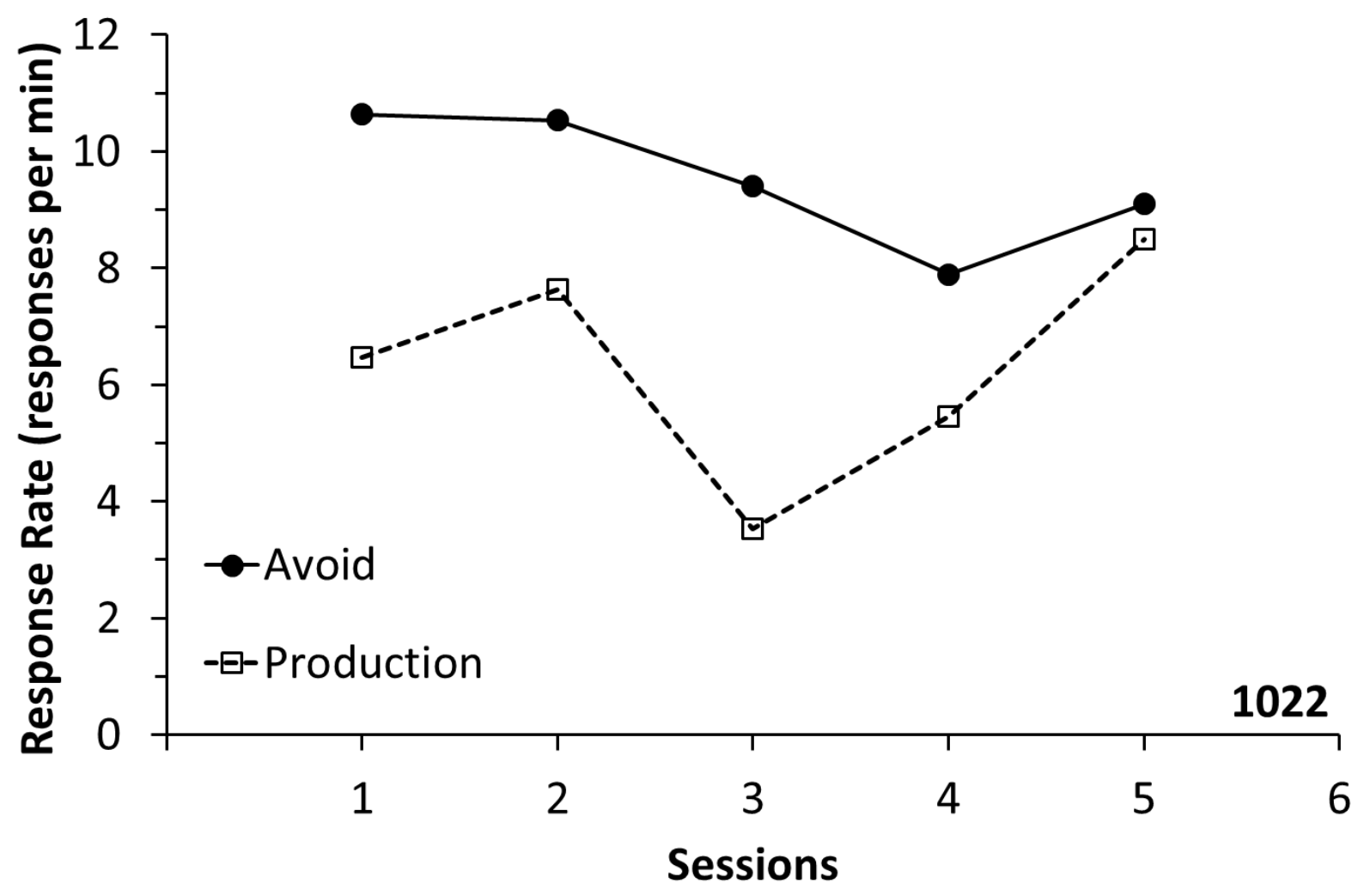

Figure B1. Overall response rates in the Avoid and Production components across the last 5 sessions. 


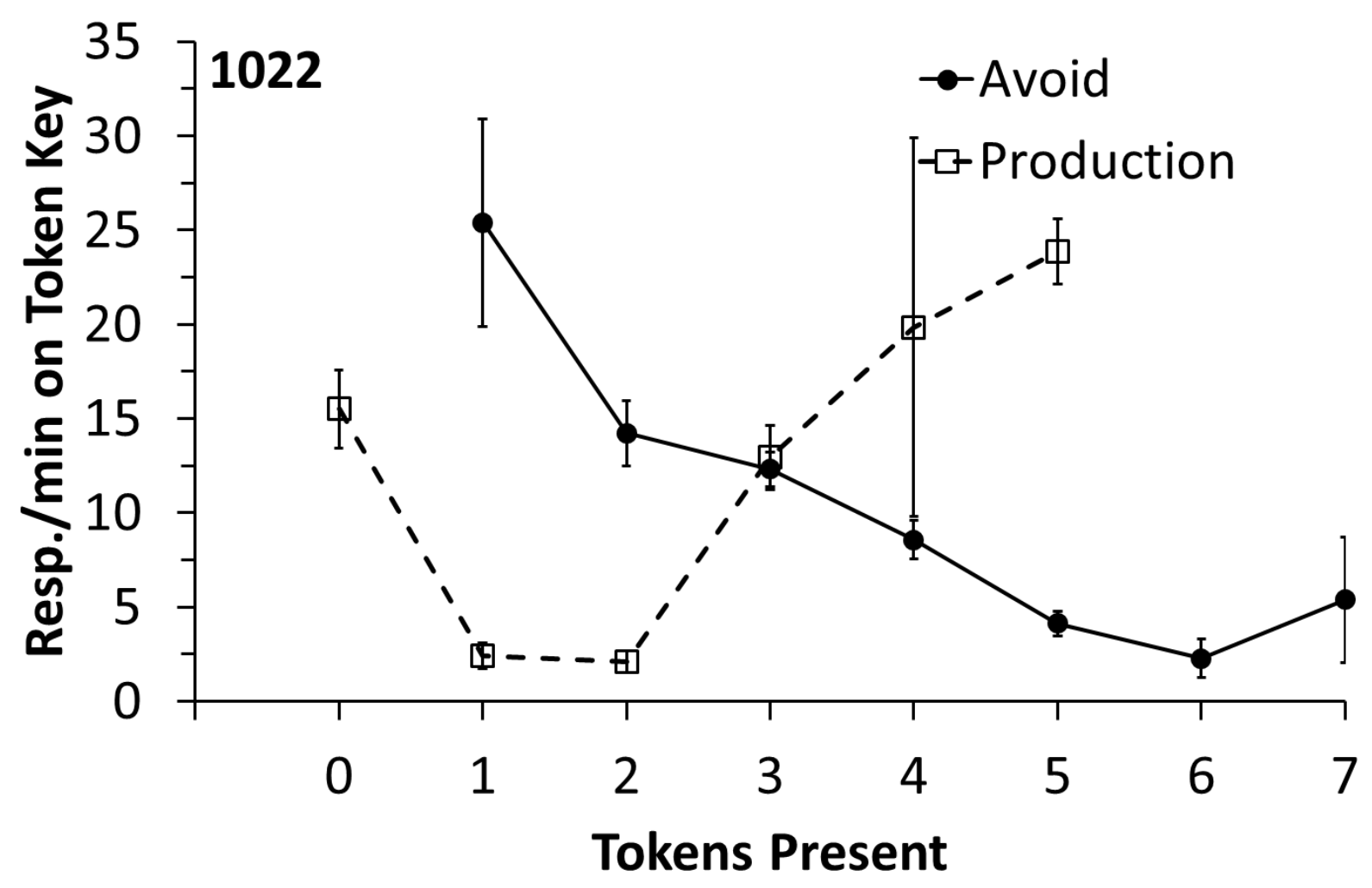

Figure B2. Local response rates in the Avoid and Production components across the last 5 sessions as a function of the number of tokens present. Error bars represent \pm 1 standard error. 